NPAC-08-19

MADPH-08-1522

\title{
Leptoquarks and Neutrino Masses at the LHC
}

\author{
Pavel Fileviez Pérez ${ }^{1}$, Tao Han ${ }^{1}$, Tong $\mathrm{Li}^{1,2}$, Michael J. Ramsey-Musolf ${ }^{1,3}$ \\ ${ }^{1}$ Department of Physics, University of Wisconsin, Madison, WI 53706, USA \\ ${ }^{2}$ Department of Physics, Nankai University, Tianjin 300071, P.R. China \\ ${ }^{3}$ Kellogg Radiation Laboratory, California Institute of Technology, Pasadena, CA 91125 USA
}

(Dated: February 20, 2013)

\begin{abstract}
The properties of light leptoquarks predicted in the context of a simple grand unified theory and their observability at the LHC are investigated. The $S U(5)$ symmetry of the theory implies that the leptoquark couplings to matter are related to the neutrino mass matrix. We study the resulting connection between neutrino masses and mixing parameters and the leptoquark decays, and show that different light neutrino hierarchies imply distinctive leptoquark decay signatures. We also discuss low-energy constraints implied by searches for charged lepton flavour violation, studies of meson decays, and electroweak precision data. We perform a detailed parton-level study of the leptoquark signals and the Standard Model backgrounds at the LHC. With the clean final states containing a dilepton plus two jets, the QCD production of the leptoquark pair can be observed for a leptoquark mass of one $\mathrm{TeV}$ and beyond. By examining the lepton flavor structure of the observed events, one could further test the model predictions related to the neutrino mass spectrum. In particular, $b$-flavor tagging will be useful in distinguishing the neutrino mass pattern and possibly probing an unknown Majorana phase in the Inverted Hierarchy or the Quasi-Degenerate scenario. Electroweak associated production of the leptoquark doublet can also be useful in identifying the quantum numbers of the leptoquarks and distinguishing between the neutrino mass spectra, even though the corresponding event rates are smaller than for QCD production. We find that with only the clean channel of $\mu+E_{T}+$ jets, one could expect an observable signal for a leptoquark masses of about $600 \mathrm{GeV}$ or higher.
\end{abstract}

\section{INTRODUCTION}

The possibility of explaining all fundamental interactions in nature in a unified framework is one of the main motivations for considering physics beyond the Standard Model (SM) of particle physics. In this respect, Grand Unified Theories (GUTs) are one of the most appealing SM extensions, as they may allow one to understand the origin of the SM interactions and predict both the quantization of the electric charge and the weak mixing angle [1]. In these theories the unification of gauge couplings is realized at a scale 
$M_{G U T} \approx 10^{14-16} \mathrm{GeV}$. The unification of the matter fields implies the decay of the lightest baryon, the proton [2], and the generic existence of scalar and vector leptoquarks (denoted by LQ unless specified otherwise) [3] that carry both lepton- and baryon-numbers, since the quarks and leptons live in the same enlarged representation of the gauge group.

Because of the important role played by LQs in GUTS and other SM extensions as well as their unique phenomenological features, the general properties and phenomenology of LQs have been studied extensively[4, 5]. Recently, a simple GUT based on the SU(5) gauge symmetry that contains light LQs has been proposed in Ref. [6]. In this theory, the Higgs sector is composed of $\mathbf{5}_{\mathbf{H}}, \mathbf{1} \mathbf{5}_{\mathbf{H}}$ and $\mathbf{2} \mathbf{4}_{\mathbf{H}}$; neutrino masses are generated through the Type II seesaw mechanism [7]; and there exist light scalar leptoquarks consistent with the constraints from the gauge coupling unification and experimental lower bounds on the proton lifetime. A distinctive feature of this framework is that the LQ-matter Yukawa interactions are governed by the neutrino mass matrix. Consequently, different scenarios for the light neutrino mass hierarchy lead to broadly distinguishable patterns for LQ interactions with matter. In this paper, we explore this phenomenology in detail, concentrating in particular on its implications for studies at the Large Hadron Collider (LHC).

While several phenomenological and cosmological aspects of this proposal were studied in Refs. [8] and [9], a comprehensive analysis of the corresponding LQ collider phenomenology has not appeared previously in the literature. Here, we report the results of such an analysis. In doing so, we consider the constraints on the model implied by the results of neutrino oscillation experiments, searches for charged lepton flavor violation, and studies of SM-suppressed meson decays. We find that although the constraints from low-energy flavor physics are severe, they do not preclude the possibility of observing a statistically significant LQ signal at the LHC if the LQ mass is sufficiently heavy. The constraints from electroweak precision observables (EWPO) have a marginal impact at best on the LHC discovery potential of the model.

In searching for the LQ at the LHC, we identify the dominant production mechanisms and the experimentally fully reconstructable clean final states. We find that the "golden signals" for LQ discovery are the QCD pair production of the charge $+2 / 3$ LQ (denoted $\Psi_{1}$ ), with its decays to a charged lepton plus a down-type quark $\left(d_{i}, i=1-3\right)$. After applying a series of judicious cuts to suppress SM backgrounds that would produce the same final state, we find that one could expect on the order of 1000 events with $100 \mathrm{fb}^{-1}$ integrated luminosity for a 400-600 GeV LQ. By examining the lepton flavor structure of the observed events, one could further test the model prediction for the neutrino mass spectrum. In particular, $b$-flavor tagging will be useful in helping to distinguish the neutrino mass pattern and to probe an unknown Majorana phase in the "Inverted Hierarchy" or the "Quasi-Degenerate" scenario. Assuming that one will have knowledge of the neutrino mass spectrum in the near future, obtained from either searches for neutrinoless 
double $\beta$-decay or future long baseline neutrino oscillation studies, the possible collider discovery analyzed here would not only provide a crucial consistency check for the neutrino mass pattern, but also indicate a neutrino mass generation mechanism at a fundamental level.

Our study is organized in the remainder of the paper as follows: In Section II we present the properties of the LQs in the model. In Section III the constraints on the physical couplings and parameters coming from neutrino oscillation experiments, rare processes and EWPO are investigated. The predictions for leptoquarks decays are discussed in Section IV. Taking into account the effects of neutrino mass and mixing we show the unique predictions for the branching fractions of all leptonic decays $\Psi_{1} \rightarrow d_{i} e_{j}^{+}$and $\Psi_{2} \rightarrow d_{i} \bar{\nu}$. The impact of the Majorana phases is also studied. The pair and associated production mechanisms, cuts necessary for SM background suppression, and the signal observability at the LHC are investigated in Section V. We summarize our results in Section VI.

\section{LIGHT LEPTOQUARKS AND GRAND UNIFICATION}

A general classification of the properties of leptoquarks can be found in Ref. [4]. Here, we concentrate on the specific model realization of Ref. [6] that consists of a simple realistic extension of the Georgi-Glashow model [10] in which the light neutrino masses are generated through the Type II seesaw mechanism. The Higgs sector of this model is composed of $\mathbf{5}_{\mathbf{H}}, \mathbf{2} \mathbf{4}_{\mathbf{H}}$ and $\mathbf{1} \mathbf{5}_{\mathbf{H}}$, and the matter fields live in the $\overline{\mathbf{5}}=\left(d^{C}, l\right)_{L}$ and $\mathbf{1 0}=\left(u^{C}, Q, e^{C}\right)_{L}$ representations, where $l^{T}=(\nu, e)$ and $Q^{T}=(u, d)$. As emphasized in Section [1. one of the main features of this GUT is the possibility of having light leptoquarks consistent with all constraints coming from the proton lifetime and the unification of gauge couplings $[6,6$, 9]. Here, we focus on the properties of these light scalar leptoquarks

$$
\Phi_{b}^{T}=\left(\Psi_{1}, \Psi_{2}\right) \sim(\mathbf{3}, \mathbf{2}, 1 / 6)
$$

The electric charges of the leptoquarks are $Q\left(\Psi_{1}\right)=I_{3}+Y=2 / 3$ and $Q\left(\Psi_{2}\right)=-1 / 3$, respectively. The LQ $\Phi_{b}$ lives in the $\mathbf{1 5}_{\mathbf{H}}$ representation together with $\Delta \sim(1,3,1)$, the scalar triplet field responsible for implementing the Type II seesaw mechanism. Using the interactions in the model [6] one finds

$$
Y_{\nu} \overline{\mathbf{5}} \overline{\mathbf{5}} \mathbf{1 5} \mathbf{H}_{\mathbf{H}} \supset Y_{\nu}\left[l_{L}^{T} C i \sigma_{2} \Delta l_{L}+\sqrt{2} \bar{d}_{R} l_{L}^{\alpha} \Phi_{b}^{\beta} \epsilon_{\alpha \beta}\right],
$$

where we have suppressed the generation indices on the lepton and quark fields and Yukawa coupling matrix $Y_{\nu}$.

It is important to notice that the $S U(5)$ symmetry of the theory implies that the coupling of the lepto- 
quark to matter is defined by the mass matrix for neutrinos

$$
Y_{\nu}=\frac{M_{\nu}}{\sqrt{2} v_{\Delta}}
$$

where $v_{\Delta} / \sqrt{2}$ is the vacuum expectation value of the neutral component of the field $\Delta$. As shown in Ref. [6] the leptoquark $\Phi_{b}$ has negative (positive) contribution to $b_{1}-b_{2}\left(b_{2}-b_{3}\right)$, where $b_{i}$ stands for the different beta function coefficients for the three SM gauge groups. Therefore, the presence of this field helps to achieve unification without supersymmetry. If the LQ mass lies in the range of $100 \mathrm{GeV}-1 \mathrm{TeV}$, one obtains gauge coupling unification in agreement with experimental observations at the low energies.

The Lagrangian relevant for our study is given by:

$$
\mathcal{L}=\left(D_{\mu} \Phi_{b}\right)^{\dagger}\left(D^{\mu} \Phi_{b}\right)+\mathcal{L}_{Y}-V
$$

where

$$
\begin{aligned}
\mathcal{L}_{Y} & =-\left(\sqrt{2} \bar{d}_{R} Y_{\nu} l_{L}^{\alpha} \Phi_{b}^{\beta} \epsilon_{\alpha \beta}+\text { h.c. }\right) \\
V & =M_{\Phi_{b}}^{2} \Phi_{b}^{\dagger} \Phi_{b}+\lambda_{1}\left(\Phi_{b}^{\dagger} \Phi_{b}\right)\left(H^{\dagger} H\right)+\lambda_{2}\left(\Phi_{b}^{\dagger} H\right)\left(H^{\dagger} \Phi_{b}\right)+\lambda_{3}\left(\Phi_{b}^{\dagger} \Phi_{b}\right)^{2}
\end{aligned}
$$

where $H^{T}=\left(H^{+}, H^{0}\right)$ is the SM Higgs doublet. The tree-level masses of the leptoquarks, after the electroweak symmetry breaking $\left\langle H^{0}\right\rangle=v_{0} / \sqrt{2}$, are given by:

$$
M_{\Psi_{1}}^{2}=M_{\Phi_{b}}^{2}+\lambda_{1} v_{0}^{2} / 2 \quad \text { and } \quad M_{\Psi_{2}}^{2}=M_{\Psi_{1}}^{2}+\lambda_{2} v_{0}^{2} / 2
$$

In principle, the dimensionless couplings $\lambda_{1}, \lambda_{2}$ can be either positive, negative, or zero ${ }^{1}$. Thus, we have no a priori prediction for which of the two LQs is the lightest ${ }^{2}$. In our study of the collider phenomenology, we will focus on the case in which $M_{\Psi_{2}}-M_{\Psi_{1}} \geq 0$, corresponding to $\lambda_{2} \geq 0$.

Working in the basis of physical fermions, the new LQ-matter Yukawa interactions read:

$$
\bar{d}_{R} \Gamma_{1} e_{L} \Psi_{1} \quad \text { and } \quad \bar{d}_{R} \Gamma_{2} \nu_{L} \Psi_{2},
$$

where

$$
\Gamma_{1}=\sqrt{2} D_{R}^{\dagger} Y_{\nu} E_{L}=\Gamma_{2} V_{P M N S}^{\dagger} K_{3}^{*} \text { and } \Gamma_{2}=\sqrt{2} D_{R}^{\dagger} Y_{\nu} N_{L}=\sqrt{2} B K_{3}^{*} V_{P M N S}^{*} Y_{\nu}^{\text {diag }} .
$$

Here, $V_{P M N S}$ is the leptonic (neutrino) mixing matrix; $D_{R}, E_{L}$, and $N_{L}$ transform the right-handed down quark, left-handed electron, and left-handed neutrino mass eigenstates into the corresponding flavor states;

\footnotetext{
${ }^{1}$ For negative $\lambda_{1}$ and/or $\lambda_{2}$, boundedness of the potential restricts the magnitude of coupling relative to that of $\lambda_{3}$ and the SM Higgs quartic coupling.

${ }^{2}$ We note that SM radiative corrections will generate a mass splitting[11] $M_{\Psi_{1}}-M_{\Psi_{2}} \approx 106 \mathrm{MeV}$ in the $\overline{\mathrm{MS}}$ scheme.
} 
and $K_{3}$ is a diagonal matrix containing the unknown phases

$$
K_{3}=\left(\begin{array}{lll}
e^{i \alpha_{1}} & 0 & 0 \\
0 & e^{i \alpha_{2}} & 0 \\
0 & 0 & e^{i \alpha_{3}}
\end{array}\right)
$$

The other mixing matrix, $B$, appearing in Eq. (9) involves the product of RH quark and LH charged lepton rotation matrices: is $B=D_{R}^{\dagger} E_{L}^{*}$. It has been pointed out [9, 12, 13] that in order to satisfy the constraints from proton decay, the unitary matrix $B$ must be of the form

$$
B=\left(\begin{array}{lll}
0 & 0 & e^{i \beta_{1}} \\
0 & e^{i \beta_{2}} & 0 \\
e^{i \beta_{3}} & 0 & 0
\end{array}\right)
$$

Once we impose the constraints from the lower limit on the proton lifetime, the LQ-matter couplings are dictated by the neutrino masses and mixing, as presented in detail in Appendix A. It is useful to notice that the Yukawa couplings scale parametrically as $\Gamma_{1,2} \propto m_{\nu} / v_{\Delta}$. We summarize the interactions for the leptoquarks in Table. [.

\section{CONSTRAINTS ON THE PHYSICAL PARAMETERS}

In this section we discuss the constraints from neutrino oscillation experiments, rare decays and collider experiments on the Yukawa couplings and the physical parameters $M_{\Psi_{1}}, M_{\Psi_{2}}$ and $v_{\Delta}$ in this theory.

\section{A. Constraints From Neutrino Oscillation Experiments}

The physical Yukawa couplings of leptoquarks for the leptonic decays are given by Eq. (9). In order to understand the constraints from neutrino physics we start from the correlation between the neutrino masses and mixing angles. The leptonic mixing matrix is given by

$$
V_{P M N S}=\left(\begin{array}{lll}
c_{12} c_{13} & c_{13} s_{12} & e^{-\mathrm{i} \delta} s_{13} \\
-c_{12} s_{13} s_{23} e^{\mathrm{i} \delta}-c_{23} s_{12} & c_{12} c_{23}-e^{\mathrm{i} \delta} s_{12} s_{13} s_{23} & c_{13} s_{23} \\
s_{12} s_{23}-e^{\mathrm{i} \delta} c_{12} c_{23} s_{13} & -c_{23} s_{12} s_{13} e^{\mathrm{i} \delta}-c_{12} s_{23} & c_{13} c_{23}
\end{array}\right) \times \operatorname{diag}\left(e^{i \Phi_{1} / 2}, 1, e^{i \Phi_{2} / 2}\right),(1
$$

where $s_{i j}=\sin \theta_{i j}, c_{i j}=\cos \theta_{i j}, 0 \leq \theta_{i j} \leq \pi / 2$ and $0 \leq \delta \leq 2 \pi$. The phase $\delta$ is the Dirac CP phase, and $\Phi_{i}$ are the Majorana phases. The experimental constraints on the neutrino masses and mixing parameters, 


\begin{tabular}{|c|c|c|}
\hline Fields & Vertices & Couplings \\
\hline$\Psi_{i}(i=1,2)$ & $\bar{d} \Psi_{i} l_{i}$ & $i \Gamma_{i} P_{L}$ \\
\hline Yukawa & $l_{1}=e, l_{2}=\nu$ & $P_{L}=\left(1-\gamma_{5}\right) / 2$ \\
\hline \multirow[t]{2}{*}{ QCD } & $\Psi_{i}\left(p_{1}\right) \Psi_{i}^{*}\left(p_{2}\right) G_{\mu}^{a}$ & $i g_{s} \frac{\lambda_{a}}{2}\left(p_{1}-p_{2}\right)_{\mu}$ \\
\hline & $\Psi_{i} \Psi_{i}^{*} G_{\mu}^{a} G^{b \mu}$ & $i g_{s}^{2} \frac{\lambda_{a}}{2} \frac{\lambda_{b}}{2}$ \\
\hline \multirow[t]{5}{*}{ 3-point EW } & $\Psi_{i}\left(p_{1}\right) \Psi_{i}^{*}\left(p_{2}\right) A_{\mu}$ & $i Q_{i} e\left(p_{1}-p_{2}\right)_{\mu}$ \\
\hline & & $Q_{1}=2 / 3, Q_{2}=-1 / 3$ \\
\hline & $\Psi_{i}\left(p_{1}\right) \Psi_{i}^{*}\left(p_{2}\right) Z_{\mu}$ & $i \frac{g_{2}}{6} \cos \theta_{W} K_{i}\left(p_{1}-p_{2}\right)_{\mu}$ \\
\hline & & $K_{1}=4-\frac{1}{\cos ^{2} \theta_{W}}, K_{2}=-2-\frac{1}{\cos ^{2} \theta_{W}}$ \\
\hline & $\Psi_{1}\left(p_{1}\right) \Psi_{2}^{*}\left(p_{2}\right) W_{\mu}^{-}$ & $-i \frac{g_{2}}{\sqrt{2}}\left(p_{1}-p_{2}\right)_{\mu}$ \\
\hline \multirow[t]{8}{*}{ 4-point EW } & $Z_{\mu} Z_{\nu} \Psi_{i} \Psi_{i}^{*}$ & $i G_{Z Z i i} g_{\mu \nu}$ \\
\hline & & $G_{Z Z 11}=\frac{\left(g_{1}^{2}-3 g_{2}^{2}\right)^{2}}{18\left(g_{1}^{2}+g_{2}^{2}\right)}, G_{Z Z 22}=\frac{\left(g_{1}^{2}+3 g_{2}^{2}\right)^{2}}{18\left(g_{1}^{2}+g_{2}^{2}\right)}$ \\
\hline & $A_{\mu} A_{\nu} \Psi_{i} \Psi_{i}^{*}$ & $i G_{A A i i} g_{\mu \nu}$ \\
\hline & & $G_{A A 11}=\frac{8 g_{1}^{2} g_{2}^{2}}{9\left(g_{1}^{2}+g_{2}^{2}\right)}, G_{A A 22}=\frac{2 g_{1}^{2} g_{2}^{2}}{9\left(g_{1}^{2}+g_{2}^{2}\right)}$ \\
\hline & $Z_{\mu} A_{\nu} \Psi_{i} \Psi_{i}^{*}$ & $i G_{Z A i i} g_{\mu \nu}$ \\
\hline & & $G_{Z A 11}=\frac{-2 g_{1} g_{2}\left(g_{1}^{2}-3 g_{2}^{2}\right)}{9\left(g_{1}^{2}+g_{2}^{2}\right)}, G_{Z A 22}=\frac{g_{1} g_{2}\left(g_{1}^{2}+3 g_{2}^{2}\right)}{9\left(g_{1}^{2}+g_{2}^{2}\right)}$ \\
\hline & $W_{\mu}^{+} W_{\nu}^{-} \Psi_{i} \Psi_{i}^{*}$ & $i G_{W W i i} g_{\mu \nu}$ \\
\hline & & $G_{W W 11}=G_{W W 22}=g_{2}^{2} / 2$ \\
\hline
\end{tabular}

TABLE I: Feynman rules for the leptoquarks Yukawa and gauge interactions. The momenta are all assumed to be incoming.

at $2 \sigma$ level are [14]

$$
\begin{aligned}
7.3 \times 10^{-5} \mathrm{eV}^{2} & <\Delta m_{21}^{2}<8.1 \times 10^{-5} \mathrm{eV}^{2}, \\
2.1 \times 10^{-3} \mathrm{eV}^{2}<\left|\Delta m_{31}^{2}\right| & <2.7 \times 10^{-3} \mathrm{eV}^{2}, \\
0.28 & <\sin ^{2} \theta_{12}<0.37 \\
0.38< & <\sin ^{2} \theta_{23}<0.63 \\
\sin ^{2} \theta_{13} & <0.033
\end{aligned}
$$

and $\sum_{i} m_{i}<1.2 \mathrm{eV}$. Following the convention, we denote the case $\Delta m_{31}^{2} \equiv m_{3}^{2}-m_{1}^{2}>0$ as the normal hierarchy $(\mathrm{NH})$ and otherwise the inverted hierarchy $(\mathrm{IH})$.

In this section we neglect all the phases. The observed neutrino mass spectra indicate that the neutrino mass matrix

$$
M_{\nu}=V_{P M N S}^{*} m_{\nu}^{\operatorname{diag}} V_{P M N S}^{\dagger}
$$



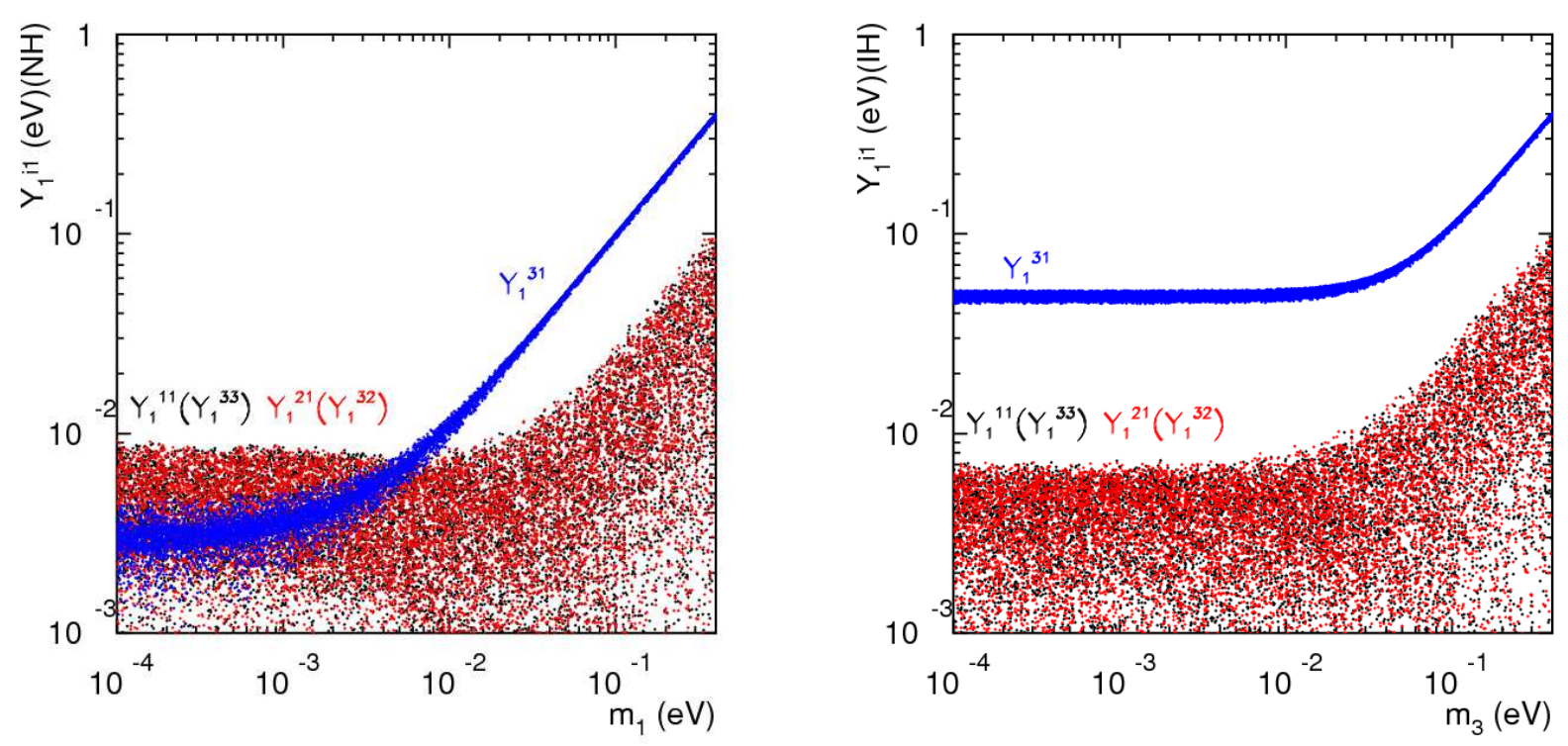

FIG. 1: Constraints on the leptoquark couplings $Y_{1}^{i 1}=\Gamma_{1}^{i 1} \times v_{\Delta}$ versus the lowest neutrino mass for NH (left) and IH (right) when all the phases vanish. Due to the symmetry as in Eq. 20), the equal couplings are also indicated in the parenthesis.

presents the following patterns

$$
\begin{array}{lll} 
& M_{\nu}^{11} \ll M_{\nu}^{22}, M_{\nu}^{33} \quad \text { for } \mathrm{NH}, \\
& M_{\nu}^{11}>M_{\nu}^{22}, M_{\nu}^{33} \quad \text { for } \mathrm{IH}, \\
\text { and } & M_{\nu}^{23}>M_{\nu}^{12}, M_{\nu}^{13} \quad \text { for both NH, IH. }
\end{array}
$$

where the last relation for the off-diagonal elements is due to the large atmospheric mixing angle $\theta_{23}$. See for example Ref. [15] for more detailed discussions.

In order to see the relationship between neutrino mass pattern and the Yukawa couplings of the leptoquarks, we first introduce a dimensionful coupling matrix relevant to $\Psi_{1}$ Yukawa coupling

$$
Y_{1}=\Gamma_{1} \times v_{\Delta}=\left(\begin{array}{ccc}
0 & 0 & 1 \\
0 & 1 & 0 \\
1 & 0 & 0
\end{array}\right) M_{\nu}, \quad \text { or } \quad Y_{1}^{i j}=M_{\nu}^{(4-i), j}
$$

Here we have neglected the phases in the B matrix. Note that the neutrino mass matrix $M_{\nu}$ is symmetric but $Y_{1}$ is not, with the first index $i$ for the down-type quarks and the second index $j$ for the charged leptons.

We are thus able to determine all the Yukawa couplings that are consistent with a given set of neutrino masses and oscillation parameters. To illustrate this relationship, we perform a numerical scan over the 

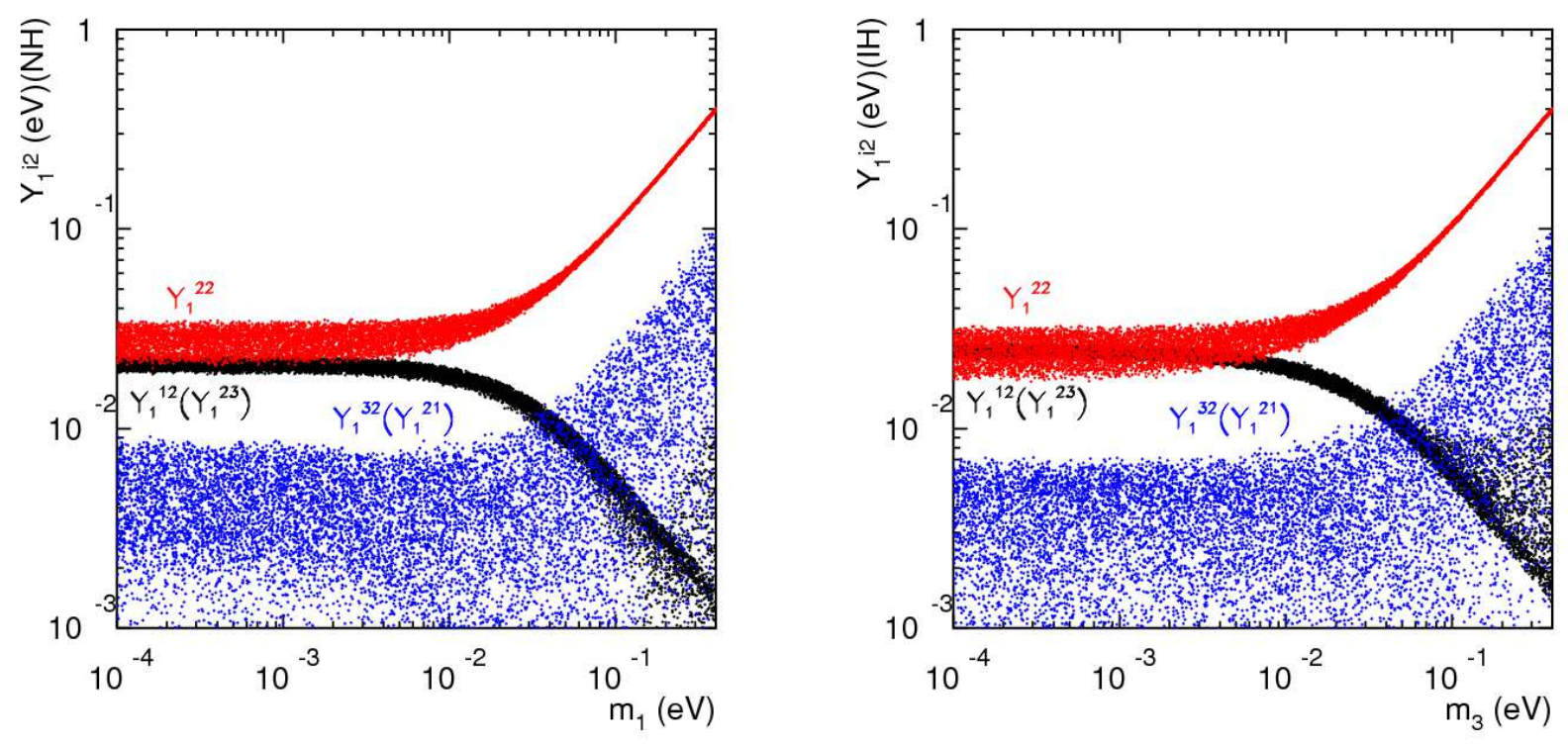

FIG. 2: Constraints on the leptoquark couplings $Y_{1}^{i 2}=\Gamma_{1}^{i 2} \times v_{\Delta}$ versus the lowest neutrino mass for NH (left) and IH (right) when all the phases vanish. Due to the symmetry as in Eq. 20], the equal couplings are also indicated in the parenthesis.

neutrino masses and mixing angles, using a uniform, random distribution while taking into account the above experimental constraints and neglecting all phases. We show the scatter plots for the allowed values for the couplings of each lepton flavor in Figs. 1, 2] and 3 versus the lightest neutrino mass in each spectrum, the normal hierarchy (left panels) and the inverted hierarchy (right panels). We see two distinctive regions in terms of the lightest neutrino mass. In the case $m_{1(3)}<10^{-1} \mathrm{eV}$,

$$
\begin{array}{ll}
\text { Fig. 1 for } e: & Y_{1}^{31} \simeq Y_{1}^{11}, Y_{1}^{21} \text { for } \mathrm{NH}, \\
& Y_{1}^{31} \gg Y_{1}^{11}, Y_{1}^{21} \text { for } \mathrm{IH},
\end{array}
$$

Fig. 2for $\mu: \quad Y_{1}^{22} \gtrsim Y_{1}^{12}>Y_{1}^{32}$ for both $\mathrm{NH}$ and $\mathrm{IH}$,

Fig. [3 for $\tau: \quad Y_{1}^{13} \gtrsim Y_{1}^{23}>Y_{1}^{33}$ for both $\mathrm{NH}$ and $\mathrm{IH}$.

On the other hand, for $m_{1(3)}>10^{-1} \mathrm{eV}$, we have the quasi-degenerate spectrum $M_{\nu}^{11} \approx M_{\nu}^{22} \approx M_{\nu}^{33}$, and the leading couplings in each lepton flavor are

$$
Y_{1}^{31} \approx Y_{1}^{22} \approx Y_{1}^{13}
$$

As for the $\Psi_{2}$ decays, we sum over the final state neutrinos since they are experimentally unobservable. 

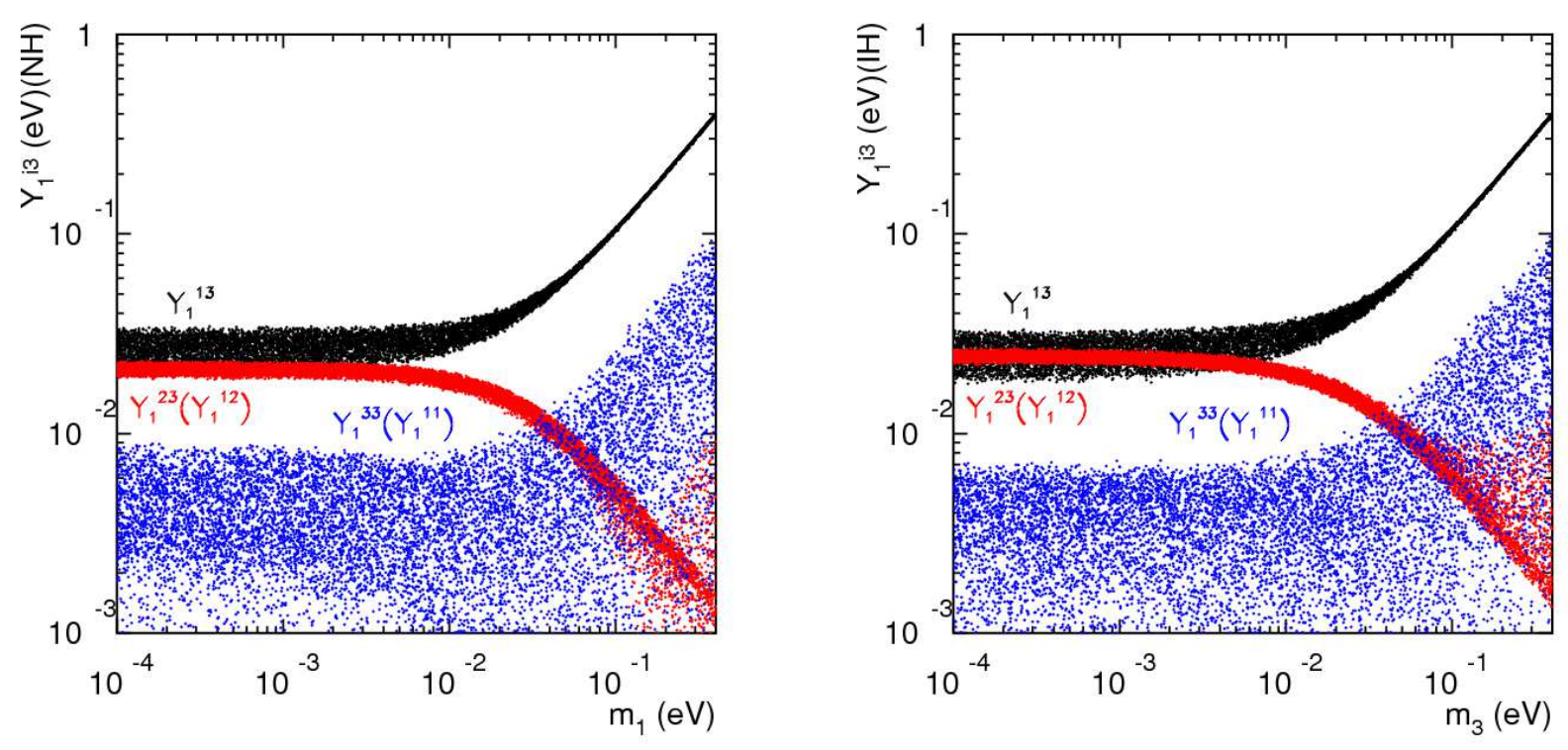

FIG. 3: Constraints on the leptoquark couplings $Y_{1}^{i 3}=\Gamma_{1}^{i 3} \times v_{\Delta}$ versus the lowest neutrino mass for NH (left) and $\mathrm{IH}$ (right) when all the phases vanish. Due to the symmetry as in Eq. 201, the equal couplings are also indicated in the parentheses.

Thus the relevant couplings are written as

$$
Y_{2}^{i}=\sum_{j=1}^{3}\left|\Gamma_{2}^{i j}\right|^{2} \times v_{\Delta}^{2} \quad(i=1,2,3 \text { for down quarks } d, s, b) .
$$

The allowed values are shown in Fig. 4. In this case, $Y_{2}^{3} \ll Y_{2}^{1}, Y_{2}^{2}$ in the $\mathrm{NH}$ and $Y_{2}^{3}>Y_{2}^{1}, Y_{2}^{2}$ in the IH when the lightest neutrino mass is smaller than $10^{-2} \mathrm{eV}$. The explicit expressions of $Y_{1}$ and $Y_{2}$ obtained via $\Gamma_{1}$ and $\Gamma_{2}$ are collected in Appendix A.

\section{B. Constraints from Rare Decays}

Here we discuss the constraints coming from meson decays, meson-antimeson mixing and lepton flavor violating processes. The most important constraints for the leptoquark parameters are from the $K_{L}^{0}$ decays to dileptons [16, 17, 18] and searches for $\mu-e$ conversion in nuclei [16]. 

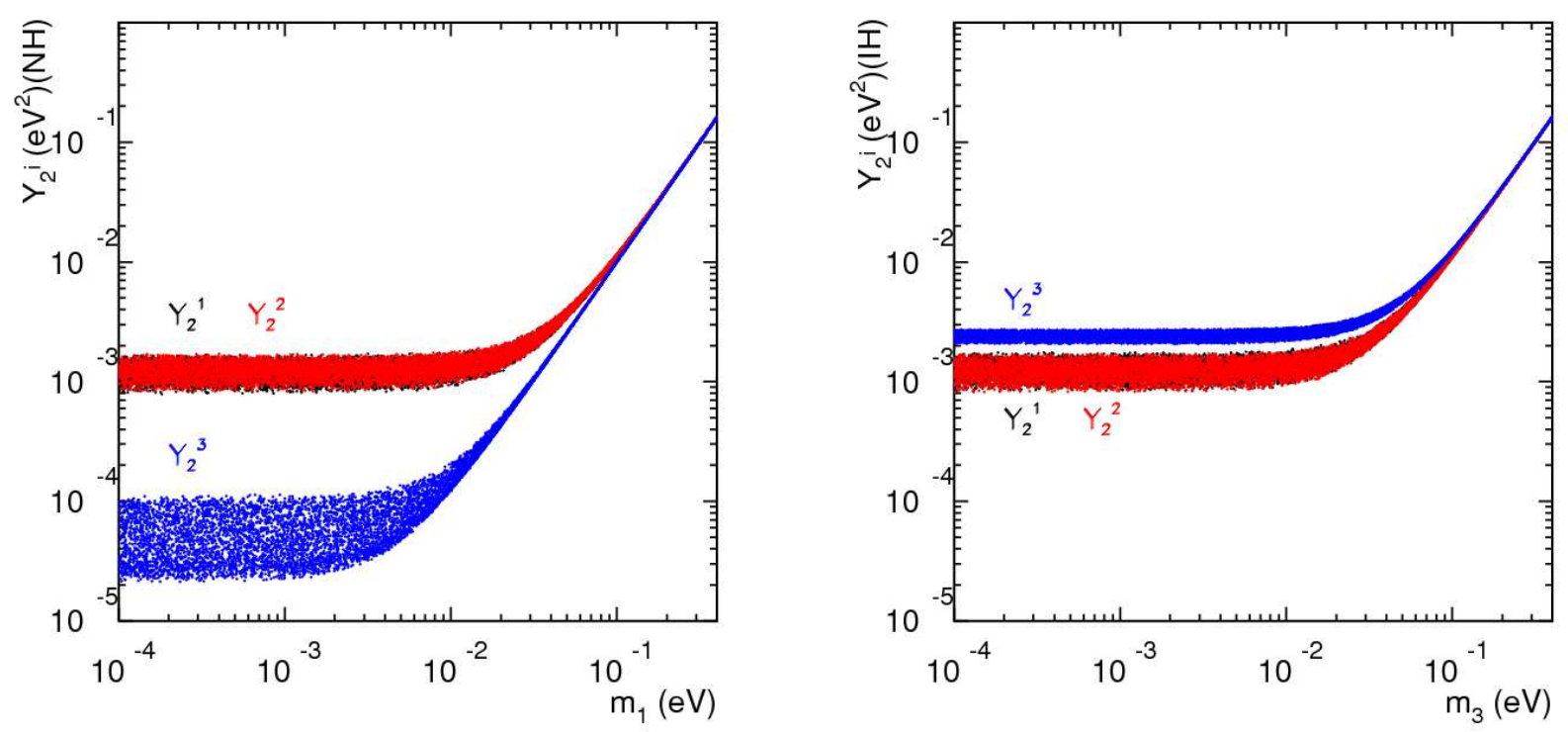

FIG. 4: Constraints on $Y_{2}^{i}=\sum_{j=1}^{3}\left|\Gamma_{2}^{i j}\right|^{2} \times v_{\Delta}^{2}$ versus the lowest neutrino mass for NH (left) and IH (right) when all the phases vanish.

\begin{tabular}{|c|c|c|}
\hline Mesons & Final states & Branching fraction \\
\hline$K_{L}^{0}$ & $\mu^{+} \mu^{-}$ & $(6.84 \pm 0.11) \times 10^{-9}$ \\
& $e^{+} e^{-}$ & $\left(0.087_{-0.041}^{+0.057}\right) \times 10^{-10}$ \\
& $e^{-} \mu^{+}$ & $<4.7 \times 10^{-12}$ \\
\hline
\end{tabular}

TABLE II: Experimental Constraints on $K_{L}^{0}$ pure leptonic decays [16].

\section{1. $K_{L}$ decays}

The meson leptonic decay rate is given by

$$
\Gamma\left(M\left(\bar{q}^{j} q^{n}\right) \rightarrow \ell^{r} \bar{\ell}^{i}\right)=\frac{k}{4 \pi} \frac{1}{M_{\Psi_{1}}^{4}} \frac{\left|\Gamma_{1}^{n r}\right|^{2}}{2} \frac{\left|\Gamma_{1}^{j i}\right|^{2}}{2}\left(\frac{f_{M}}{2}\right)^{2}\left(E_{i} E_{r}-k^{2}\right),
$$

where

$$
E_{i, r}=\sqrt{m_{i, r}^{2}+k^{2}}, \quad k=\frac{m_{M}}{2} \lambda^{1 / 2}\left(1, \frac{m_{i}^{2}}{m_{M}^{2}}, \frac{m_{r}^{2}}{m_{M}^{2}}\right) .
$$

For $K_{L}^{0}$ decay, we take $m_{K}=497.648 \mathrm{MeV}, f_{K}=160 \mathrm{MeV}$ and $\tau_{K_{L}}=5.116 \times 10^{-8} \mathrm{~s}$ [16]. We list the most precise results for the $K_{L}^{0}$ decay branching fractions $B\left(K_{L} \rightarrow X\right)$ in Table II In the case of the $\mu^{+} \mu^{-}$and $e^{+} e^{-}$final states, SM expectations involve the sum of short-distance (SD) and long-distance 
(LD) contributions [19, 20]. The latter are dominant, as the predicted short-distance contribution gives roughly one sixth of the measured branching ratio. Using chiral perturbation theory to perform a modelindependent analysis of the long-distance contributions, the authors of Ref. [19] find that they are nearly saturated by the lowest order (1.o.) term in the chiral expansion that is fixed from the measured $K_{L} \rightarrow \gamma \gamma$ branching fraction. The sub-leading long-distance contribution depends on an a priori unknown scaledependent low-energy constant $\chi(\mu)$ whose value is obtained from analysis of $K_{L} \rightarrow \mu^{+} \mu^{-}, \eta \rightarrow \mu^{+} \mu^{-}$, and $\pi^{0} \rightarrow e^{+} e^{-}$decays $^{3}$. Of these, $B\left(K_{L} \rightarrow \mu^{+} \mu^{-}\right)$is known most precisely. The difference between its experimental value and the sum of the short-distance and leading order long-distance contributions

$$
\Delta_{K}(\mu \mu) \equiv B\left(K_{L} \rightarrow \mu^{+} \mu^{-}\right)^{\exp }-\left[B\left(K_{L} \rightarrow \mu^{+} \mu^{-}\right)^{\mathrm{SD}}+B\left(K_{L} \rightarrow \mu^{+} \mu^{-}\right)_{\text {l.o. }}^{\mathrm{LD}}\right]
$$

determines the value of $\chi$ implied by this decay.

Any non-SM contribution to $K_{L} \rightarrow \mu^{+} \mu^{-}$would enter this difference and, thus, affect the extracted value of $\chi$. So long as the non-SM contribution is smaller than the uncertainty in $\Delta_{K}(\mu \mu)$, it could be absorbed by a shift in $\chi$ within its error bars without appreciably affecting the analysis of $K_{L}, \pi^{0}$, and $\eta$ decays into $\ell^{+} \ell^{-}$. The uncertainty $\delta \Delta_{K}$ is dominated by the theoretical uncertainty in $B\left(K_{L} \rightarrow \mu^{+} \mu^{-}\right)^{\mathrm{SD}}$ :

$$
\delta \Delta_{K}(\mu \mu) \simeq( \pm 0.6) \times 10^{-9}
$$

To be conservative, we thus require that the LQ contribution to $B\left(K_{L} \rightarrow \mu^{+} \mu^{-}\right)$be smaller than the magnitude of $\delta \Delta_{K}(\mu \mu)$ given in Eq. (26).

Once the constant $\chi$ has been obtained from the other processes discussed above, it is possible to make a model-independent prediction for the $K_{L} \rightarrow e^{+} e^{-}$branching fraction. In this case, however, the comparison of the experimental result with the Standard Model prediction, $\delta \Delta_{K}(e e)$, is dominated by the experimental error in $B\left(K_{L} \rightarrow e^{+} e^{-}\right)$. Consequently, we require that the LQ contribution to this channel be less than the average of the upper and lower errors quoted in Table $\amalg$

In Figs. 5 and 6, we show the branching fractions for $K_{L}^{0} \rightarrow e^{+} e^{-}, \mu^{+} \mu^{-}$for the $\mathrm{NH}$ and $\mathrm{IH}$, respectively, taking into account the leptoquark $\Psi_{1}$ contribution versus the lightest neutrino mass for $M_{\Psi_{1}} v_{\Delta}>1200 \mathrm{GeV} \cdot \mathrm{eV}$ (left panel) and $1600 \mathrm{GeV} \cdot \mathrm{eV}$ (right panel). Note that the decay rate is proportional to $\left(M_{\Psi_{1}} v_{\Delta}\right)^{-4}$ since each $\Gamma^{k \ell} \sim m_{\nu} / v_{\Delta}$. We see that there exist parameter space points for which the leptoquark contribution is smaller than uncertainty in $\Delta_{K}(\ell \ell)$ as indicated by the horizontal lines for $M_{\Psi_{1}} v_{\Delta}>1600 \mathrm{GeV} \cdot \mathrm{eV}$.

In Fig. 7 we show the predictions for LQ contributions to the decay channel $e^{-} \mu^{+}$with $M_{\Psi_{1}} v_{\Delta}>800$ $\mathrm{GeV} \cdot \mathrm{eV}$. In this case, the SM prediction is highly suppressed since flavor violation arises solely through the

\footnotetext{
${ }^{3}$ The scale $\mu$ is typically chose to be the $\rho$-meson mass.
} 
neutrino sector, so any observable effect would have to arise from new physics. The experimental BR limits, indicated by the horizontal lines in the left and right panels of Fig. 7 thus translate into direct constraints on the LQ parameters. We find the BR results for this channel more spread out and, thus, less constraining than are those for the lepton flavor conserving decays. This observation leads to an important point: Although the experimental bounds on the $e \mu$ and $e e$ channels are stronger than that of $\mu \mu$, they actually provide less stringent constraints on the model parameters, due to the suppressed couplings governed by the neutrino oscillation data.

Additional constraints follow from other meson properties and low-energy semileptonic interactions, such as the mass difference between $K_{L}$ and $K_{S}: \Delta m_{K}=3.48 \times 10^{-15} \mathrm{GeV}$ [16]. The box diagrams for a leptoquark with couplings to leptons give a contribution to $\Delta m_{K}[17,18]$, that yields a constraint

$$
\left(\left(\Gamma_{1} \Gamma_{1}^{\dagger}\right)^{21}\right)^{2} \lesssim \frac{192 \pi^{2} M_{\Psi_{1}}^{2} \Delta m_{K}}{f_{K}^{2} m_{K}} \approx 5.2 \times 10^{-10} M_{\Psi_{1}}^{2} .
$$

As we have shown in the previous section when the lightest neutrino mass is smaller than $10^{-2} \mathrm{eV},\left(Y_{1} Y_{1}^{\dagger}\right)^{21}$ is smaller than $25(24) \times 10^{-4} \mathrm{eV}^{2}$ in the $\mathrm{NH}(\mathrm{IH})$ spectrum. Using these results one finds

$$
\frac{v_{\Delta}}{1 \mathrm{eV}} \gtrsim 1.05 \times\left(\frac{10^{2} \mathrm{GeV}}{M_{\Psi_{1}}}\right)^{1 / 2}
$$

This leads to a weaker bound than that obtained from the rare decays. The same constraints are valid for the case of $\Psi_{2}$. Notice that one can satisfy these bounds easily.

We emphasize that the most stringent constraints, which follow from $K_{L}^{0} \rightarrow \mu^{-} \mu^{+}$, imply that for $M_{\Psi_{1}} v_{\Delta}>1600 \mathrm{GeV} \cdot \mathrm{eV}$ it is possible to find values for the Yukawa couplings that are consistent with both neutrino oscillation data and the quantity $\Delta_{K}(\mu \mu)$. As we discuss below, one could expect to observe a significant number of LQ events at the LHC for LQ masses in the approximate range $400 \lesssim M_{\Psi_{1}} \lesssim 1000$ $\mathrm{GeV}$, or $v_{\Delta}$ lying between about two and four $\mathrm{eV}$. Since we have no independent handle on the value of $v_{\Delta}$ nor any reason to preclude values lying in this range, we conclude that consistency of an LHC LQ discovery and the flavor changing decay $K_{L}^{0} \rightarrow \mu^{-} \mu^{+}$would imply a relatively large triplet vev in this model.

\section{2. $\mu-e$ Conversion Constraints}

Searches for charged lepton flavor violation (CLFV) in processes that conserve quark flavor can impose severe constraints on new physics. In the present case, searches for $\mu$-e conversion in nuclei yield the most stringent constraints on the LQ couplings and masses. The tightest bound on the rate for this process has been obtained by the SINDRUM Collaboration[21] using the gold nucleus. The result for the conversion to 

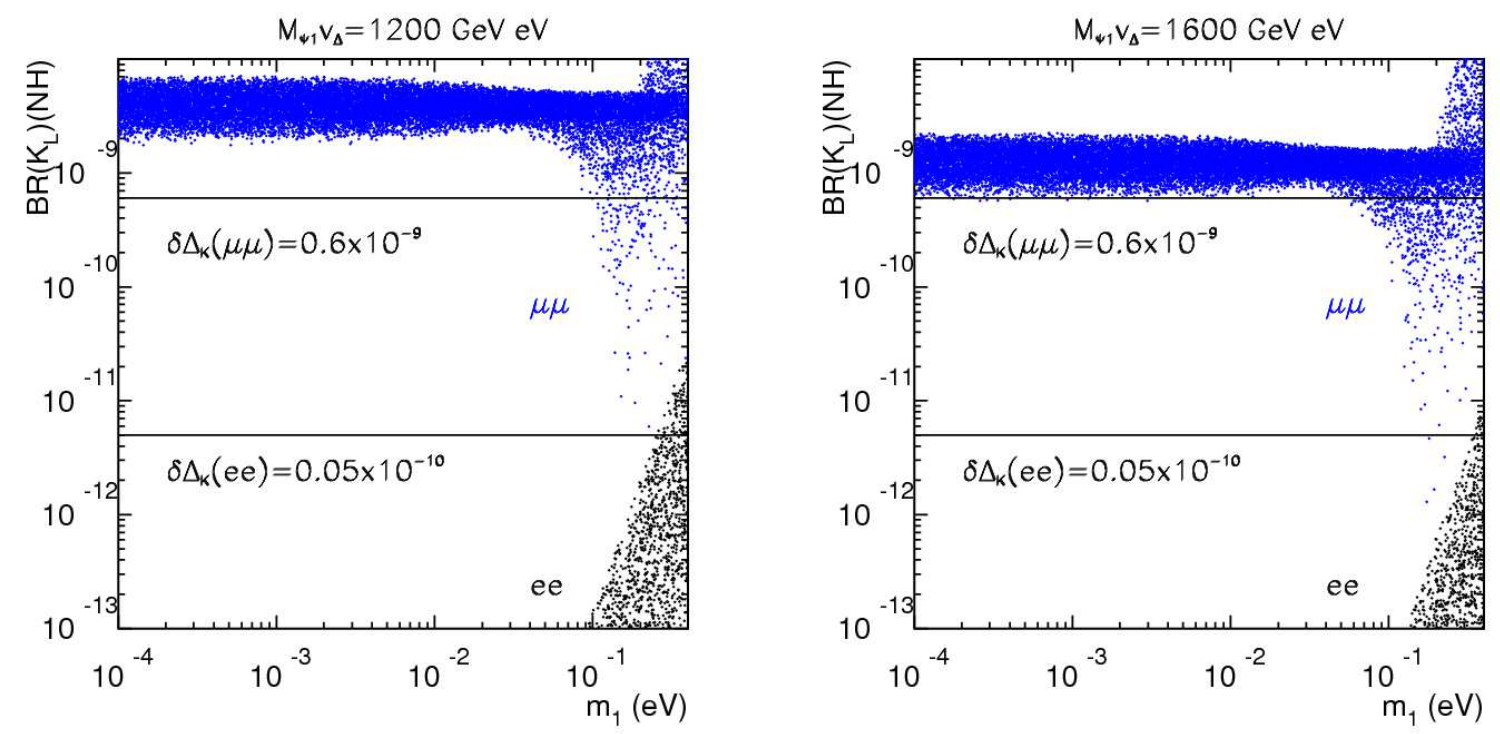

FIG. 5: Branching fractions for $K_{L}^{0} \rightarrow e^{+} e^{-}$(black) and $K_{L}^{0} \rightarrow \mu^{-} \mu^{+}$(blue) versus the lowest neutino mass for NH neglecting the phases, $M_{\Psi_{1}} v_{\Delta}=1200$ (left), 1600 (right) $\mathrm{GeV} \cdot \mathrm{eV}$. The horizontal lines represent the current $1 \sigma$ experimental bounds [16].

capture ratio $R_{\mu \rightarrow e}^{\mathrm{Au}}$ is

$$
R_{\mu \rightarrow e}^{\mathrm{Au}}=\frac{\Gamma\left[\mu^{-}+A(Z, N) \rightarrow e^{-}+A(Z, N)\right]}{\Gamma\left[\mu^{-}+A(Z, N) \rightarrow \nu+A(Z-1, N+1)\right]}<7.0 \times 10^{-13} \quad(90 \% \text { C.L. }) \quad .
$$

This ratio can be expressed in terms of the reduced conversion and capture rates $R_{\mu \rightarrow e}^{\mathrm{Au}}=\omega_{\mathrm{conv}} / \omega_{\text {capt }}$ where [22]

$$
\omega_{\mathrm{conv}}=\frac{\left|\Gamma_{1}^{11}\right|^{2}\left|\Gamma_{1}^{12}\right|^{2} m_{\mu}^{5}}{4 M_{\Psi_{1}}^{4}}\left(V^{(p)}+2 V^{(n)}\right)^{2},
$$

where $V^{(p)}$ and $V^{(n)}$ are overlap integrals involving the upper and lower components of the muon and electron wavefunctions and the proton (p) and neutron (n) number densities. Using the values for these integrals computed in Ref. [22], the experimental value for $\omega_{\text {capt }}$ and experimental limit on $R_{\mu \rightarrow e}^{\mathrm{Au}}$ shown in Table. IIII we obtain 90\% C.L. constraints on the LQ parameters as shown in Fig. 8. There we give $R_{\mu \rightarrow e}^{\mathrm{Au}}$ corresponding to lightest neutrino mass in $\mathrm{NH}$ and $\mathrm{IH}$ when $M_{\Psi_{1}} v_{\Delta}=800 \mathrm{GeV} \cdot \mathrm{eV}$. We observe that in either case, there exist values of the LQ parameters for which the predicted ratio lies below the $90 \%$ C.L. limit (indicated by the horizontal line). Although the present limits exclude large portions of parameter space, they do not preclude the model entirely. Future searches for $\mu \rightarrow e$ conversion, such as the Mu2e experiment proposed for Fermilab or the PRIME experiment at JPARC, could improve the sensitivity be several orders of magnitude. A null result from these future experiments could rule out the LQ model 

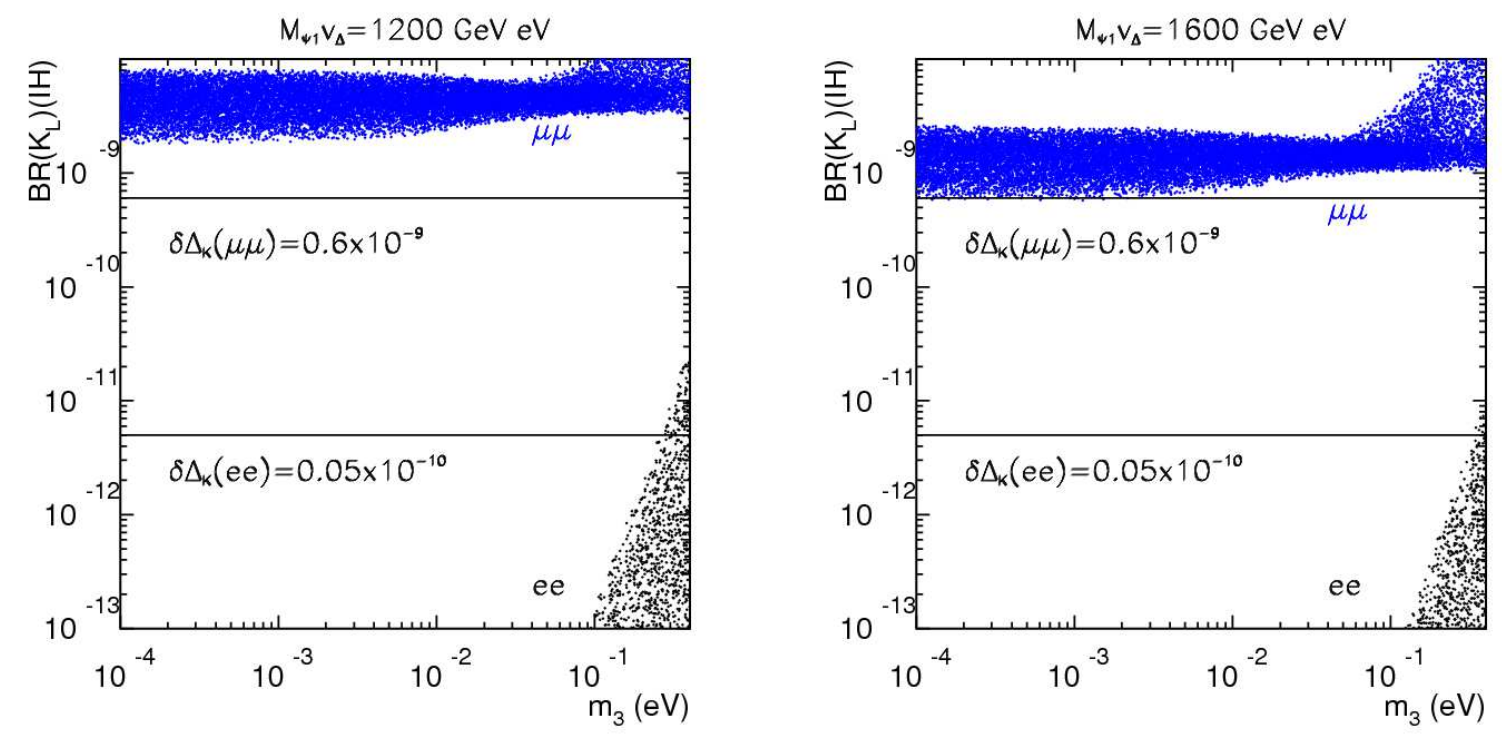

FIG. 6: Branching fractions for $K_{L}^{0} \rightarrow e^{+} e^{-}$(black) and $K_{L}^{0} \rightarrow \mu^{-} \mu^{+}$(blue) versus the lowest neutino mass for IH neglecting the phases, $M_{\Psi_{1}} v_{\Delta}=1200$ (left), 1600 (right) $\mathrm{GeV} \cdot \mathrm{eV}$. The horizontal lines represent the current $1 \sigma$ experimental bounds $\lfloor 16\rfloor$.

\begin{tabular}{|c|c|c|c|c|}
\hline Nucleus & $V^{(p)}$ & $V^{(n)}$ & $\omega_{\text {capt }}\left(10^{6} s^{-1}\right)$ & $\omega_{\text {conv }} / \omega_{\text {capt }}$ \\
\hline${ }_{79}^{197} \mathrm{Au}$ & 0.0974 & 0.146 & 13.07 & $<7 \times 10^{-13}$ \\
\hline
\end{tabular}

TABLE III: Values of the relevant parameters for $\mu-e$ conversion in Au [16, 22].

under consideration here unless the product $M_{\Psi_{1}} v_{\Delta}$ is quite heavy. We will explore this possibility in a forthcoming study.

Finally, we note that the leptoquark contribution to other lepton flavor violating processes, such as $\mu \rightarrow e \gamma$ and $\mu \rightarrow 3 e$, arise at one-loop level, and thus provide less stringent constraints.

\section{Other Constraints}

\section{Oblique Parameters}

In this section we study the constraints implied by leptoquark loop contributions to electroweak precision observables (EWPO). Since the LQ-matter field couplings are tiny due to their dependence on $Y_{\nu}$, we may safely neglect vertex, fermion propagator, and box graph loop corrections and concentrate on corrections to the gauge boson propagators. For sufficiently heavy LQs, one may characterize the leading effects of these 

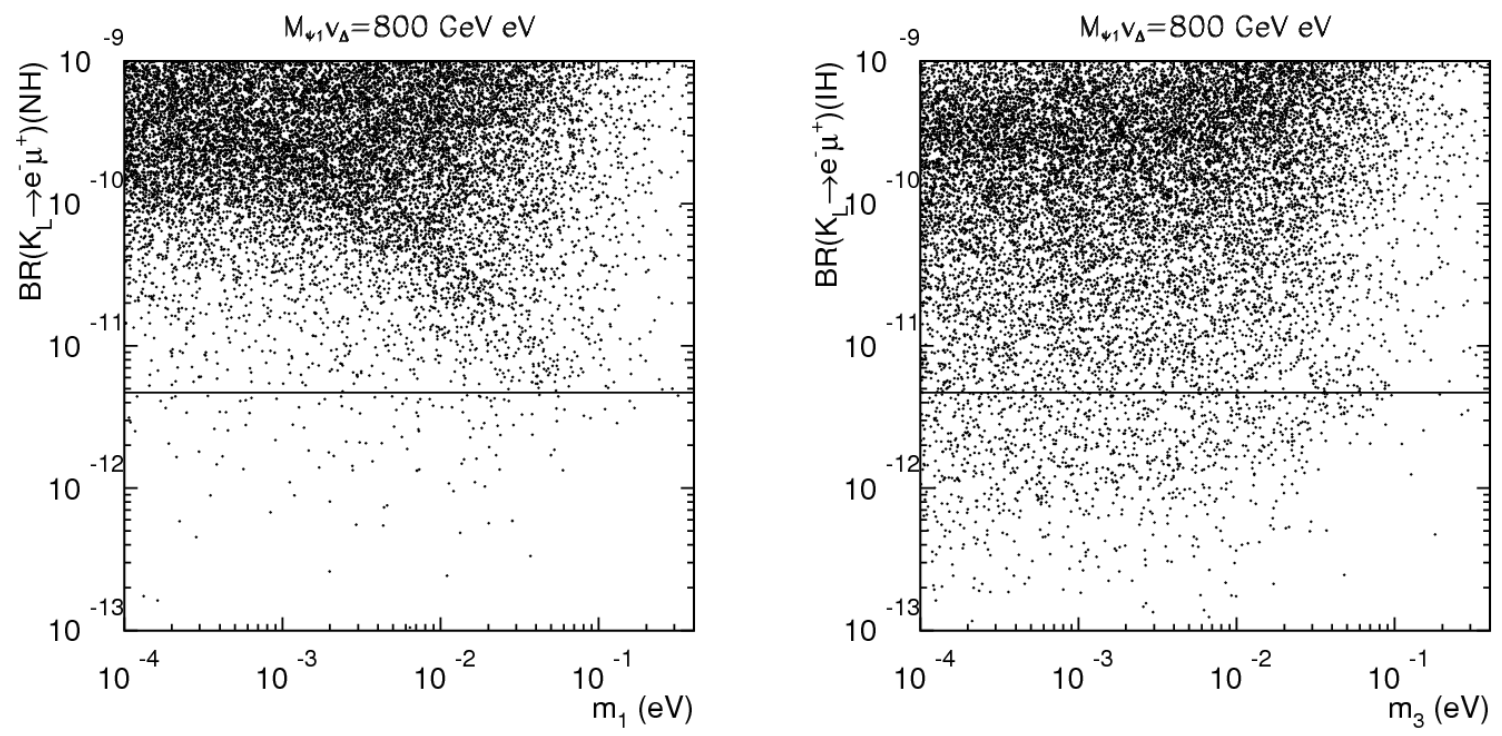

FIG. 7: Branching fractions for $K_{L}^{0} \rightarrow e^{-} \mu^{+}$versus the lowest neutrino mass for NH (left) and IH (right) neglecting the phases, $M_{\Psi_{1}} v_{\Delta}=800 \mathrm{GeV} \cdot \mathrm{eV}$. The horizontal line represents the current $1 \sigma$ experimental bound [16].

corrections in terms of the oblique parameters, $S, T$, and $U$ [23]. The $U$ parameter is typically quite small and does not add a significant constraint in this case. We have computed the full set of LQ contributions to the gauge boson self-energy functions, and the resulting contributions to $T$ and $S$ are:

$$
\begin{aligned}
\hat{\alpha}\left(M_{Z}\right) T & \equiv \frac{1}{M_{W}^{2}}\left\{\hat{\Pi}_{W W}(0)-\hat{c}^{2}\left(\hat{\Pi}_{Z Z}(0)+\frac{2 \hat{s}}{\hat{c}} \hat{\Pi}_{Z \gamma}(0)\right)\right\} \approx \frac{N_{C}}{8 \pi^{2} v^{2}}\left(-\frac{2 \Delta M^{2}}{3}\right) \\
\frac{\hat{\alpha}\left(M_{Z}\right)}{4 \hat{s}_{Z}^{2} \hat{c}_{Z}^{2}} S & \equiv \frac{1}{M_{Z}^{2}} \operatorname{Re}\left\{\hat{\Pi}_{Z Z}\left(M_{Z}^{2}\right)-\hat{\Pi}_{Z Z}(0)+\frac{\hat{c}^{2}-\hat{s}^{2}}{\hat{c} \hat{s}}\left[\hat{\Pi}_{Z \gamma}(0)-\hat{\Pi}_{Z \gamma}\left(M_{Z}^{2}\right)\right]-\hat{\Pi}_{\gamma \gamma}\left(M_{Z}^{2}\right)\right\} \\
& \approx-\frac{N_{C}}{24 \pi^{2} v^{2}}\left(\frac{M_{Z}^{2} \Delta M}{3 M_{\Psi_{1}}}\right)
\end{aligned}
$$

where $\Delta M=M_{\Psi_{2}}-M_{\Psi_{1}}, \hat{s}(\hat{c})$ is the sine (cosine) of the weak mixing angle in the $\overline{\mathrm{MS}}$ scheme, and "New" indicates the contribution from new physics $\left(\Psi_{1,2}\right)$. We have carried out renormalization at a scale $\mu=M_{Z}$. The complete expressions for LQ contributions are given in Appendix B, while in Eqs. (31,32) we give approximate expressions in the limit that the mass splitting $|\Delta M|$ and $Z$-boson mass are small compared to the LQ mass $M_{\Psi_{1}}$. The latest global fit to EWPO yields for these parameters are $S=-0.10 \pm 0.10(-0.08)$, $T=-0.08 \pm 0.11(+0.09)$, and $U=0.15 \pm 0.11(+0.01)$, assuming a value for the SM Higgs boson mass $M_{H}=117 \mathrm{GeV}(300 \mathrm{GeV})[16]$. The most important constraint is from the $T$ parameter which gives us a $1 \sigma$ bound of leptoquark mass splitting $|\Delta M| \lesssim 60 \mathrm{GeV}$. 

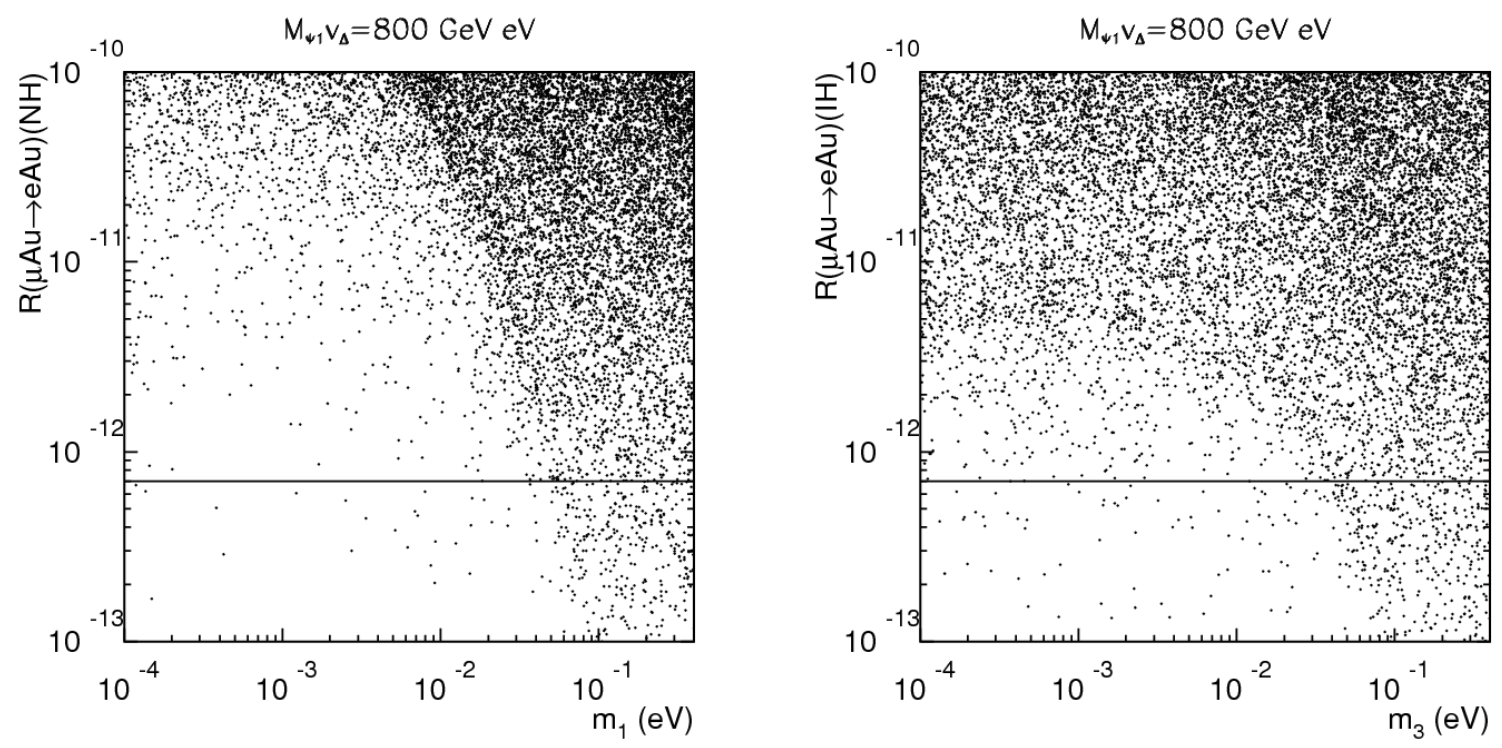

FIG. 8: The rate of $\mu-e$ conversion in Au nuclei versus the lowest neutrino mass for $\mathrm{NH}($ left) and $\mathrm{IH}$ (right) without any phases $M_{\Psi_{1}} v_{\Delta}=800 \mathrm{GeV} \cdot \mathrm{eV}$. The horizontal line represents the current $90 \%$ C.L. experimental bound [16].

\section{Collider Constraints and $\rho$-parameter}

The current constraint on the LQ mass, $M_{\Psi_{1}}$ and $M_{\Psi_{2}}$, comes from the direct searches at the Tevatron [16]

$$
M_{\Psi_{1,2}} \gtrsim 250 \mathrm{GeV} \text {. }
$$

while for the values of the triplet vev one has,

$$
1 \mathrm{eV} \lesssim v_{\Delta} \lesssim 1 \mathrm{GeV}
$$

where the lower bound follows from the assumption that $m_{\nu} \sim 1 \mathrm{eV}$ and that the Yukawa interactions are perturbative, while the upper bound is from the constraint on the electroweak $\rho$-parameter [24].

\section{LEPTOQUARK DECAYS AND NEUTRINO MASSES}

In this section we study the main features of the LQ decays taking into account the constraints on the neutrino masses and mixing. From Eq. (9) one can conclude that the leptoquark (LQ) decays could be different in each spectrum for neutrinos and we will explore this interesting connection. 


\section{A. Main Features of the Leptoquarks Decays}

The decays of the leptoquarks into fermions $\Psi_{1} \rightarrow d_{i} e_{j}^{+}$and $\Psi_{2} \rightarrow d_{i} \bar{\nu}$, where $d_{i}=d, s, b$ and $e_{j}=e, \mu, \tau$, are of most interest. The partial widths are given by

$$
\Gamma\left(\Psi_{1} \rightarrow d_{i} e_{j}^{+}\right)=\frac{\left|\Gamma_{1}^{i j}\right|^{2}}{16 \pi} M_{\Psi_{1}}, \quad \Gamma\left(\Psi_{2} \rightarrow d_{i} \bar{\nu}_{j}\right)=\frac{\left|\Gamma_{2}^{i j}\right|^{2}}{16 \pi} M_{\Psi_{2}} .
$$

The widths are proportional to the Yukawa coupling squared, governed by $m_{\nu}^{2} / v_{\Delta}^{2}$.

The other competing decay modes come from the charged current gauge interaction. Depending on the mass splitting between the leptoquarks, one has the decays of the heavy leptoquark into the lighter one plus a real $(W)$ or virtual $\left(W^{*}\right)$ gauge boson. For example, if $\Delta M=M_{\Psi_{2}}-M_{\Psi_{1}}>0$, the $\Psi_{2}$ decay rates for these processes are given by

$$
\begin{aligned}
\Gamma\left(\Psi_{2} \rightarrow \Psi_{1} W^{-}\right) & =\frac{M_{\Psi_{2}} g_{2}^{2}}{32 \pi r_{W}^{2}} \lambda^{\frac{3}{2}}\left(1, r_{W}^{2}, r_{\Psi_{1}}^{2}\right), \\
\Gamma\left(\Psi_{2} \rightarrow \Psi_{1} W^{-*} \rightarrow \Psi_{1} \pi^{-}\right) & =\frac{g_{2}^{4} V_{u d}^{2} \Delta M^{3} f_{\pi}^{2}}{32 \pi M_{W}^{4}} \sqrt{1-\frac{m_{\pi}^{2}}{\Delta M^{2}}}, \\
\Gamma\left(\Psi_{2} \rightarrow \Psi_{1} W^{-*} \rightarrow \Psi_{1} e^{-}\left(\mu^{-}\right) \bar{\nu}_{e}\left(\bar{\nu}_{\mu}\right)\right) & =\frac{g_{2}^{4} \Delta M^{5}}{480 \pi^{3} M_{W}^{4}}, \\
\Gamma\left(\Psi_{2} \rightarrow \Psi_{1} W^{-*} \rightarrow \Psi_{1} q \bar{q}^{\prime}\right) & =3 \Gamma\left(\Psi_{2} \rightarrow \Psi_{1} e^{-}\left(\mu^{-}\right) \bar{\nu}_{e}\left(\bar{\nu}_{\mu}\right)\right) .
\end{aligned}
$$

where we have omitted the CKM factor associated with the $W q \bar{q}^{\prime}$ coupling in Eq. (39) since we are considering the inclusive channels of $q \bar{q}^{\prime}$, and where $r_{i}=M_{i} / M_{\Psi_{2}}$. In Fig. 9 we show the branching fractions for the decays of $\Psi_{2}$ versus its mass with $M_{\Psi_{1}}=250 \mathrm{GeV}$ and $v_{\Delta}=5 \mathrm{eV}$ and $10 \mathrm{eV}$ in (a) and (b), respectively, assuming that the Yukawa coupling is diagonal for simplicity. We see that once the mass difference is large and a real gauge boson channel is open, then it takes over the fermionic channels. In Fig. 10 we plot the branching fractions versus $v_{\Delta}$ with $M_{\Psi_{1}}=250 \mathrm{GeV}$ and $M_{\Psi_{2}}=300 \mathrm{GeV}$. Also in the second case: $M_{\Psi_{1}}=400 \mathrm{GeV}$, and $M_{\Psi_{2}}=450 \mathrm{GeV}$. We see that for $v_{\Delta}<10 \mathrm{eV}$, the Yukawa coupling is sufficiently large so that the fermionic channels become dominant. The features are the same for $\Psi_{1}$ if it is heavier than $\Psi_{2}$. The presence of charged leptons in the final state (rather than missing energy associated with neutrinos) facilitates the use of collider observables to identify the connection between the leptoquark properties and the neutrino mass spectrum. Consequently, we will focus on the parameter region in which $M_{\Psi_{2}}>M_{\Psi_{1}}$. To further quantify this situation, we present the scatter plots in Fig. 11 in a plane of $\Delta M-v_{\Delta}$ under the condition $\Gamma\left(\Psi_{2} \rightarrow d_{i} \bar{\nu}_{j}\right)>\Gamma\left(\Psi_{2} \rightarrow \Psi_{1} W^{*}\right)$. We see that if the mass difference is small enough, there is a broad range for the vev $v_{\Delta} \sim 1-10^{6} \mathrm{eV}$, although an $\mathrm{eV}$ value may be more natural for the light neutrino mass generation. 

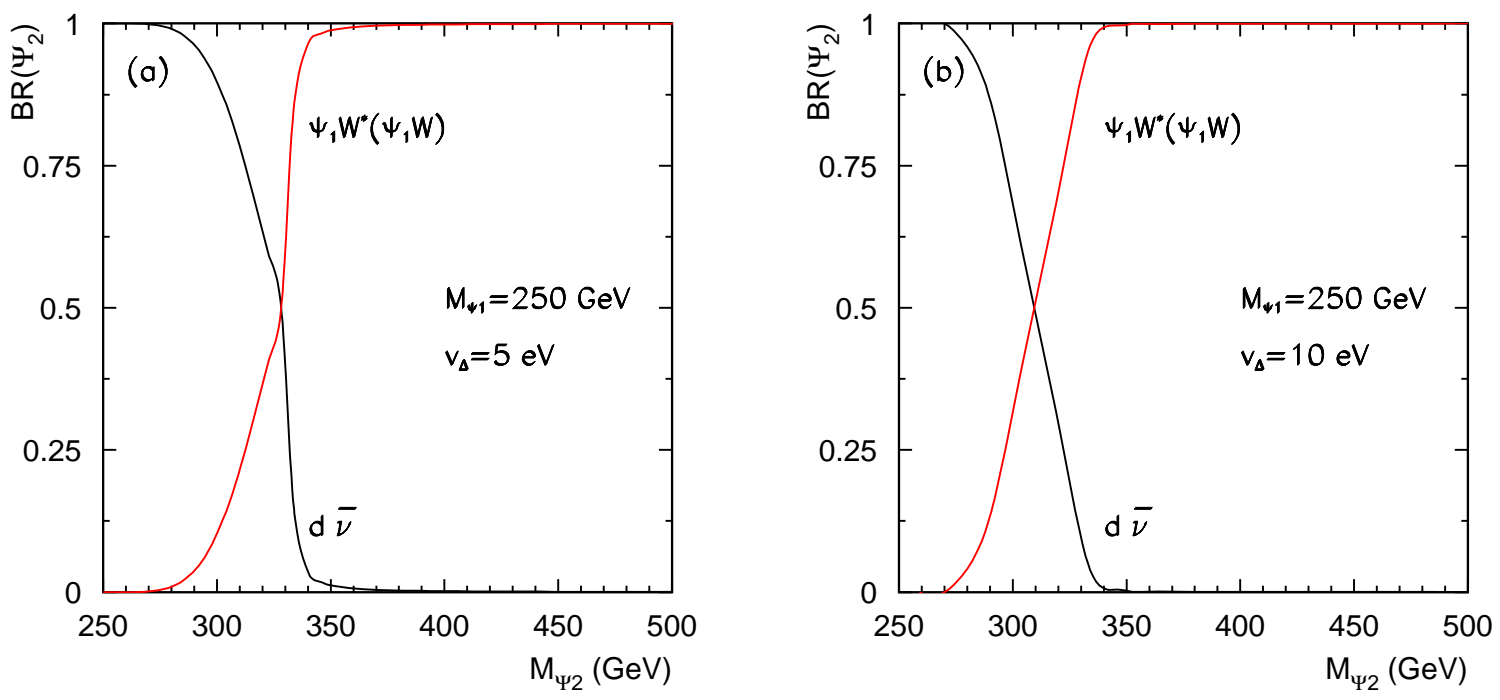

FIG. 9: Branching fractions of $\Psi_{2}$ decay for (a) $v_{\Delta}=5 \mathrm{eV}$ and (b) $10 \mathrm{eV}$, respectively, with $M_{\Psi_{1}}=250 \mathrm{GeV}$.

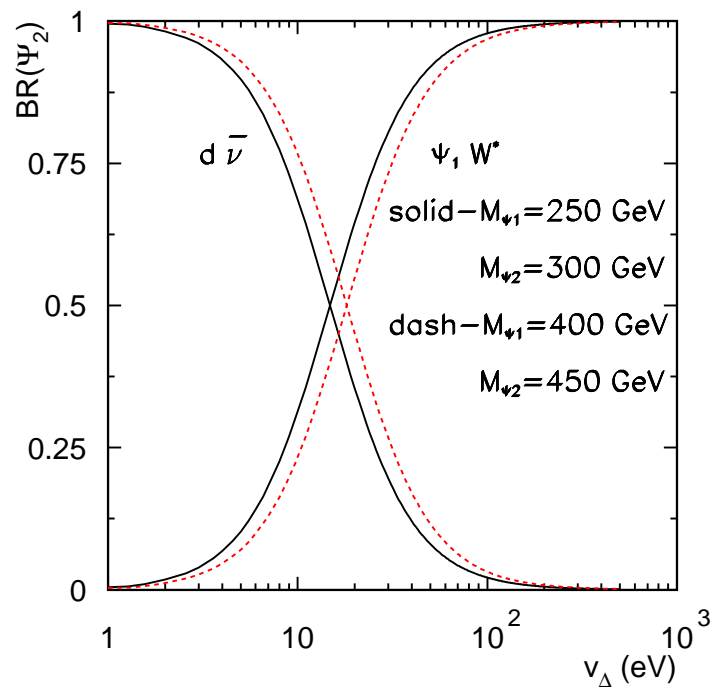

FIG. 10: Branching fractions of $\Psi_{2}$ decay for $M_{\Psi_{1}}=250 \mathrm{GeV}, M_{\Psi_{2}}=300 \mathrm{GeV}$ and $M_{\Psi_{1}}=400 \mathrm{GeV}, M_{\Psi_{2}}=$ $450 \mathrm{GeV}$.

\section{B. Leptoquark Decays and Neutrino Spectra}

As discussed earlier, the lepton-flavor contents of leptoquark decays will be different for each neutrino spectrum. Here, we study this issue in great detail. In Fig. 12,13 and 14 we show the impact of the neutrino 

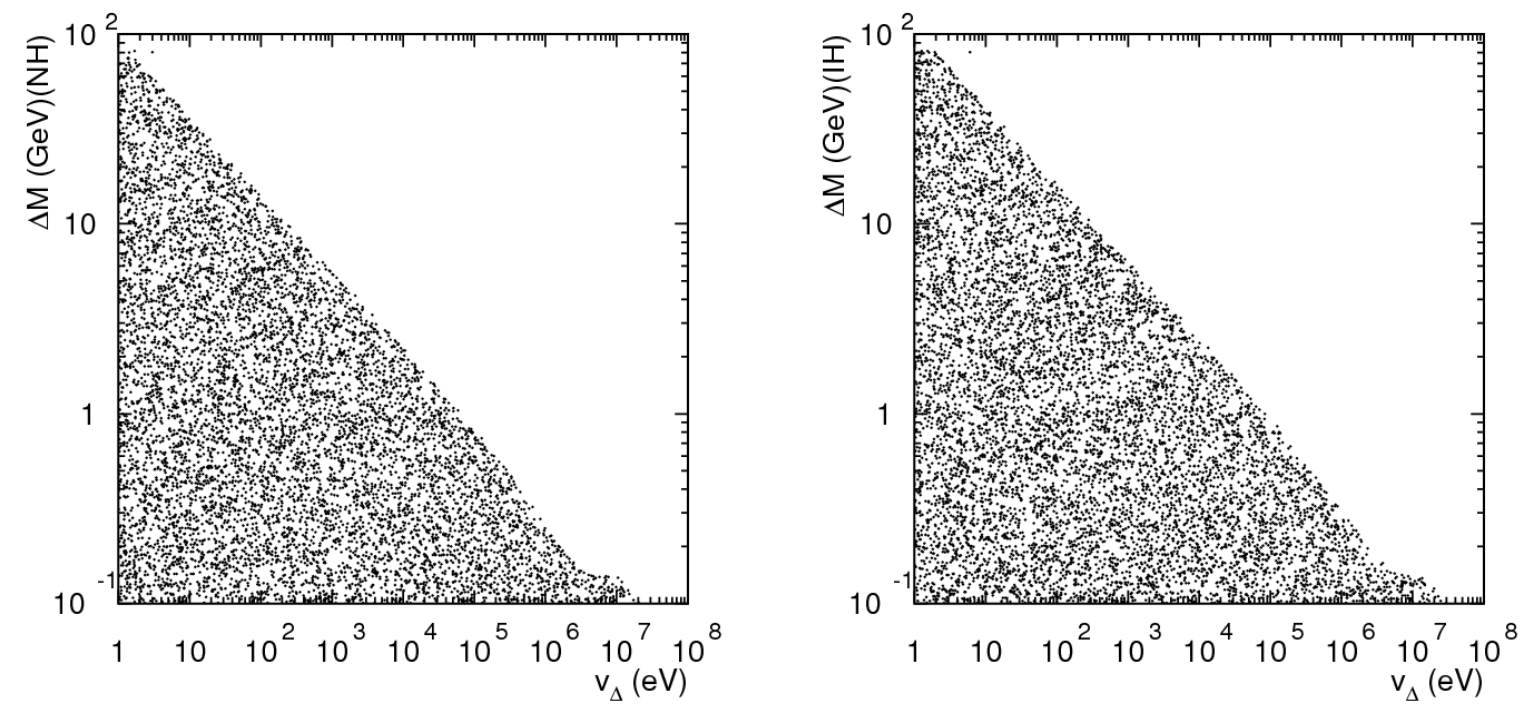

FIG. 11: Allowed region of the parameter space when the leptonic decays of $\Psi_{2}$ are dominant for NH (left) and IH (right). $\Delta M$ versus $v_{\Delta}$ assuming $M_{\Psi_{1}}=250 \mathrm{GeV}$ when $10^{-4} \mathrm{eV} \leq m_{0} \leq 10^{-2} \mathrm{eV}, m_{0}$ is the lightest neutrino mass.

masses and mixing angles on the branching fractions of $\Psi_{1}$ decaying into $e, \mu, \tau$ lepton respectively, with the left panels for the Normal Hierarchy $(\mathrm{NH})$ and the right panels for the Inverted Hierarchy (IH). We first note that the absence of the decay channel be would indicate a $\mathrm{NH}$ with $m_{1}<10^{-2} \mathrm{eV}$, as seen in Fig. 12 In contrast, the be channel is the leading one in the IH. The $s \mu, d \mu$ and $d \tau, s \tau$ channels have the leading branching fractions in the $\mathrm{NH}$, but those channels alone do not seem to provide sufficient information to discriminate among the various possible neutrino mass spectra. If the lightest neutrino mass is above 0.1 $\mathrm{eV}$, then this approaches the "quasi-degenerate" (QD) scenario of the neutrino mass pattern. The channels (be, $s \mu, d \tau$ ) governed by the diagonal neutrino matrix elements reach to about $30 \%$ in this case. The interesting feature here is that $d \mu$ and the $s \tau$ channels vanish due to the unitarity cancelation of the mixing matrix.

The predictions for the decays of $\Psi_{2}$ taking into account the experimental constraints on neutrino mass and mixing angles are shown in Fig. 15. Because of the existence of the missing neutrino in the final state, we must sum over the contributing neutrinos incoherently. As one can see that, in the NH case the decay $\Psi_{2} \rightarrow d \bar{\nu}, s \bar{\nu}$ is the dominant channel, and in the IH case $\Psi_{2} \rightarrow b \bar{\nu}$ is the leading one. In Figs. 16 and 17 we plot the branching fractions of $\Psi_{1}$ the leading decay channels for $\mathrm{NH}$ and $\mathrm{IH}$ versus $M_{\Psi_{1}}$ without any constraints (red stars), and with $K_{L}$ decay constraints and $\mu-e$ conversion constraints (green squares) when $v_{\Delta}=4 \mathrm{eV}$. As noted earlier, present experimental results for these studies strongly constrain the LQ 

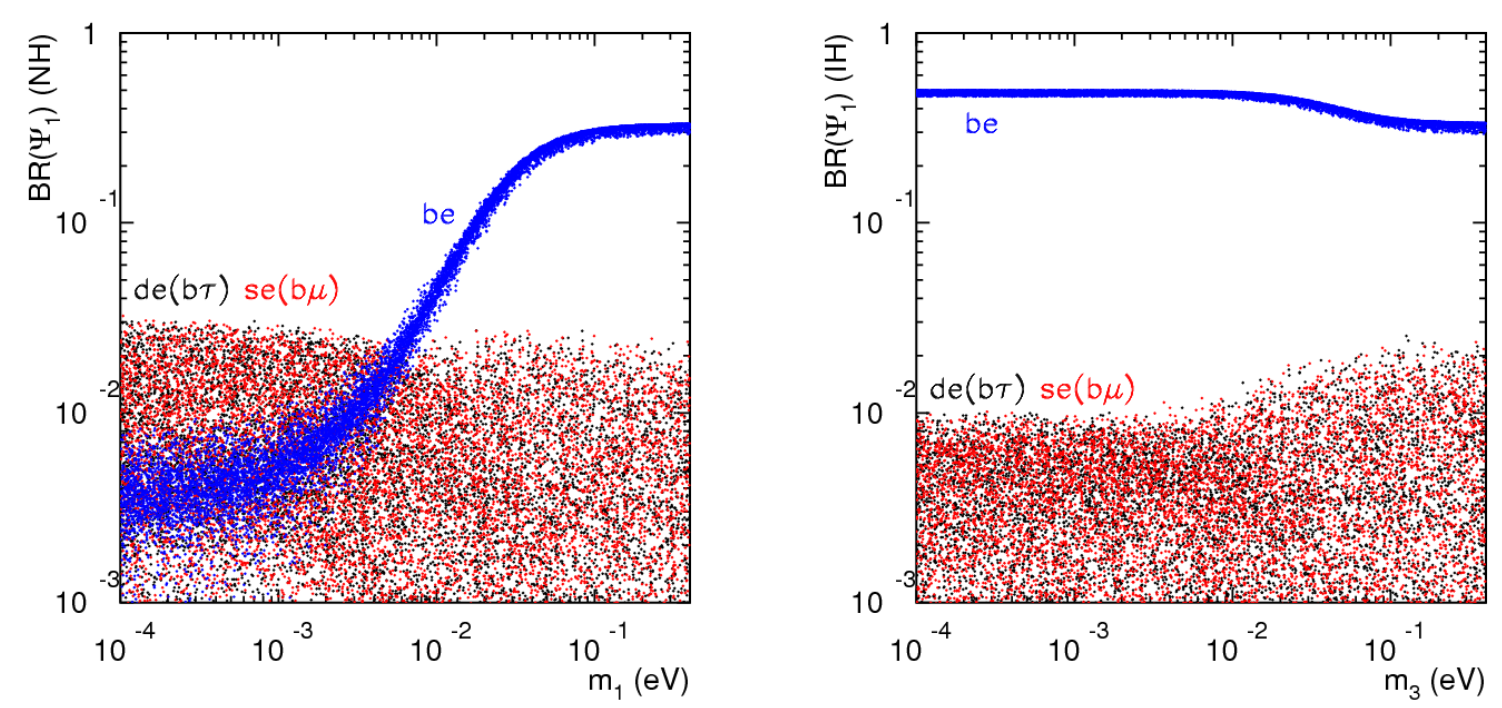

FIG. 12: The branching fractions of $\Psi_{1} \rightarrow d_{i} e\left(d_{i}=d, s, b\right)$ versus the lowest neutrino mass for NH (left) and IH (right) when all the phases vanish. Due to the symmetry as in Eq. (20), the equal channels are also indicated in the parentheses.

parameters, but do not preclude the possibility of scenarios in the LQ is sufficiently light to be discovered at the LHC. In the event of such a discovery, more precise measurements of $B\left(K_{L} \rightarrow \mu^{+} \mu^{-}\right)$and more sensitive searches for $\mu-e$ conversion would provide a consistency test for this model.

\section{Impact of Majorana Phases in Leptoquark Decays}

Although the decays of the leptoquark $\Psi_{1}$ are independent of the phases in the matrices $K_{3}$ and $B$, the unknown Majorana phases could modify the predictions for their decays. In this section we study the predictions for the LQ decays including the impact of the Majorana phases. It is important to note that the $\Psi_{2}$ decay branching ratios are independent of all unknown phases, including the phases in the $K_{3}$ and $B$ matrices and the Majorana phases, after summing over all final state neutrino flavors incoherently. This feature could allow us to probe the neutrino mass spectrum as well as the Majorana phases when combining the information from the $\Psi_{1}$ decays. To illustrate this point, we consider the limiting cases of $m_{1} \approx 0$ $\left(m_{3} \approx 0\right)$ for the NH $(\mathrm{IH})$, an approximation that we expect to hold when the mass of the lightest neutrino in either case is smaller than $\sim 10^{-2} \mathrm{eV}$. For a heavier mass of $m_{1,3}>10^{-1} \mathrm{eV}$, the situation approaches the quasi-degenerate, as we discuss below. 

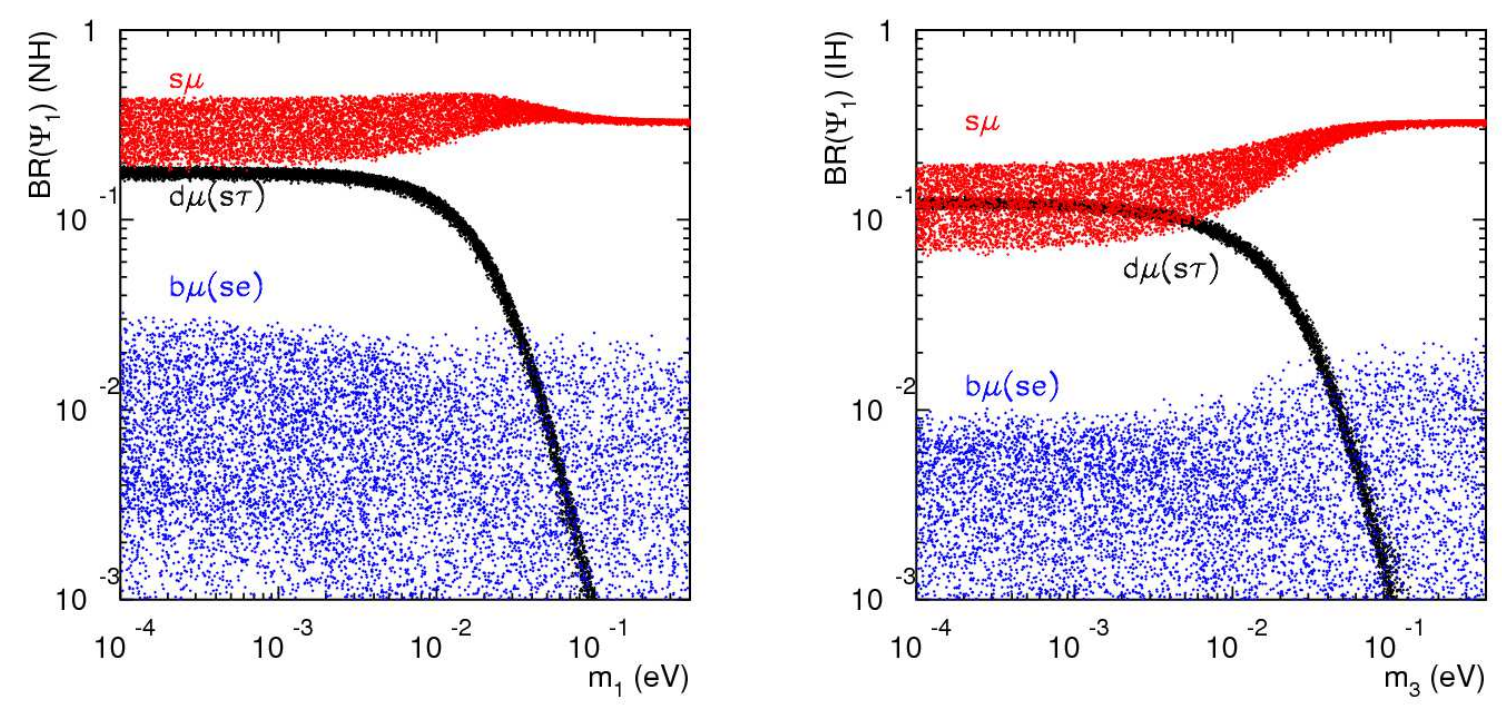

FIG. 13: The branching fractions of $\Psi_{1} \rightarrow d_{i} \mu\left(d_{i}=d, s, b\right)$ versus the lowest neutrino mass for NH (left) and IH (right) when all the phases vanish. Due to the symmetry as in Eq. (20), the equal channels are also indicated in the parentheses.

\section{Normal Hierarchy with one massless neutrino $\left(m_{1} \approx 0\right)$}

The leptoquark decay rates depend on only one Majorana phase $\Phi_{2}$ when $m_{1} \approx 0$ in the NH case. Taking $s_{13}=0$ for simplicity, one finds the expressions for the physical couplings:

$$
\begin{aligned}
e^{-i \beta_{3}} \Gamma_{1}^{31} & =\frac{1}{v_{\Delta}} e^{-2 i \alpha_{1}}\left(\sqrt{\Delta m_{21}^{2}} s_{12}^{2}\right) \\
e^{-i \beta_{2}} \Gamma_{1}^{21} & =e^{-i \beta_{3}} \Gamma_{1}^{32}=\frac{1}{v_{\Delta}} e^{-i\left(\alpha_{1}+\alpha_{2}\right)}\left(\sqrt{\Delta m_{21}^{2}} s_{12} c_{12} c_{23}\right), \\
e^{-i \beta_{1}} \Gamma_{1}^{11} & =e^{-i \beta_{3}} \Gamma_{1}^{33}=\frac{1}{v_{\Delta}} e^{-i\left(\alpha_{1}+\alpha_{3}\right)}\left(-\sqrt{\Delta m_{21}^{2}} s_{12} c_{12} s_{23}\right), \\
e^{-i \beta_{2}} \Gamma_{1}^{22} & =\frac{1}{v_{\Delta}} e^{-2 i \alpha_{2}}\left(\sqrt{\Delta m_{21}^{2}} c_{12}^{2} c_{23}^{2}+\sqrt{\Delta m_{31}^{2}} e^{-i \Phi_{2}} s_{23}^{2}\right) \approx \sqrt{\frac{\Delta m_{31}^{2}}{v_{\Delta}^{2}}} e^{-i\left(2 \alpha_{2}+\Phi_{2}\right)} s_{23}^{2}, \\
e^{-i \beta_{1}} \Gamma_{1}^{12} & =e^{-i \beta_{2}} \Gamma_{1}^{23}=\frac{1}{v_{\Delta}} e^{-i\left(\alpha_{2}+\alpha_{3}\right)}\left(-\sqrt{\Delta m_{21}^{2}} c_{12}^{2}+\sqrt{\Delta m_{31}^{2}} e^{-i \Phi_{2}}\right) s_{23} c_{23} \\
& \approx \sqrt{\frac{\Delta m_{31}^{2}}{v_{\Delta}^{2}}} e^{-i\left(\alpha_{2}+\alpha_{3}+\Phi_{2}\right)} s_{23} c_{23},
\end{aligned}
$$

and

$$
e^{-i \beta_{1}} \Gamma_{1}^{13}=\frac{1}{v_{\Delta}} e^{-2 i \alpha_{3}}\left(\sqrt{\Delta m_{21}^{2}} c_{12}^{2} s_{23}^{2}+\sqrt{\Delta m_{31}^{2}} e^{-i \Phi_{2}} c_{23}^{2}\right) \approx \sqrt{\frac{\Delta m_{31}^{2}}{v_{\Delta}^{2}}} e^{-i\left(2 \alpha_{3}+\Phi_{2}\right)} c_{23}^{2} .
$$



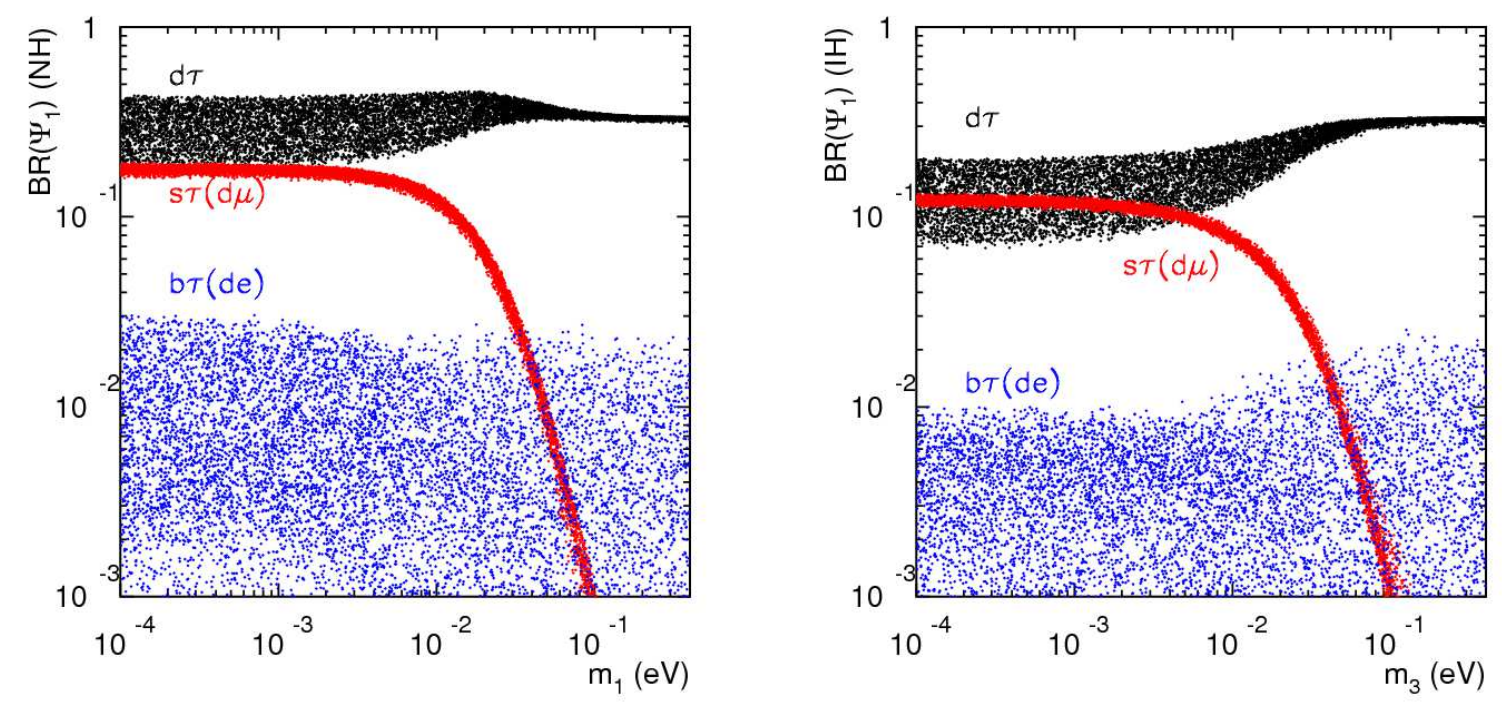

FIG. 14: The branching fractions of $\Psi_{1} \rightarrow d_{i} \tau\left(d_{i}=d, s, b\right)$ versus the lowest neutrino mass for NH (left) and IH (right) when all the phases vanish. Due to the symmetry as in Eq. (20), the equal channels are also indicated in the parentheses.

The behavior of the branching fractions for the dominant channels is shown in Fig. 18. We can see the rather weak dependence of the decay branching fractions on the phase $\Phi_{2}$ that can be also understood from the above analytical expressions. When the phase $\Phi_{2}=\pi$, one obtains the maximal suppression (enhancement) for the channels $\Psi_{1} \rightarrow s \mu^{+}$and $\Psi_{1} \rightarrow d \tau^{+}\left(\Psi_{1} \rightarrow d \mu^{+}, s \tau^{+}\right)$, by a factor two at most. It is important to note that the braching ratio of the sum over all the quark contributions for each lepton flavor, $s \mu^{+}+d \mu^{+}$ or $d \tau^{+}+s \tau^{+}$, remains the same. Thus, from the observational point of view in collider experiments, the signals of $\mu+$ jet and $\tau+$ jet will be unchanged, insensitive to the Majorana phase.

\section{Inverted Hierarchy with one massless neutrino $\left(m_{3} \approx 0\right)$}

In the case of the Inverted Hierarchy with one massless neutrino one finds that all relevant decays depend on only one phase $\Phi_{1}$. The relevant couplings for $s_{13}=0$ are given by

$$
\begin{aligned}
e^{-i \beta_{3}} \Gamma_{1}^{31} & =\frac{1}{v_{\Delta}} e^{-2 i \alpha_{1}}\left(\sqrt{\Delta m_{21}^{2}+\left|\Delta m_{31}^{2}\right|} s_{12}^{2}+\sqrt{\left|\Delta m_{31}^{2}\right|} e^{-i \Phi_{1}} c_{12}^{2}\right) \\
& \approx \sqrt{\frac{\left|\Delta m_{31}^{2}\right|}{v_{\Delta}^{2}}} e^{-2 i \alpha_{1}}\left(s_{12}^{2}+e^{-i \Phi_{1}} c_{12}^{2}\right),
\end{aligned}
$$



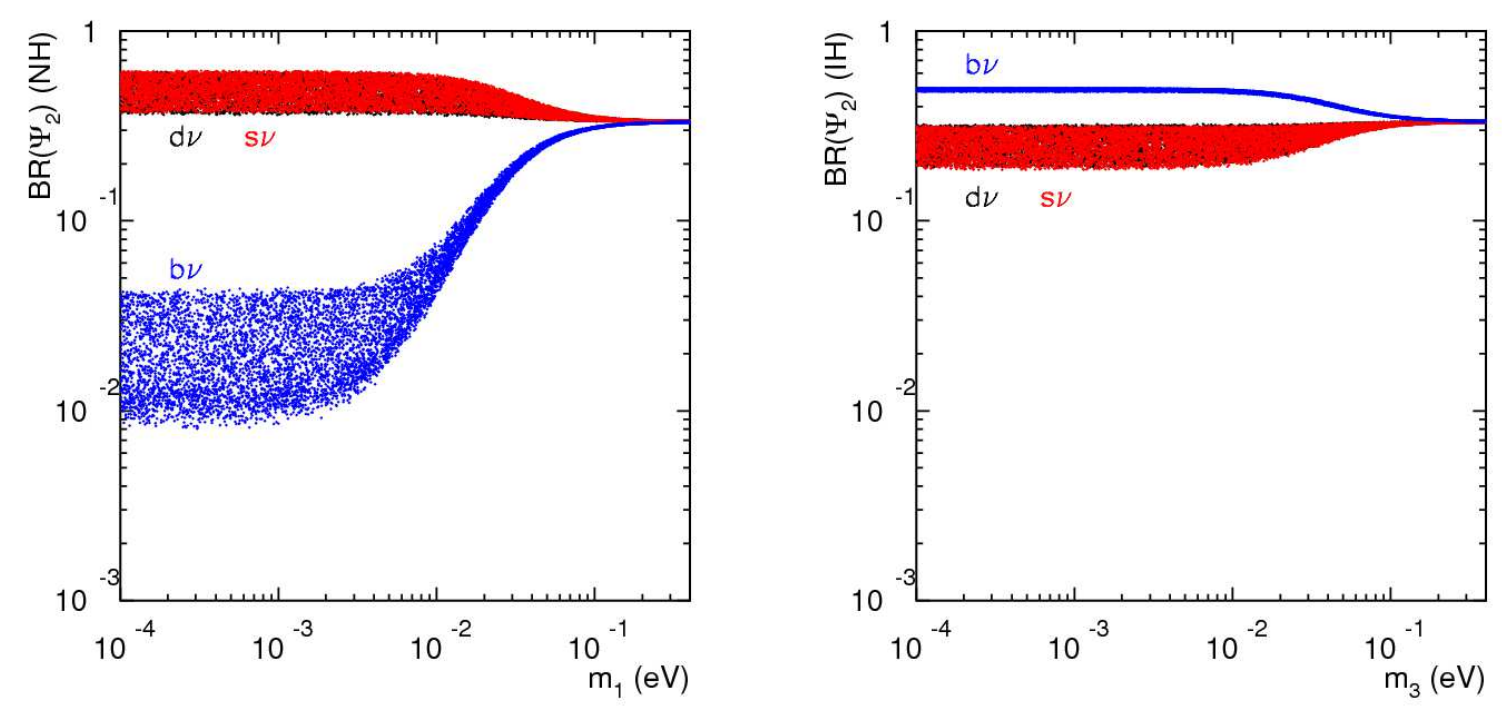

FIG. 15: $\Psi_{2}$ branching fractions versus the lowest neutrino mass for NH (left) and IH (right), independent of the phases.

$$
\begin{aligned}
& e^{-i \beta_{2}} \Gamma_{1}^{21}=e^{-i \beta_{3}} \Gamma_{1}^{32}=\frac{1}{v_{\Delta}} e^{-i\left(\alpha_{1}+\alpha_{2}\right)}\left(\sqrt{\Delta m_{21}^{2}+\left|\Delta m_{31}^{2}\right|}-\sqrt{\left|\Delta m_{31}^{2}\right|} e^{-i \Phi_{1}}\right) c_{12} c_{23} s_{12} \\
& \approx \sqrt{\frac{\left|\Delta m_{31}^{2}\right|}{v_{\Delta}^{2}}} e^{-i\left(\alpha_{1}+\alpha_{2}\right)}\left(1-e^{-i \Phi_{1}}\right) c_{12} c_{23} s_{12}, \\
& e^{-i \beta_{1}} \Gamma_{1}^{11}=e^{-i \beta_{3}} \Gamma_{1}^{33}=\frac{1}{v_{\Delta}} e^{-i\left(\alpha_{1}+\alpha_{3}\right)}\left(-\sqrt{\Delta m_{21}^{2}+\left|\Delta m_{31}^{2}\right|}+\sqrt{\left|\Delta m_{31}^{2}\right|} e^{-i \Phi_{1}}\right) c_{12} s_{23} s_{12} \\
& \approx \sqrt{\frac{\left|\Delta m_{31}^{2}\right|}{v_{\Delta}^{2}}} e^{-i\left(\alpha_{1}+\alpha_{3}\right)}\left(-1+e^{-i \Phi_{1}}\right) c_{12} s_{23} s_{12}, \\
& e^{-i \beta_{2}} \Gamma_{1}^{22}=\frac{1}{v_{\Delta}} e^{-2 i \alpha_{2}}\left(\sqrt{\Delta m_{21}^{2}+\left|\Delta m_{31}^{2}\right|} c_{12}^{2}+\sqrt{\left|\Delta m_{31}^{2}\right|} e^{-i \Phi_{1}} s_{12}^{2}\right) c_{23}^{2} \\
& \approx \sqrt{\frac{\left|\Delta m_{31}^{2}\right|}{v_{\Delta}^{2}}} e^{-2 i \alpha_{2}}\left(c_{12}^{2}+e^{-i \Phi_{1}} s_{12}^{2}\right) c_{23}^{2}, \\
& e^{-i \beta_{1}} \Gamma_{1}^{12}=e^{-i \beta_{2}} \Gamma_{1}^{23}=\frac{1}{v_{\Delta}} e^{-i\left(\alpha_{2}+\alpha_{3}\right)}\left(-\sqrt{\Delta m_{21}^{2}+\left|\Delta m_{31}^{2}\right|} c_{12}^{2}-\sqrt{\left|\Delta m_{31}^{2}\right|} e^{-i \Phi_{1}} s_{12}^{2}\right) s_{23} c_{23} \\
& \approx \sqrt{\frac{\left|\Delta m_{31}^{2}\right|}{v_{\Delta}^{2}}} e^{-i\left(\alpha_{2}+\alpha_{3}\right)}\left(-c_{12}^{2}-e^{-i \Phi_{1}} s_{12}^{2}\right) s_{23} c_{23},
\end{aligned}
$$

and

$$
\begin{aligned}
e^{-i \beta_{1}} \Gamma_{1}^{13} & =\frac{1}{v_{\Delta}} e^{-2 i \alpha_{3}}\left(\sqrt{\Delta m_{21}^{2}+\left|\Delta m_{31}^{2}\right|} c_{12}^{2}+\sqrt{\left|\Delta m_{31}^{2}\right|} e^{-i \Phi_{1}} s_{12}^{2}\right) s_{23}^{2} \\
& \approx \sqrt{\frac{\left|\Delta m_{31}^{2}\right|}{v_{\Delta}^{2}}} e^{-2 i \alpha_{3}}\left(c_{12}^{2}+e^{-i \Phi_{1}} s_{12}^{2}\right) s_{23}^{2} .
\end{aligned}
$$



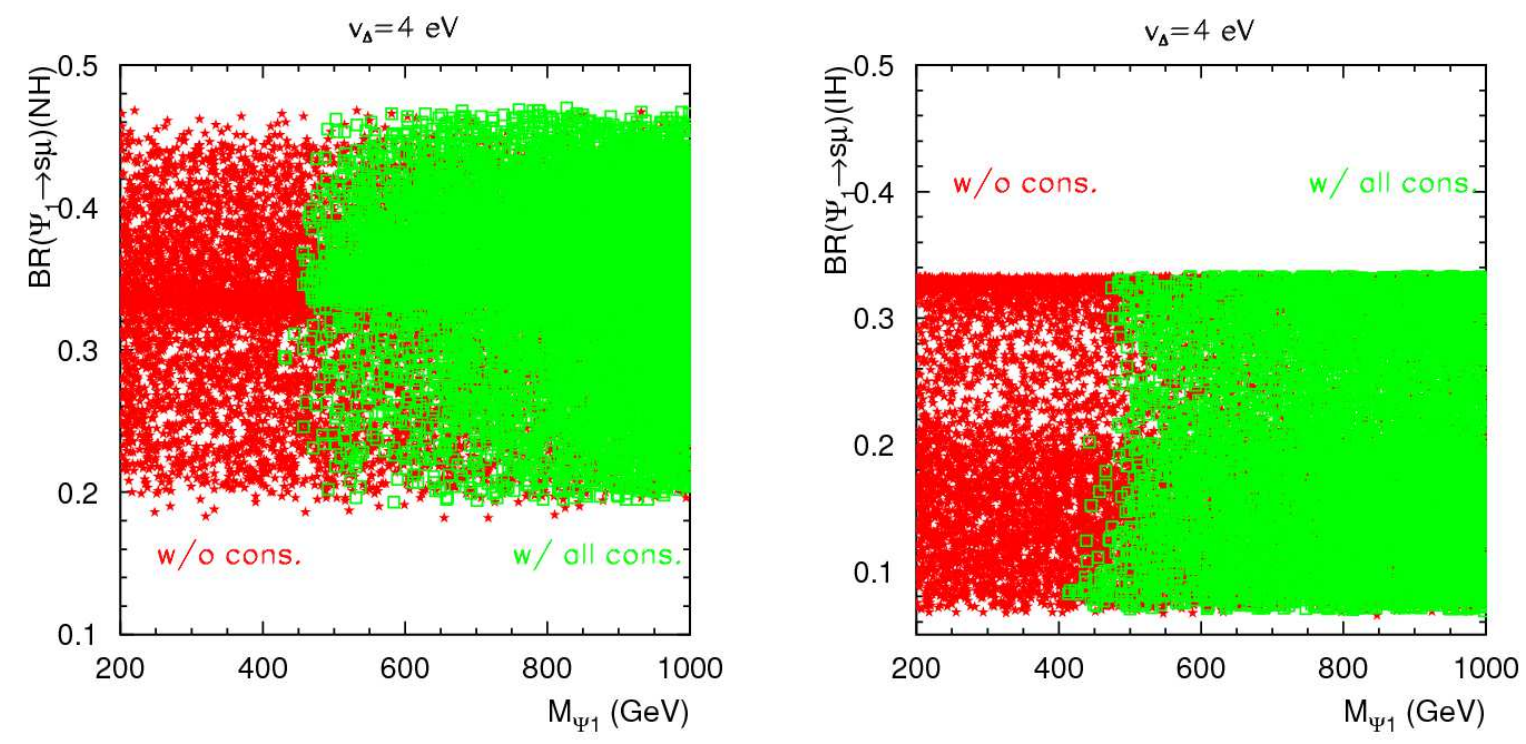

FIG. 16: The branching fraction of leading channel $\Psi_{1} \rightarrow s \mu$ versus leptoquark mass for NH (left) and IH (right) without any constraints (red solid star), and with both $K_{L}$ decay and $\mu-e$ conversion constraints (green empty square), $v_{\Delta}=4 \mathrm{eV}$.

In Fig. 19 we show the dependence of the branching fractions on this Majorana phase. The maximal suppression or enhancement takes place also when $\Phi_{1}=\pi$. In this scenario the dominant channels also swap from one to the other. Unlike in the case of the NH where $s \mu \leftrightarrow d \mu$ and $s \tau \leftrightarrow d \tau$ at $\Phi_{2}=\pi$, the interchanges of the channels occur for $s \mu, d \mu \leftrightarrow b \mu$, and $d \tau, s \tau \leftrightarrow b \tau$, as well as be $\leftrightarrow d e, s e$, when $\Phi_{1}$ varies from 0 to $\pi$. Once again, we notice that the branching ratio for the sum over all the quark contributions for each lepton flavor, $b e^{+}+d e^{+}+s e^{+}$or $s \mu^{+}+d \mu^{+}+b \mu^{+}$or $d \tau^{+}+s \tau^{+}+b \tau^{+}$remains the same. This fact makes the collider search for a leptonic channel rather phase-independent. However, if we require a $b$-flavor tagging in the event selection along with the lepton flavor identification, this could provide crucial information on the value of the Majorana phase $\Phi_{1}$. Specifically, we see the qualitative features in the IH

$$
\operatorname{BR}(b e) \begin{cases}\gg \operatorname{BR}(b \mu), \operatorname{BR}(b \tau) & \text { for } \Phi_{1} \approx 0 \\ \ll \operatorname{BR}(b \mu), \operatorname{BR}(b \tau) & \text { for } \Phi_{1} \approx \pi .\end{cases}
$$

\section{Quasi-Degenerate Hierarchy}

For a QD neutrino spectrum, the approximation of a nearly massless lightest neutrino no longer applies, and one must use the full expressions for the $\Gamma^{i j}$ that are given in Appendix $\mathrm{A}$. As one can readily appreciate from these expressions by setting $m_{1} \approx m_{2} \approx m_{3}$, the branching ratios for the $\Psi_{1}$ will in general depend on 

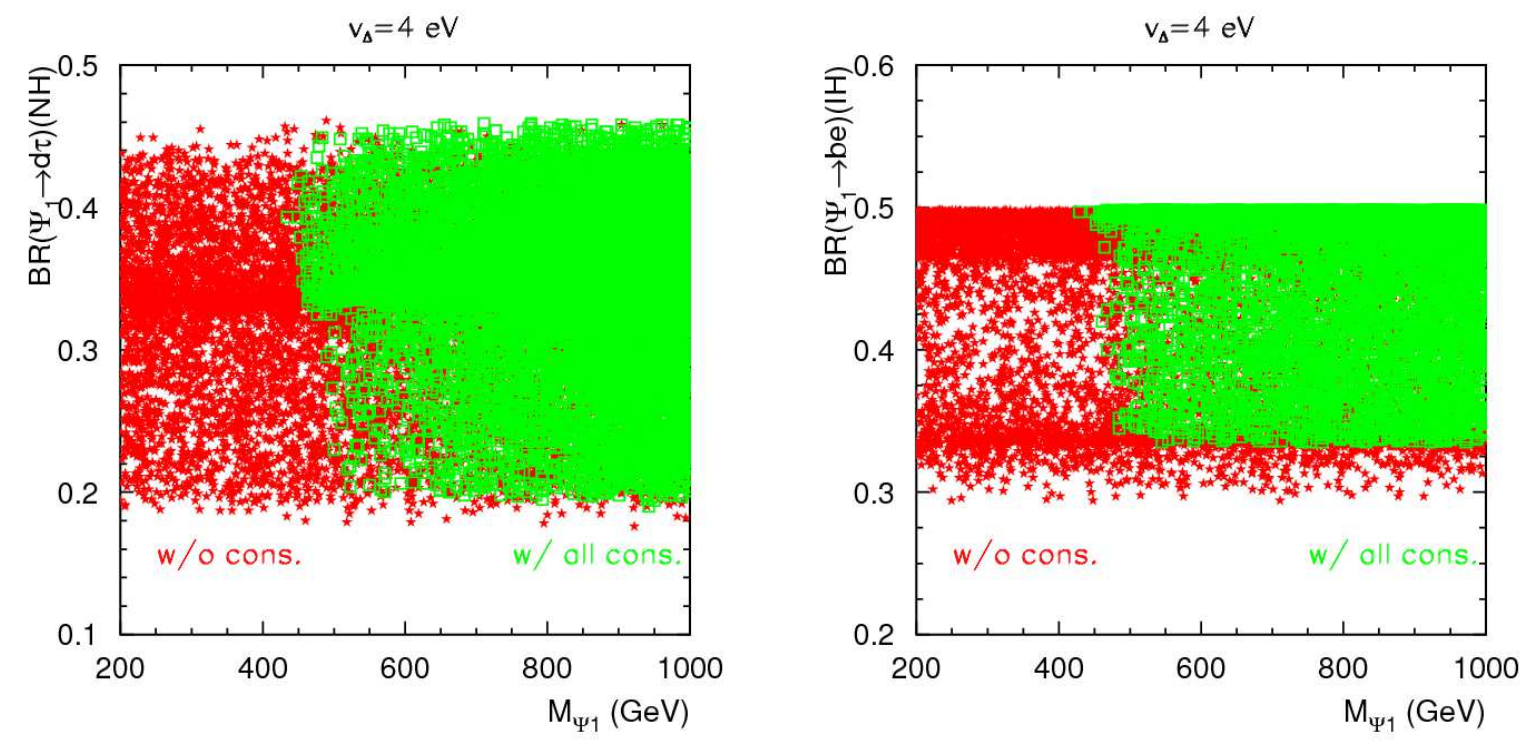

FIG. 17: The branching fraction of leading channel $\Psi_{1} \rightarrow d \tau$ for NH (left) and $\Psi_{1} \rightarrow$ be for IH (right) versus leptoquark mass without any constraints (red solid star), and with both $K_{L}$ decay and $\mu-e$ conversion constraints (green empty square), $v_{\Delta}=4 \mathrm{eV}$.

both $\Phi_{1}$ and $\Phi_{2}$. From a numerical scan over the neutrino masses and mixing angles, we find that the mild $\Phi_{2}$-dependences of the branching ratios for $\Phi_{1}=0$ are quite similar to those of the $\mathrm{NH}$, and that the strong $\Phi_{1}$-dependences of the branching ratios for $\Phi_{2}=0$ are quite similar to those of the IH. Again, if $b$-flavor tagging is effective along with the lepton flavor identification, one could hope to probe crucial information on the value of the Majorana phase $\Phi_{1}$. The only difference from the IH case is the numerical values for the branching ratio of the leading channels.

\section{Total Decay Width of Leptoquarks}

To complete our study about the LQ properties, in Fig. 20 we plot the total width (left axis) and decay length (right axis) for $\Psi_{1}$ versus $v_{\Delta}$ for $M_{\Psi_{1}}=250 \mathrm{GeV}$ in $\mathrm{NH}$ and $\mathrm{IH}$. The total decay width is proportional to $M_{\Psi_{1}} / v_{\Delta}^{2}$. At the values $v_{\Delta}<10^{4} \mathrm{eV}$, its decay is prompt. This is the standard scenario for collider searches to be discussed in the next section. For larger values of $v_{\Delta}$, the leptoquark can be long-lived in the detector's scale, making the collider signatures rather different. One may need to search for exotic hadrons or heavy charged tracks. Since these very large values of $v_{\Delta}$ are much higher than the light neutrino mass scale for our consideration of neutrino mass generation, we will not pursue this parameter region further. 

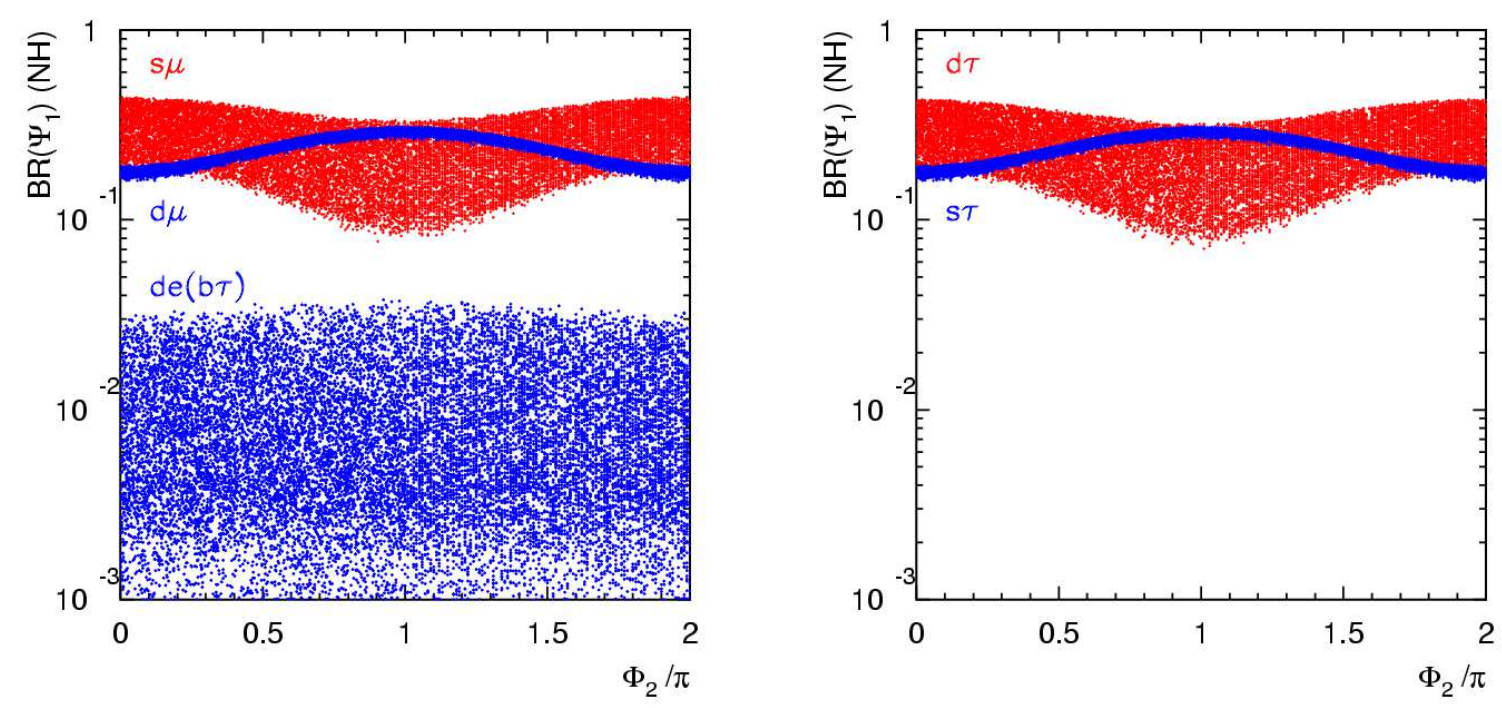

FIG. 18: $\Psi_{1}$ branching fractions versus the Majorana phase $\Phi_{2}$ for the $m_{1}=0$ scenario.

\section{SEARCH FOR LEPTOQUARKS AT THE LHC}

In this section we study the main production mechanisms of leptoquarks and their experimental signatures at the LHC. There exists extensive literature on this topic, treating both theoretical and phenomenological considerations [25, 26, 27, 28] and as well as experimental searches [29, 30]. Our approach here goes beyond the existing studies in two respects: first we predict specific correlations between observables involving different final state lepton flavors, and second we delineate the connections between these correlations and the light neutrino mixing angles and possible mass spectra.

As is well known in the case of the leptoquarks, the leading production channel is via the QCD interaction

$$
\begin{aligned}
& q+\bar{q} \rightarrow L Q+\overline{L Q} \\
& g+g \rightarrow L Q+\overline{L Q} .
\end{aligned}
$$

The pair production total cross section versus its mass at the LHC is plotted in Fig. 21 (the solid curve). The other unique channel is the single production via the Yukawa interaction

$$
g+q(\bar{q}) \rightarrow L Q(\overline{L Q})+\bar{\ell}(\ell)
$$

This cross section is rather small in our model due to the constraints from the neutrino masses, as we will discuss later. More importantly, the nature of the $S U(2)_{L}$ doublet $\Phi_{b}$ allows for associated production of 

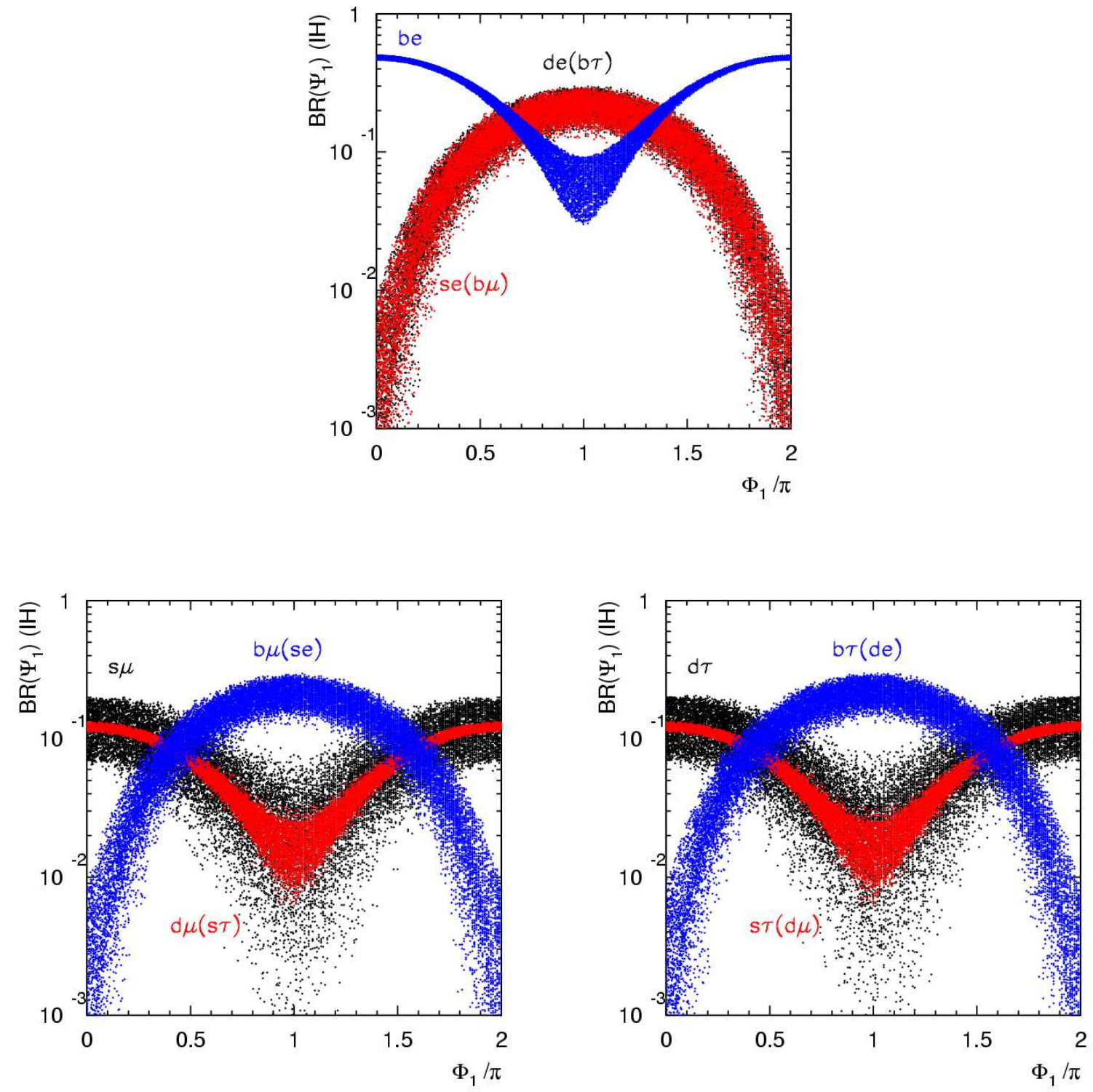

FIG. 19: $\Psi_{1}$ branching fractions versus the Majorana phase $\Phi_{1}$ for the $m_{3}=0$ scenario and $\Phi_{1} \in(0,2 \pi)$.

$\Psi_{1}$ and $\Psi_{2}$ via $W$ exchange,

$$
q\left(p_{1}\right)+\bar{q}^{\prime}\left(p_{2}\right) \rightarrow \Psi_{1}\left(k_{1}\right)+\Psi_{2}^{*}\left(k_{2}\right) .
$$

In terms of the polar angle variable $y=\hat{p}_{1} \cdot \hat{k}_{1}$ in the parton c.m. frame with energy $\sqrt{s}$, the parton level cross section for the associated production is

$$
\frac{d \sigma}{d y}\left(q \bar{q}^{\prime} \rightarrow \Psi_{1} \Psi_{2}^{*}\right)=\frac{\pi \alpha^{2} \beta^{3}\left(1-y^{2}\right)}{32 N_{c} \sin ^{4} \theta_{W}} \frac{s}{\left(s-M_{W}^{2}\right)^{2}},
$$

where $\beta=\sqrt{\left(1-\left(m_{i}+m_{j}\right)^{2} / s\right)\left(1-\left(m_{i}-m_{j}\right)^{2} / s\right)}$ is the speed factor of $\Psi_{1}$ and $\Psi_{2}$ in the c.m. frame. 


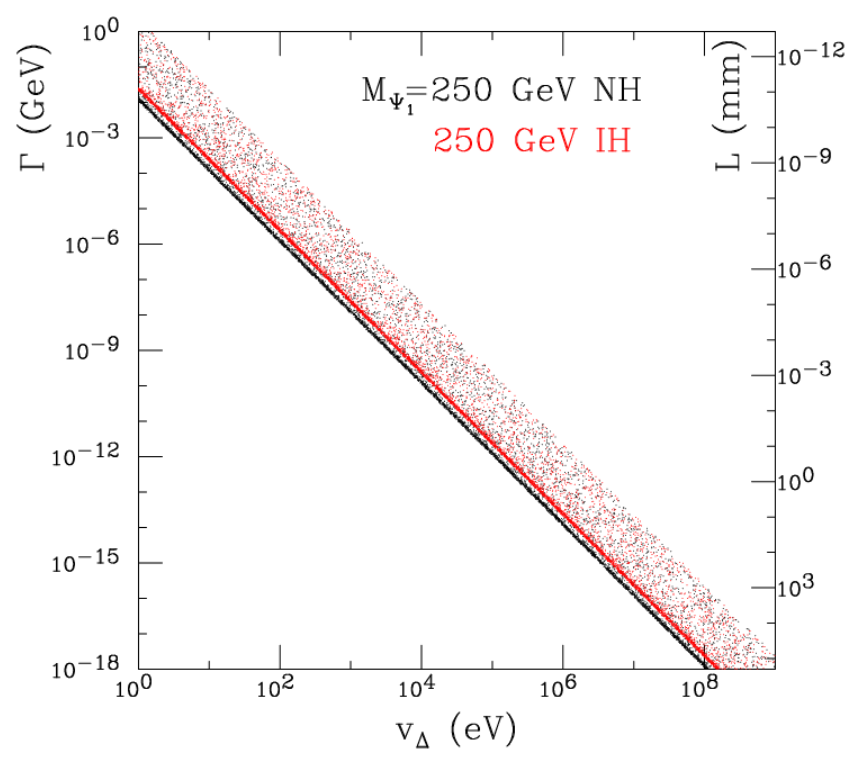

FIG. 20: Total decay width and length of $\Psi_{1}$ versus $v_{\Delta}$.

The total cross section versus its mass at the LHC is plotted in Fig. 21 (the dotted curve), assuming $M_{\Psi_{1}}=$ $M_{\Psi_{2}}$. As expected, the cross section for $\Psi_{1} \Psi_{2}^{*}$ production is smaller than the QCD pair production $\Psi_{1} \Psi_{1}^{*}$ or $\Psi_{2} \Psi_{2}^{*}$ by about three orders of magnitude.

We now turn to the signal observability at the LHC. In Sec. A and B, we are mainly concerned with the kinematical features for the signal and backgrounds. We will take the decay branching fractions of $\Psi_{1}, \Psi_{2}$ to be $100 \%$ to the corresponding channels under discussion. In Sec. C, we will devote ourself to the determination for the branching fractions.

\section{A. Pair Production of $\Psi_{1}$}

The QCD corrections to leptoquark pair production at the LHC have been studied [26] and a NLO Kfactor of order $1.5-1.9$ for leptoquark mass range from $200 \mathrm{GeV}$ to $1500 \mathrm{GeV}$ is predicted. We apply the K-factor 1.5 to the processes in our numerical analysis. Assuming that $\Psi_{1}$ is the lighter leptoquark, its decay modes are

$$
\Psi_{1} \rightarrow d_{i} e_{j}^{+} \quad\left(d_{i}=d, s, b ; \quad e_{j}=e, \mu, \tau\right)
$$

We now explore the signal observability according to the different lepton flavors. 


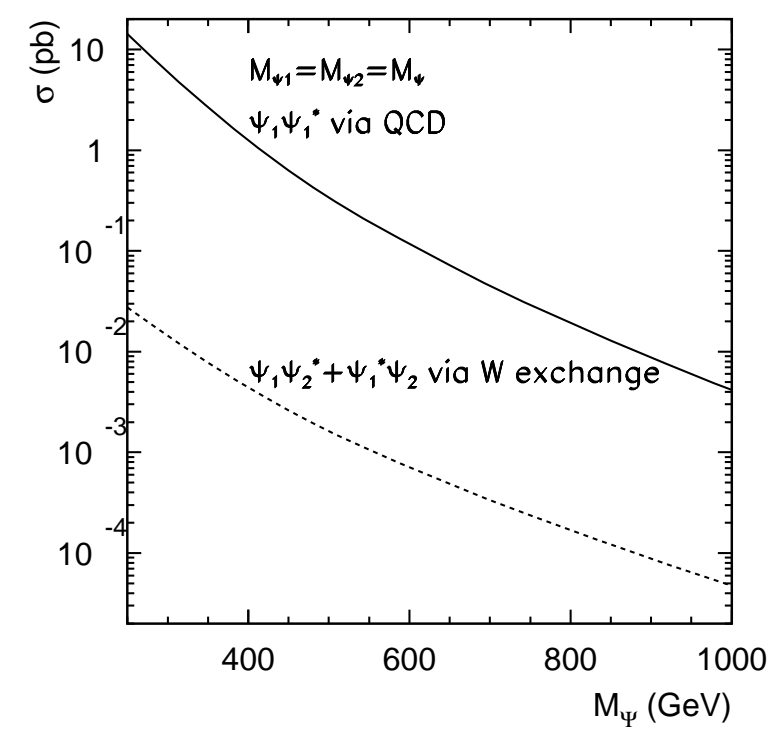

FIG. 21: Leptoquark pair production total cross section at the LHC versus leptoquark mass. The solid curve is for $p p \rightarrow L Q+\overline{L Q}$ via QCD, assuming $\mu_{F}=\mu_{R}=M_{\Psi}=M_{\Psi_{1}}$. The dotted curve is for $p p \rightarrow \Psi_{1} \Psi_{2}^{*}+\Psi_{1}^{*} \Psi_{2}$ via $W^{ \pm}$exchange, assuming $M_{\Psi_{1}}=M_{\Psi_{2}}=M_{\Psi}$.

$$
\text { 1. } \Psi_{1} \Psi_{1}^{*} \rightarrow \ell^{+} \ell^{-} j j \quad(\ell=e, \mu)
$$

We start from the cleanest channels with $e, \mu$ in the final state from $\Psi_{1}$ decay. The signal consists of one pair of opposite-sign leptons of arbitrary $e, \mu$ flavor combinations plus two jets of $d, s, b$ quarks. We employ the following basic acceptance cuts for the event selection [31]

$$
\begin{aligned}
& p_{T}(\ell) \geq 15 \mathrm{GeV},|\eta(\ell)|<2.5, \\
& p_{T}(j) \geq 25 \mathrm{GeV},|\eta(j)|<3.0, \\
& \Delta R_{j j}, \Delta R_{j \ell}, \Delta R_{\ell \ell} \geq 1.0
\end{aligned}
$$

where we require a large $\Delta R$ between the jet and lepton to resolve them because both $\Psi_{1}$ s are produced nearly at rest implying that their two-body decay products are back-to-back. To simulate the detector effects on the energy-momentum measurements, we smear the electromagnetic energy, the electromagnetic energy and jet energy by a Gaussian distribution whose width is parameterized as [31]

$$
\begin{aligned}
& \frac{\Delta E}{E}=\frac{a_{c a l}}{\sqrt{E / \mathrm{GeV}}} \oplus b_{\text {cal }}, \quad a_{\text {cal }}=10 \%, b_{\text {cal }}=0.7 \%, \\
& \frac{\Delta E}{E}=\frac{a_{\text {had }}}{\sqrt{E / \mathrm{GeV}}} \oplus b_{\text {had }}, \quad a_{\text {had }}=50 \%, b_{\text {had }}=3 \% .
\end{aligned}
$$


The leading SM backgrounds to this channel are

$$
Z^{*} / \gamma^{*} j j \rightarrow \ell^{+} \ell^{-} j j, \quad t \bar{t} \rightarrow \ell^{+} \ell^{-} j j+E_{T}
$$

Although the background rates are very large to begin with, the signal and background kinematics are quite different. We outline the characteristics and propose some judicious cuts as follows.

- For a few hundred GeV leptoquark decay, the leptons and jets in final states are very energetic. We tighten up the kinematical cuts

$$
p_{T}^{\max }(\ell)>M_{\Psi_{1}} / 4, \quad p_{T}^{\max }(j)>M_{\Psi_{1}} / 4
$$

- To remove the $j j Z^{*}$ background, we veto the lepton pairs with opposite charges in the $Z$-mass window $\left|M_{\ell^{+} \ell^{-}}-M_{Z}\right|>15 \mathrm{GeV}$. This is a standard cut to remove the on-shell $Z$ contribution.

- To remove the $t \bar{t}$ background, we veto the events with large missing energy from $W$ decay: $E_{T}<$ $25 \mathrm{GeV}$.

- In order to select the correct lepton and jet combination and reconstruct the leptoquark, we take advantage of the feature that the two leptoquarks have equal masses $M_{\ell_{1} j_{1}}=M_{\ell_{2} j_{2}}$. In practice, we take $\left|M_{\ell_{1} j_{1}}-M_{\ell_{2} j_{2}}\right|<M_{\Psi_{1}} / 10$. This helps for the background reduction, in particular for $j j \gamma^{*} / Z^{*}$.

The production cross section for the $\Psi_{1} \Psi_{1}^{*}$ signal with the basic cuts (solid curve) and all of the cuts above (dotted curve) are plotted in Fig. 22 For comparison, the background processes of $j j \gamma^{*} / Z^{*}$ and $t \bar{t}$ are also included with the sequential cuts as indicated. Incidentally, the two background curves after the $Z / W$ veto cuts coincide with each other. The backgrounds are suppressed substantially. There are also other subleading backgrounds, such as $W Z, Z Z$ and $W^{+} W^{-} j j$. Their production rates are much smaller compared to those mentioned above. For instance, including the decay branching fractions, we have [32]

$$
\begin{aligned}
& \sigma(W Z) \sim 17.25 \mathrm{pb} \times \frac{2}{3} \times 6.72 \%=0.77 \mathrm{pb}, \\
& \sigma(Z Z) \sim 6.7 \mathrm{pb} \times 69.9 \% \times 6.72 \%=0.3 \mathrm{pb} \\
& \sigma\left(W^{+} W^{-} j j\right) \sim 0.2 \mathrm{pb} \quad \text { with basic cuts. }
\end{aligned}
$$

Therefore, these backgrounds become negligible after our selective cuts as discussed above.

Finally, when we perform a signal significance analysis, we look for a resonance in the mass distribution of the $\ell j$ pair. The invariant mass of $\ell+j$ is plotted in Fig. 23 (a) for $400 \mathrm{GeV}$ leptoquark production. The 


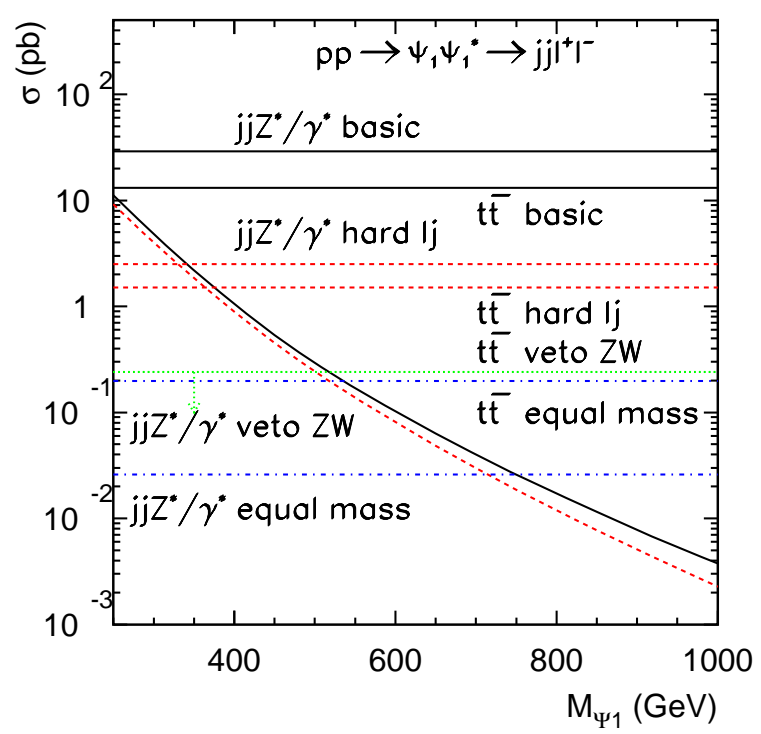

FIG. 22: Production cross section of $\Psi_{1} \Psi_{1}^{*}$ with basic cuts and hard final states cut. Branching fractions for leptoquark decay are not included in this plot. For comparison, the background processes are also included with the sequential cuts as indicated.

width of the distribution $\sim \pm 20 \mathrm{GeV}$ is governed by the detector resolution in our simulation. If we look at a mass window of $\left|M_{\ell_{1} j_{1}, \ell_{2} j_{2}}-M_{\Psi_{1}}\right|<M_{\Psi_{1}} / 20$, the backgrounds will be at a negligible level.

We would like to comment on the $b \bar{b} \ell^{+} \ell^{-}$signal because the $\Psi_{1} \rightarrow e^{+} b$ channel plays an important role in distinguishing different neutrino mass spectra. The b-tagging rate is about $50 \%$ [31]. The signal is still sizable with two b jets tagged.

\section{2. $\Psi_{1} \Psi_{1}^{*} \rightarrow \tau^{+} \ell^{-} j j, \quad \tau^{+} \tau^{-} j j$}

Together with the $e$ and $\mu$ produced in the $\Psi_{1}$ decay, the $\tau$ lepton final state from leptoquark decay can play an important role in distinguishing different neutrino mass patterns. Its identification and reconstruction are different from $e, \mu$ final states because a $\tau$ decays promptly and there will always be missing neutrinos in $\tau$ decay products.

In order to reconstruct the events with $\tau \mathrm{s}$ we note that all the $\tau \mathrm{s}$ produced in the decay of few hundred GeV LQ are highly energetic. The missing momentum will be along the direction of the charged track. We thus assume the momentum of the missing neutrinos to be reconstructed by

$$
\vec{p}(\text { invisible })=\kappa \vec{p}(\text { track })
$$



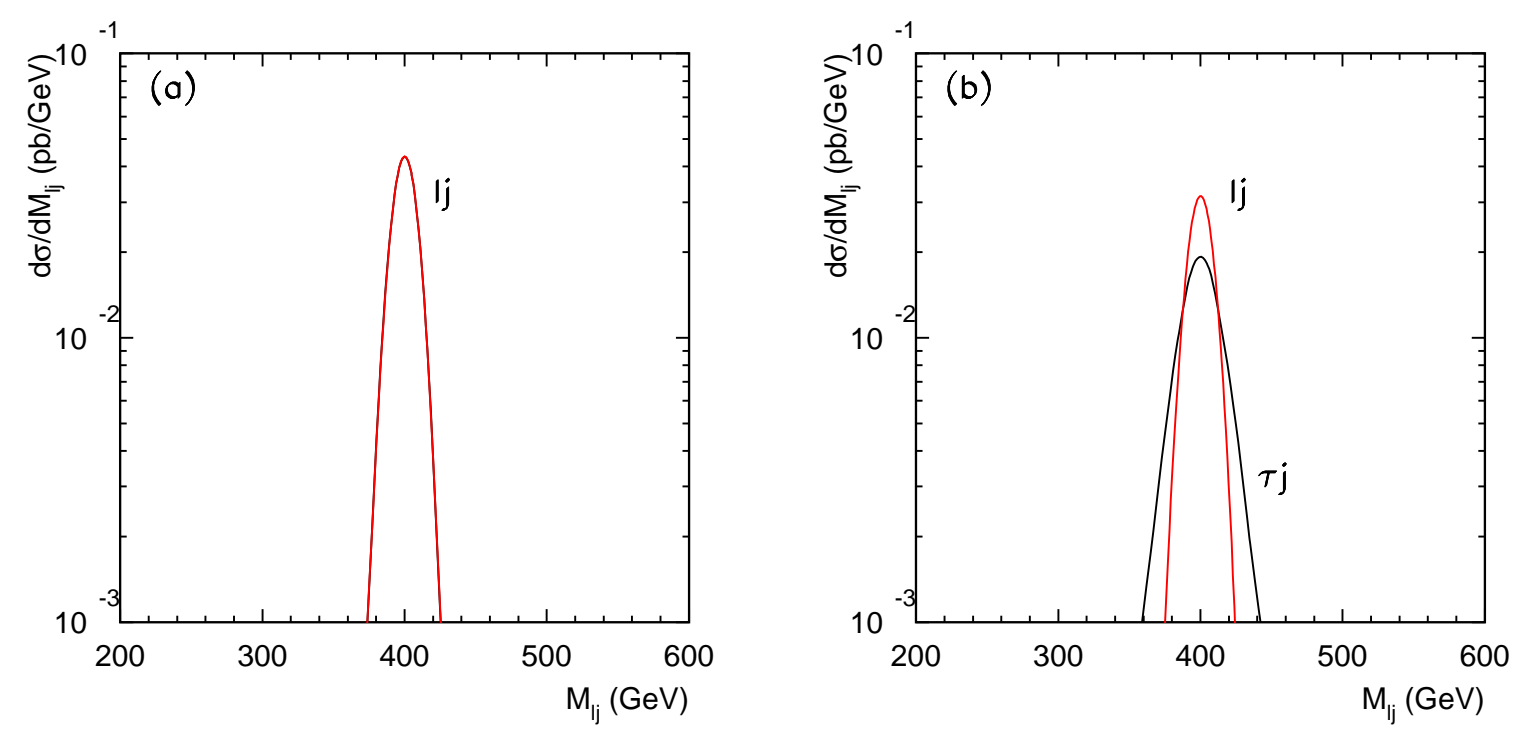

FIG. 23: Reconstructed invariant mass of $M(\ell j)$ for (a) $\ell^{+} \ell^{-} j j$ production and (b) $\ell^{ \pm} \tau^{\mp} j j$ production, with a leptoquark mass of $400 \mathrm{GeV}$.

Identifying $\overrightarrow{p_{T}}$ (invisible) with the measured $\mathscr{E}_{T}$, we thus obtain the $\tau$ momentum by

$$
\vec{p}_{T}(\tau)=\vec{p}_{T}(\ell)+\vec{E}_{T}, \quad p_{L}(\tau)=p_{L}(\ell)+\frac{E_{T}}{p_{T}(\ell)} p_{L}(\ell)
$$

The leptoquark pair kinematics are, thus, fully reconstructed. The reconstructed invariant masses of $M(\ell j)$ and $M(\tau j)$ are plotted in Fig. 23 b). We see that $M(\tau j)$ distribution is slightly broader as anticipated. The rather narrow mass peak of the $\ell j$ system nevertheless serves as the most distinctive kinematical feature for the signal identification.

For $\tau \tau j j$ events with two $\tau$ 's, we generalize the momenta reconstruction to

$$
\vec{p}(\text { invisible })=\kappa_{1} \vec{p}\left(\operatorname{track}_{1}\right)+\kappa_{2} \vec{p}\left(\operatorname{track}_{2}\right)
$$

The proportionality constants $\kappa_{1}, \kappa_{2}$ can be determined from the missing energy measurement as long as the two charge tracks are linearly independent.

In practice when we wish to identify the events with $\tau$ 's, we require a minimal missing transverse energy

$$
E_{T}>20 \mathrm{GeV} \text {. }
$$

This will effectively separate them from the $\ell \ell j j$ events. Another important difference between the leptons from the primary leptoquark decay and from the $\tau$ decay is that latter is much softer. In Fig. 24 we show the $p_{T}$ distribution of the softer lepton from the leptoquark and $\tau$ decays in the events of $\ell \ell j j, \ell \tau j j$ and $\tau \tau j j$, 


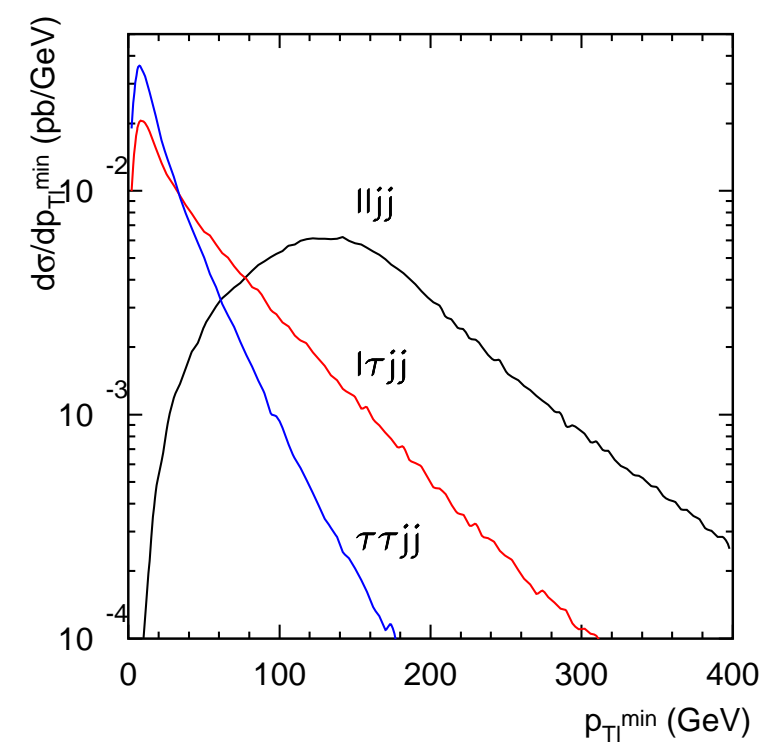

FIG. 24: $p_{T}$ distribution of the softer lepton from the leptoquark and $\tau$ decays in the events of $\ell \ell j j, \ell \tau j j$ and $\tau \tau j j$, for a leptoquark mass $400 \mathrm{GeV}$.

\section{B. Associated Production of $\Psi_{1} \Psi_{2}^{*}\left(\Psi_{1}^{*} \Psi_{2}\right)$}

The total cross section of $\Psi_{1} \Psi_{2}^{*}+\Psi_{1}^{*} \Psi_{2}$ is shown in Fig. 21 by the dotted curve. As discussed earlier, we focus on the fermionic decay $\Psi_{2} \rightarrow d_{i} \nu$. Correspondingly, we only consider the decays $\Psi_{1} \rightarrow d_{i} e$ and $d_{i} \mu$ in order to effectively reconstruct the final state kinematics. The signal is thus

$$
\Psi_{1} \Psi_{2}^{*}, \Psi_{1}^{*} \Psi_{2} \rightarrow \ell^{ \pm} j, \nu j \quad(\ell=e, \mu)
$$

consisting of one charged lepton and two jets plus missing energy. Once again, we consider the corresponding decay branching fractions to be $100 \%$, and will leave the branching fraction determination to the next section.

We employ the following basic acceptance cuts for the event selection

$$
\begin{aligned}
& p_{T}(\ell) \geq 15 \mathrm{GeV},|\eta(\ell)|<2.5, E_{T}>40 \mathrm{GeV} \\
& p_{T}(j) \geq 25 \mathrm{GeV},|\eta(j)|<3.0 \\
& \Delta R_{j j}, \Delta R_{j \ell} \geq 0.4 .
\end{aligned}
$$

To simulate the detector effects on the energy-momentum measurements, we use the same smearing parameters as in the previous section. The irreducible SM backgrounds to this channel are

$$
W^{ \pm} j j, \quad W^{+} W^{-}, W^{ \pm} Z \rightarrow \ell^{ \pm} j j+E_{T} .
$$


The $W j j$ background is by far the largest. To further optimize the signal observability,

- we set additional cuts for hard lepton and jet

$$
p_{T}^{\max }(\ell)>M_{\Psi} / 4, \quad p_{T}^{\max }(j)>M_{\Psi} / 4 .
$$

- The SM background events of $\ell^{ \pm} E_{T}$ typically have the origin from $W^{ \pm}$. We thus veto the $W$ boson with the help of a transverse mass cut $M_{T}(\ell \nu)>150 \mathrm{GeV}$. After this cut, the $W W, W Z$ backgrounds are at a negligible level.

- For the associated production of heavy particles like the two leptoquarks of several hundred $\mathrm{GeV}$, the cluster mass of the system indicates the large threshold. We define

$$
M_{\text {cluster }}=\sqrt{M_{2 j}^{2}+\left(\sum \vec{p}_{T}^{j}\right)^{2}}+\sqrt{\left(\sum \vec{p}_{T}^{\ell}\right)^{2}}+\not_{T}
$$

and will impose a high mass cut to select the signal events $M_{\text {cluster }}>2 M_{\Psi}$.

- The mass reconstruction for $\ell j$ and $\nu j$ can be very indicative. In order to select the correct lepton and jet combination to reconstruct $\Psi_{1}$ we define a transverse mass $M_{T}$ by one jet and the missing transverse energy

$$
M_{T}(j \nu)=\sqrt{\left(E_{T}(j)+E_{T}\right)^{2}-\left(\vec{p}_{T}(j)+\vec{p}_{T}\right)^{2}} .
$$

This variable has an upper bound $M_{\Psi}$, and is typically smaller than $M_{\ell j}$. We thus accept the momentum combination if $M\left(\ell j_{1}\right)>M_{T}\left(j_{2} \nu\right), M\left(\ell j_{2}\right)<M_{T}\left(j_{1} \nu\right)$, or $M\left(\ell j_{2}\right)>M_{T}\left(j_{1} \nu\right), M\left(\ell j_{1}\right)<$ $M_{T}\left(j_{2} \nu\right)$. However, there are two other possibilities that both transverse masses of the two combinations are smaller or larger than their invariant masses. In these cases, we select the combination with smaller $\left|M(\ell j)-M_{T}(j \nu)\right|$ as the right one. In Fig. 25 we show the reconstructed invariant mass and transverse mass distributions for a leptoquark mass $400 \mathrm{GeV}$ for illustration. After selecting correct resonance, we impose transverse mass and invariant mass cuts for leptoquarks

$$
M_{T}(j \nu)<M_{\Psi}, \quad\left|M(\ell j)-M_{\Psi}\right|<20 .
$$

We list the signal and background cross sections with the consecutive cuts in Table. IV] for the case of $M_{\Psi}=400 \mathrm{GeV}$. We see that the $W^{ \pm} j j$ background is at the $O(0.1 \mathrm{fb})$ level. The production cross section of $\Psi_{1} \Psi_{2}^{*}+\Psi_{1}^{*} \Psi_{2}$ versus its mass with basic cuts and all cuts are plotted in Fig. 26 We see that the cuts are highly efficient for the signal and the background processes of $W j j$ is under control up to $M_{\Psi} \sim 1 \mathrm{TeV}$. 


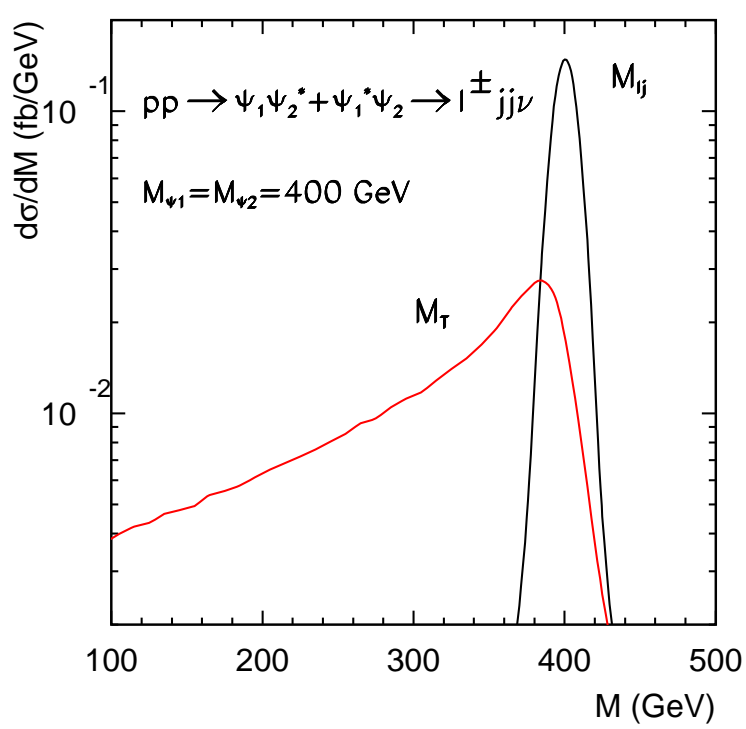

FIG. 25: Reconstructed invariant mass of $M\left(\ell^{ \pm} j\right)$ and transverse mass $M_{T}(j \nu)$ for a leptoquark mass $400 \mathrm{GeV}$.

We would now like to consider the other potentially large backgrounds, namely, $t \bar{t} \rightarrow b b \ell^{+} \ell^{-}+E_{T}$ and leptoquark pair production via QCD $\Psi_{1} \Psi_{1}^{*} \rightarrow j j \ell^{+} \ell^{-}$. Demanding one isolated lepton with the basic cuts and vetoing the extra leptons in the range

$$
p_{T}(\ell)>10 \mathrm{GeV}, \quad|\eta(\ell)|<3.0
$$

the $t \bar{t}$ background is reduced by more than two orders of magnitude and the $\Psi_{1} \Psi_{1}^{*}$ background by about five orders of magnitude. The lepton vetoing is considered part of the basic cuts. With the additional $M_{T}$ and $M(\ell j)$ cuts, the backgrounds would be under control, as listed in Table. IV

\section{Measuring Branching Fractions and Probing the Neutrino Mass Patterns}

So far, we have only studied the characteristic features of the signal and backgrounds for the leading channels and have taken the decay branching fractions of the leptoquarks to be $100 \%$. As we presented earlier, the $\Psi_{1}, \Psi_{2}$ decay branching fractions and the light neutrino mass matrix are directly correlated. Measuring the BR's of different flavor combinations becomes crucial in understanding the neutrino mass pattern and thus the mass generation mechanism. In contrast, it has been customarily assumed [29, 30] that the leptoquark is flavor diagonal in each fermion generation. We list the leading reconstructible leptonic channels along with the predicted branching fractions in Table. $\mathrm{V}$, where the light quarks $(d, s)$ can be 


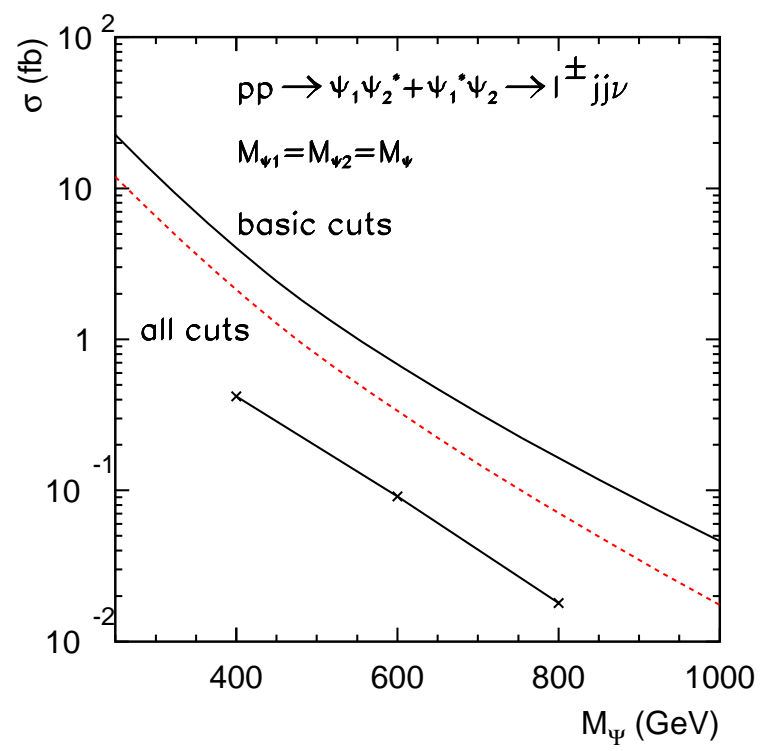

FIG. 26: Total cross section for $\Psi_{1} \Psi_{2}^{*}+\Psi_{1}^{*} \Psi_{2}$ production after the basic cuts (solid curve) and all cuts (dotted curves) and the leading $W j j$ background after all cuts ("×" marks).

\begin{tabular}{|c|c|c|c|c|c|c|}
\hline $\begin{array}{l}\sigma(\mathrm{fb}) \\
\text { cuts }\end{array}$ & $\begin{array}{l}\text { Basic } \\
\text { Cuts }\end{array}$ & $\begin{array}{c}p_{T}^{\ell}, p_{T}^{j} \text { cut } \\
>M_{\Psi} / 4, M_{\Psi} / 4\end{array}$ & $\begin{array}{c}M_{W} \text { veto } \\
M_{T}(\ell \nu)>150 \mathrm{GeV}\end{array}$ & $\begin{array}{l}M_{\text {Cluster }} \\
>2 M_{\Psi}\end{array}$ & $\begin{aligned} & M_{T} \\
< & M_{\Psi}\end{aligned}$ & $\begin{array}{c}M_{\ell j} \\
M_{\Psi} \pm 20 \mathrm{GeV}\end{array}$ \\
\hline $\begin{array}{c}\Psi_{1} \Psi_{2}^{*}+\Psi_{1}^{*} \Psi_{2} \\
M_{\Psi}=400 \mathrm{GeV}\end{array}$ & 3.8 & 3.4 & 2.9 & 2.7 & 2.4 & 2.1 \\
\hline$j j W^{ \pm}$ & & & & & & \\
\hline$M_{\Psi}=400 \mathrm{GeV}$ & $4.8 \times 10^{5}$ & $2.3 \times 10^{4}$ & 9.8 & 9.5 & 8.2 & 0.42 \\
\hline$M_{\Psi}=600 \mathrm{GeV}$ & $4.8 \times 10^{5}$ & 7332 & 6.7 & 5.1 & 4.7 & 0.091 \\
\hline$M_{\Psi}=800 \mathrm{GeV}$ & $4.8 \times 10^{5}$ & 2945 & 4.2 & 2.0 & 1.9 & 0.018 \\
\hline $\begin{array}{c}t \bar{t} \\
M_{\Psi}=400 \mathrm{GeV}\end{array}$ & 186 & 11 & 7.5 & 0.6 & 0.58 & - \\
\hline $\begin{array}{c}\Psi_{1} \Psi_{1}^{*} \\
M_{\Psi}=400 \mathrm{GeV}\end{array}$ & $3.2 \times 10^{-2}$ & $2.4 \times 10^{-2}$ & $7.2 \times 10^{-3}$ & $7.2 \times 10^{-3}$ & $7.1 \times 10^{-3}$ & $5.4 \times 10^{-3}$ \\
\hline
\end{tabular}

TABLE IV: $\Psi_{1} \Psi_{2}^{*}+\Psi_{1}^{*} \Psi_{2}$ signal and irreducible background $j j W^{ \pm}$and reducible backgrounds $t \bar{t}$ and $\Psi_{1} \Psi_{1}^{*}$ for $400 \mathrm{GeV}$ leptoquark mass.

identified as jets, and the $b$ quark may be flavor-tagged. We also associate these channels with predictions of the neutrino mass patterns. We reiterate some of the key observations in connecting the LHC signals of the leptoquarks to the neutrino parameters. 


\begin{tabular}{|c|c|c|c|}
\hline Signal channels & $\begin{array}{l}\text { Leading modes and BR } \\
\text { Normal Hierarchy }\end{array}$ & $\begin{array}{l}\text { Leading modes and BR } \\
\text { Inverted Hierarchy }\end{array}$ & $\begin{array}{c}\text { Leading modes and BR } \\
\text { Quasi degenerate }\end{array}$ \\
\hline \multirow[t]{2}{*}{$\begin{array}{c}\Psi_{1} \Psi_{1}^{*} \\
\Phi_{1}=\Phi_{2}=0\end{array}$} & & $e^{+} b e^{-\bar{b}}: \quad(40-50 \%)^{2}$ & \\
\hline & $\begin{array}{c}\mu^{+} j \approx \tau^{+} j: \\
\text { any } \ell \text { pairs: }(40-60 \%)^{2}\end{array}$ & $\begin{array}{c}\mu^{+} j \approx \tau^{+} j: \\
\text { any } \ell \text { pairs: }(20-30 \%)^{2} \\
\mu^{+} j e^{-} \bar{b} \approx \tau^{+} j e^{-} \bar{b}: \\
(20-30 \%) \times(40-50 \%)\end{array}$ & $\begin{array}{l}e^{+} b \approx \mu^{+} s \approx \tau^{+} d: \\
\text { any } \ell \text { pairs: }(30 \%)^{2}\end{array}$ \\
\hline$\Phi_{1} \approx \pi, \Phi_{2}=0$ & independent of $\Phi_{1}$ & $\begin{array}{c}e b \leftrightarrow e j, \mu j \leftrightarrow \mu b, \tau j \leftrightarrow \tau b \\
e b+e j, \mu j+\mu b, \tau j+\tau b \\
\text { unchanged }\end{array}$ & $\begin{array}{c}e b \leftrightarrow e j, \mu s \leftrightarrow \mu b, \tau d \leftrightarrow \tau b \\
e b+e j, \mu j+\mu b, \tau j+\tau b \\
\text { unchanged }\end{array}$ \\
\hline$\Phi_{1}=0, \Phi_{2} \approx \pi$ & $\begin{array}{c}s \mu \leftrightarrow d \mu, d \tau \leftrightarrow s \tau \\
\mu j, \tau j \text { unchanged }\end{array}$ & independent of $\Phi_{2}$ & $\begin{array}{c}s \mu \leftrightarrow d \mu, d \tau \leftrightarrow s \tau \\
e b, \mu j, \tau j \text { unchanged }\end{array}$ \\
\hline $\begin{array}{l}\Psi_{1} \Psi_{2}^{*}\left(\Psi_{1}^{*} \Psi_{2}\right) \\
\Phi_{1}=\Phi_{2}=0\end{array}$ & $\begin{array}{c}\mu^{+} j j \bar{\nu}: \\
(40-60 \%) \times 100 \% \\
\end{array}$ & $\begin{array}{ll}e^{+} b b \bar{\nu}: & (40-50 \%) \times 50 \% \\
\mu^{+} j b \bar{\nu}: & (20-30 \%) \times 50 \%\end{array}$ & $\begin{array}{cc}\mu^{+} s j \bar{\nu}, e^{+} b j \bar{\nu} & 2(30 \%)^{2} \\
\mu^{+} s b \bar{\nu}, e^{+} b b \bar{\nu} & (30 \%)^{2} \\
\end{array}$ \\
\hline$\Phi_{1} \approx \pi, \Phi_{2}=0$ & independent of $\Phi_{1}$ & $e b \leftrightarrow e j, \mu j \leftrightarrow \mu b$ & $e b \leftrightarrow e j, \mu s \leftrightarrow \mu b$ \\
\hline$\Phi_{1}=0, \Phi_{2} \approx \pi$ & $\mu^{ \pm} j j \bar{\nu}$ unchanged & independent of $\Phi_{2}$ & $e b, \mu s$ unchanged \\
\hline
\end{tabular}

TABLE V: Leading fully reconstructible channels and the indicative ranges of their branching fractions. The light neutrino mass patterns of the $\mathrm{NH}$ and IH with the lightest neutrino mass $m_{0} \lesssim 10^{-2} \mathrm{eV}$ are shown in the first two columns, including the effects of vanishing and large Majorana phases. The last column shows the Quasi degenerate neutrino mass pattern when $m_{0}>0.1 \mathrm{eV}$. A jet of a light quark $(d, s)$ is denoted by $j$.

- $\Psi_{2}$ decays are independent of any phases. They are thus robust to test the mass pattern.

- $\Psi_{1}$ decays to a lepton plus inclusive jets are also independent of any phases. They are robust to test the mass pattern as well.

- These channels are not sensitive to Majorana phase $\Phi_{2}$.

- The sensitivity to $\Phi_{1}$ can be significant if $b$-flavor tagging can be exploited in the cases of the IH and QD.

For illustration, consider first the cleanest channel, $\Psi_{1} \Psi_{1}^{*} \rightarrow \mu^{+} j \mu^{-} j(j=d, s)$. The number of events is written as

$$
N=L \times \sigma\left(p p \rightarrow \Psi_{1} \Psi_{1}^{*}\right) \times \mathrm{BR}^{2}\left(\Psi_{1} \rightarrow \mu^{+} j\right)
$$



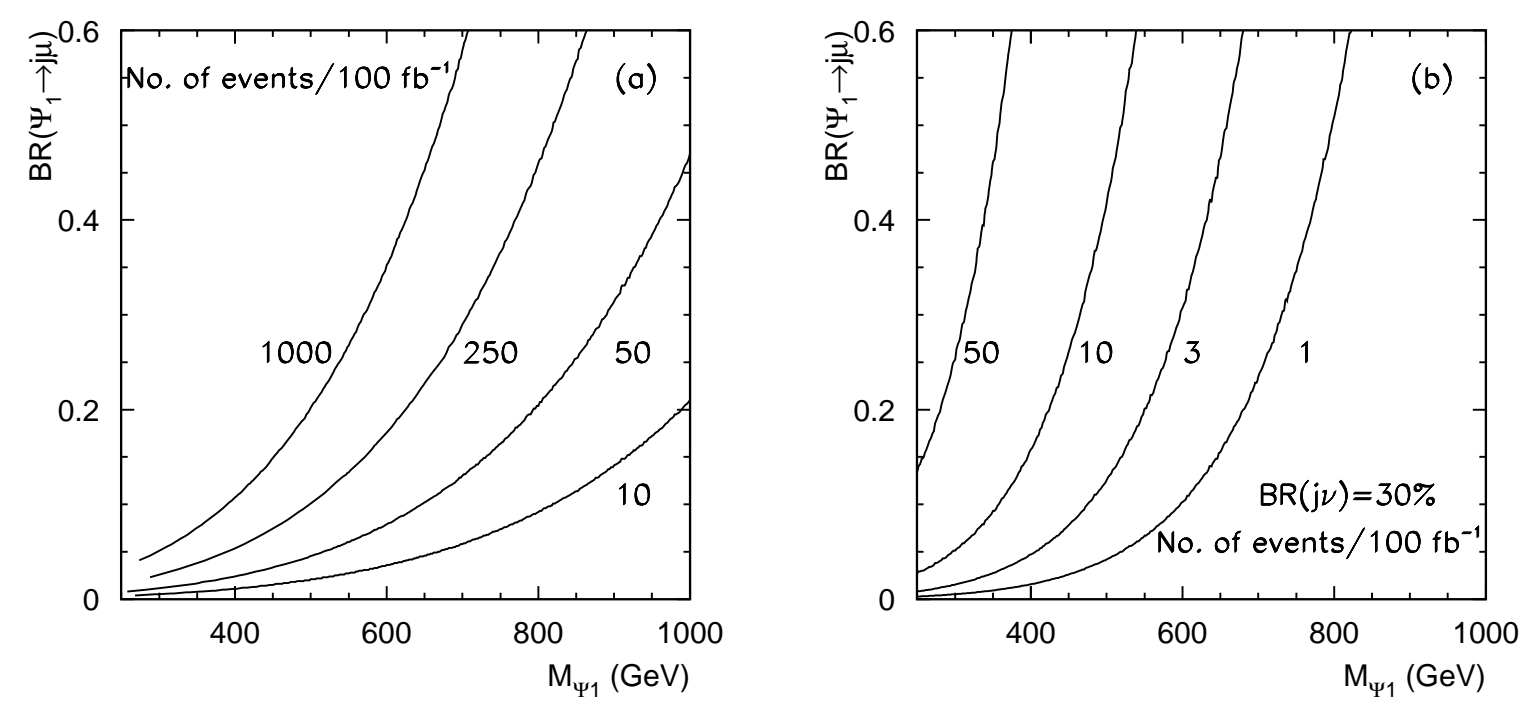

FIG. 27: Event contours in the BR $-M_{\Psi_{1}}$ plane at the LHC with an integrated luminosity $100 \mathrm{fb}^{-1}$ for (a) $\Psi_{1} \Psi_{1}^{*} \rightarrow$ $\mu^{+} j \mu^{-} j$, and (b) $\Psi_{1} \Psi_{2}^{*}+\Psi_{1}^{*} \Psi_{2} \rightarrow \mu^{+} j j \nu$, including all the judicious cuts describe in the early sections.

where $L$ is the integrated luminosity. Given a sufficient number of events $N$, the mass of leptoquark is determined by the invariant mass of lepton and jet $M_{\ell j}$. We thus predict the corresponding production rate $\sigma\left(p p \rightarrow \Psi_{1} \Psi_{1}^{*}\right)$ for this given mass. The only unknown in the Eq. (79) is the decay branching fraction. We present the event contours in the $\mathrm{BR}-M_{\Psi_{1}}$ plane in Fig. 27(a) for $100 \mathrm{fb}^{-1}$ luminosity including all the judicious cuts described earlier, with which the backgrounds are insignificant. We see that the LHC has tremendous sensitivity to probe the channel $\Psi_{1} \rightarrow \mu+$ jet within and beyond the predicted branching fraction (35\% - 60\% in NH, $20 \%-30 \%$ in $\mathrm{IH}$, and $30 \%$ in QD) up to and beyond $M_{\Psi_{1}} \sim 1 \mathrm{TeV}$.

Since the $\Psi_{2}$ decay is independent of the unknown Majorana phases, it is thus important to search for the $\Psi_{1} \Psi_{2}^{*}\left(\Psi_{1}^{*} \Psi_{2}\right)$ signal. In Fig. 27 b), we show the event contours in the BR $-M_{\Psi_{1}}$ plane, for $100 \mathrm{fb}^{-1}$ luminosity including all the judicious cuts described earlier. We see that with the estimated branching fraction for $\nu+$ jet, one can reach the coverage of about $M_{\Psi_{1}} \sim 0.6 \mathrm{TeV}$ or higher.

\section{Single Production of $\Psi_{1}$}

The leptoquark pair production is well predicted by its $\mathrm{SU}(3)_{c}$ and $\mathrm{SU}(2)_{L}$ gauge interactions, and their decays studied in the previous sections are governed by the neutrino oscillation parameters, and all are model-independent. The drawback for this nice feature, however, is that it does not provide the modeldependent details, such as the Yukawa couplings or equivalently the vev $v_{\Delta}$. In order to further test the 

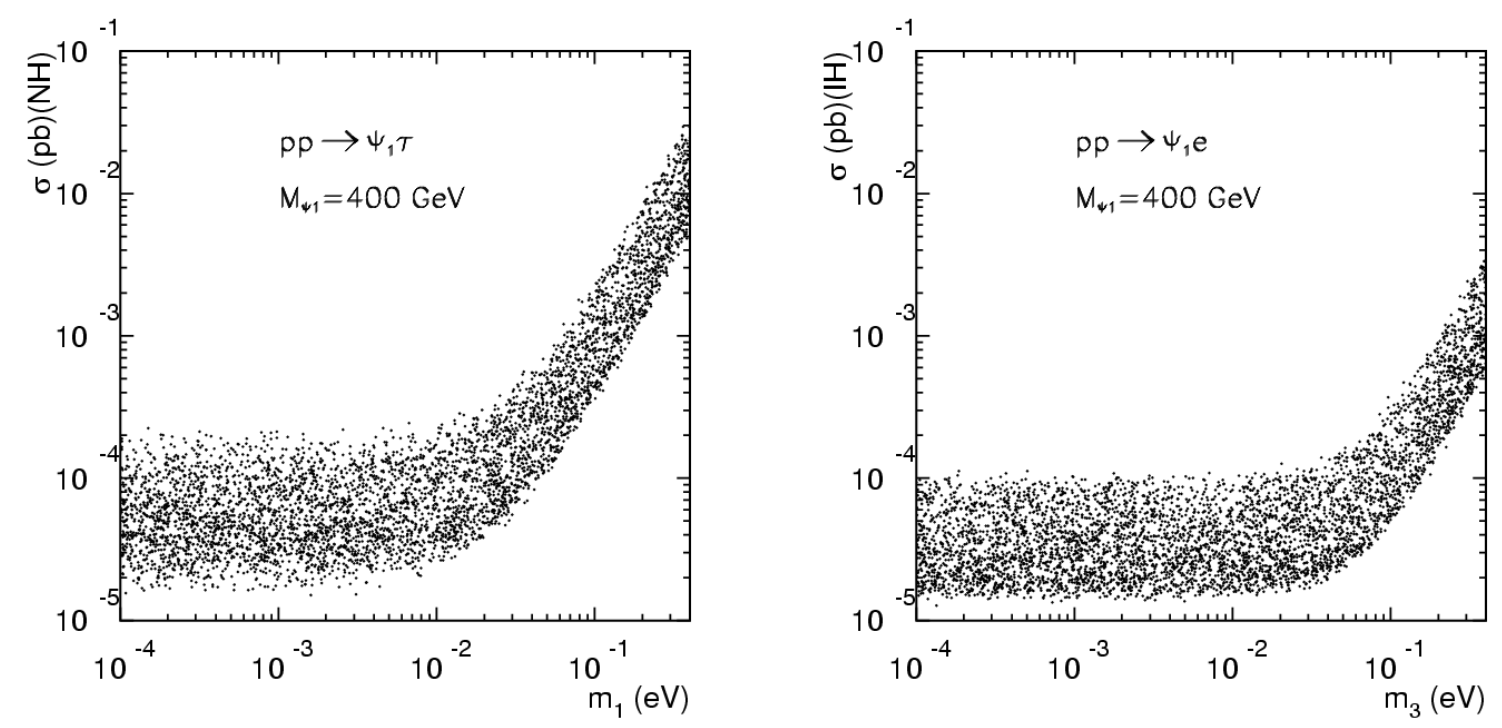

FIG. 28: Total cross section for single $\Psi_{1}$ production with $4 \mathrm{eV} \leq v_{\Delta} \leq 10 \mathrm{eV}$.

theory, one needs to involve the Yukawa couplings in the production process. This is the single production of a leptoquark, as in Eq. (55).

The parton level cross section for this process is

$$
\frac{d \sigma}{d \hat{t}}\left(g d_{i}\left(\bar{d}_{i}\right) \rightarrow \Psi_{1}\left(\Psi_{1}^{*}\right) \ell_{j}^{-}\left(\ell_{j}^{+}\right)\right)=\frac{\left|\Gamma_{1}^{i j}\right|^{2} \alpha_{s}}{48 \hat{s}^{2}}\left[\frac{\hat{s}+\hat{t}-M_{\Psi_{1}}^{2}}{\hat{s}}+\frac{\hat{t}\left(\hat{t}+M_{\Psi_{1}}^{2}\right)}{\left(\hat{t}-M_{\Psi_{1}}^{2}\right)^{2}}+\frac{\hat{t}\left(2 M_{\Psi_{1}}^{2}-\hat{s}\right)}{\hat{s}\left(\hat{t}-M_{\Psi_{1}}^{2}\right)}\right]
$$

where $\hat{t}=\left(p_{d_{i}}-p_{\ell_{j}}\right)^{2}$. Taking into account the neutrino oscillation constraints, in Fig. 28 we show the scatter plots for the total cross section of single $\Psi_{1}$ production versus the lightest neutrino mass, for the $\mathrm{NH}$ case (left) and the IH case (right). The total cross sections for both cases are less than $1 \mathrm{fb}$, although it can become larger when reaching the quasi degenerate case for $m_{\nu}>0.1 \mathrm{eV}$. To estimate the backgrounds, we start from the cleanest channels with $e, \mu$ in the final state from $\Psi_{1}$ decay. The signal consists of one pair of opposite-sign same-flavor leptons and one light jet. We employ the same basic acceptance cuts and smearing parameters as the pair production.

The irreducible SM backgrounds to this channel are

$$
Z^{*} / \gamma^{*} j \rightarrow \ell^{+} \ell^{-} j, \quad W^{+} W^{-} j \rightarrow \ell^{+} \ell^{-} j+E_{T} .
$$

Once again, the signal and background kinematics are quite. We outline the characteristics and propose some judicious cuts as follows.

- The leptons and jets from the leptoquark decay are very hard. We tighten up the $p_{T}$ cuts

$$
p_{T}^{\max }(\ell)>M_{\Psi_{1}} / 4, \quad p_{T}^{\max }(j)>M_{\Psi_{1}} / 4 \text {. }
$$




\begin{tabular}{|c||c|c|c|c|c|c|}
\hline $\begin{array}{c}\sigma(\mathrm{pb}) \\
\text { cuts }\end{array}$ & $\begin{array}{c}\text { Basic } \\
\text { Cuts }\end{array}$ & $\begin{array}{c}\cos \phi_{\ell \ell} \\
>-0.5\end{array}$ & $\begin{array}{c}p_{T}^{\ell}, p_{T}^{j} \text { cut } \\
>100,100 \mathrm{GeV}\end{array}$ & $\begin{array}{c}M_{Z} \text { veto } \\
\pm 15 \mathrm{GeV}\end{array}$ & $\begin{array}{c}M_{W} \text { veto } \\
E_{T}<25 \mathrm{GeV}\end{array}$ & $\begin{array}{c}M_{\ell j} \\
\pm 20 \mathrm{GeV}\end{array}$ \\
\hline$j \gamma^{*} / Z^{*}$ & 309 & 113 & 9.3 & 0.82 & 0.82 & $6.5 \times 10^{-2}$ \\
\hline$j W^{ \pm} W^{\mp}$ & 0.87 & 0.48 & $5.8 \times 10^{-2}$ & $4.7 \times 10^{-2}$ & $5.6 \times 10^{-3}$ & $4.3 \times 10^{-4}$ \\
\hline
\end{tabular}

TABLE VI: Backgrounds for a $400 \mathrm{GeV}$ leptoquark single production.

- Because the two leptons are largely back-to-back for $j Z^{*} / \gamma^{*}$ background, we can set a cut for the angle between the two leptons in the transverse plane $\cos \phi_{\ell \ell}>-0.5$.

- To remove the $Z$ background, we veto the lepton pairs with opposite charges in the $Z$-mass window $\left|M_{\ell^{+} \ell^{-}}-M_{Z}\right|>15 \mathrm{GeV}$.

- To remove the $j W W$ background, we veto the missing energy from $W$ decay and require $E_{T}<$ $25 \mathrm{GeV}$.

- In order to select the correct lepton and jet combination and reconstruct the leptoquark, we make use of the feature that the lepton from leptoquark decay is harder than that produced with $\Psi_{1}$, as seen in Fig. 29(a).

- Finally, when we perform a signal significance analysis, we look for a resonance in the mass distribution of $\ell j$. The invariant mass of $\ell+j$ is plotted in Fig. 29 b) with the event selection for $400 \mathrm{GeV}$ leptoquark mass. The long tail in the high mass region is due to the wrong choice of the lepton. We propose to look at a mass window of $\left|M_{\ell j}-M_{\Psi_{1}}\right|<M_{\Psi_{1}} / 20$.

We summarize the background rates with the above consecutive cuts, optimized for a $M_{\Psi_{1}}=400 \mathrm{GeV}$ signal in Table. VI Unfortunately, even after all these optimal cuts, the $\gamma^{*} / Z j$ background is still larger than the signal by more than an order of magnitude for both the NH and IH cases. Unless we are deep in the quasi degenerate situation when the Yukawa coupling is substantially larger, the single LQ production would not be observable above the huge SM background. We will not pursue this channel further.

\section{SUMMARY AND CONCLUSIONS}

We have studied the properties of light scalar leptoquarks, $\Phi_{b}^{T}=\left(\Psi_{1}, \Psi_{2}\right) \sim(3,2,1 / 6)$, in the context of a simple grand unified theory where the neutrino masses are generated through the Type II seesaw mechanism. The $S U(5)$ symmetry of this theory implies that the coupling of the leptoquarks to matter are governed by the neutrino mass matrix. We considered the low energy constraints on the model parameters 

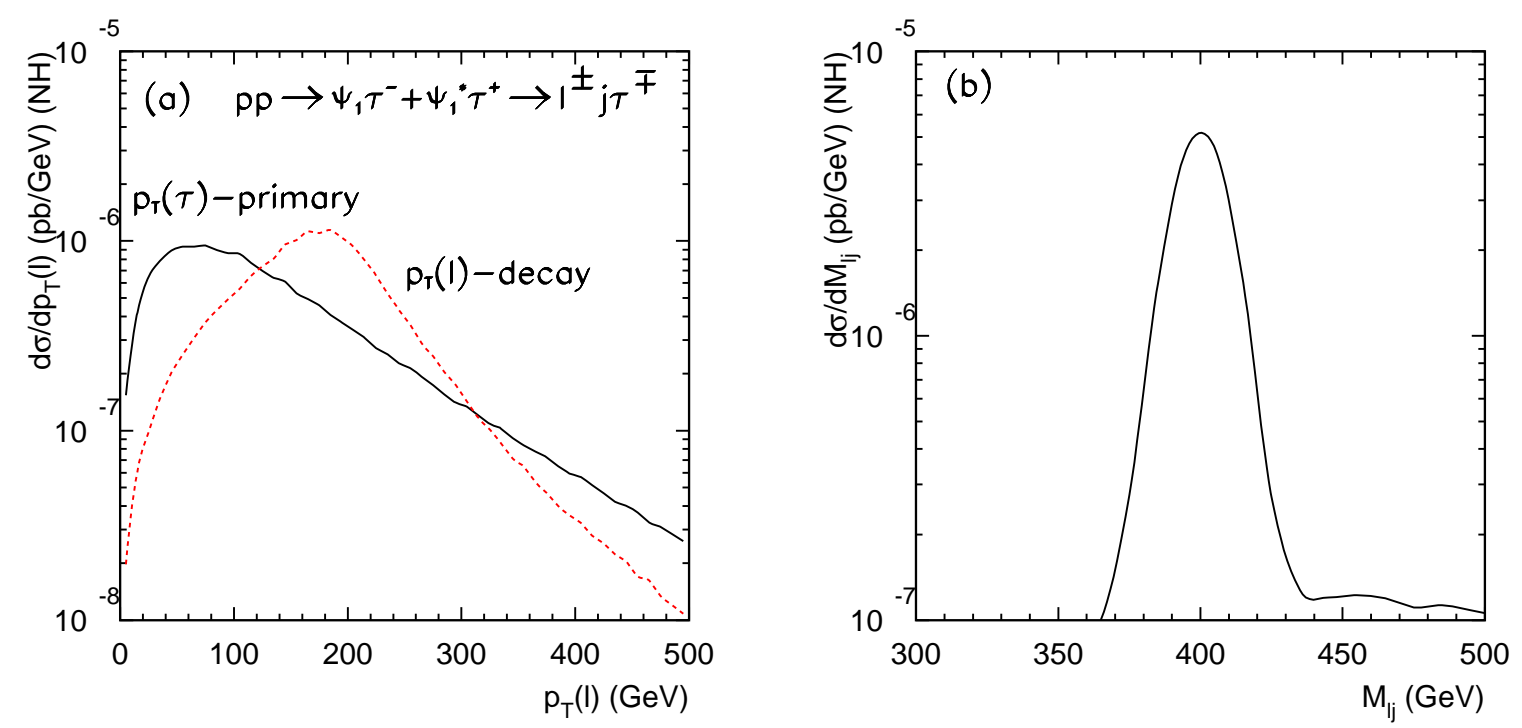

FIG. 29: (a) $p_{T}(\ell)$ distributions for the primary lepton and that from $\Psi_{1}$ decay, and (b) invariant mass $M(\ell j)$ for a $400 \mathrm{GeV}$ single leptoquark production.

and explored the feasibility of testing the theory at the LHC with detailed parton-level simulations for the signal and SM backgrounds. We focus mainly on the different scenarios where the semileptonic decays of leptoquarks are the dominant decay channels. We found the following interesting results:

- Among the low-energy constraints coming from meson decays, lepton flavour violation, and electroweak precision data, the strongest bound is from the $K_{L} \rightarrow \mu^{+} \mu^{-}$decay, that leads to $M_{\Psi_{1}} v_{\Delta}>$ $1600 \mathrm{GeV} \cdot \mathrm{eV}$.

- The decays of the scalar leptoquarks are quite different in each spectrum for neutrino masses. Consider the most important $\Psi_{1}$ decay. In the NH scenario, the leading decay channels are $\mu+j$ and $\tau+j$, while the $e+j$ channel is absent. In the IH scenario, the $e+b$ channel is the leading one, while $\mu+j$ and $\tau+j$ are next. In the Quasi-degenerate case, the leading channels are shared by $e b \approx \mu s \approx \tau d$.

- The decays of $\Psi_{2}$ are independent of the unknown phases in the theory. One can use these decays to test the neutrino mass pattern without additional ambiguity.

- The signal dependence on the Majorana phase $\Phi_{2}$ is very weak. However, the dependence on $\Phi_{1}$ in the case of IH and QD hierarchy can be strong, and determining the $b \ell$ contributions could help probe 
$\Phi_{1}$ if we can effectively exploit $b$-flavor tagging. Specifically, we have, for the IH or QD hierarchy,

$$
\operatorname{BR}(b e) \begin{cases}\gg \mathrm{BR}(b \mu), \mathrm{BR}(b \tau) & \text { for } \Phi_{1} \approx 0 \\ \ll \mathrm{BR}(b \mu), \mathrm{BR}(b \tau) & \text { for } \Phi_{1} \approx \pi\end{cases}
$$

We reiterate that due to the symmetry in the neutrino mass matrix, the theory has the interesting prediction for the correlations $\operatorname{BR}(b \mu)=\operatorname{BR}(s e)$ and $\operatorname{BR}(b \tau)=\operatorname{BR}(d e)$. We also note that the difference between the IH and QD cases is rather small, with $\ell b$ branching fraction to be $30 \%$ in the QD case, compared to $40-50 \%$ in the IH case.

- The leading production of the leptoquarks is via the QCD process, $p p \rightarrow \Psi_{1} \Psi_{1}^{*}$. We demonstrated that besides the clean di-lepton channels from $e, \mu$, the $\tau$ final state can be effectively reconstructed as well. Even with only the clean channel $\mu^{+} \mu^{-}+$jets, the signal observability can extend to $M_{\Psi_{1}} \sim 1$ $\mathrm{TeV}$ and beyond.

- Although the rate is smaller than for QCD pair production, the electroweak process of associated production $\Psi_{1} \Psi_{2}^{*}\left(\Psi_{1}^{*} \Psi_{2}\right)$ can be very useful by making use of both $\Psi_{1}, \Psi_{2}$ decays simultaneously to identify the quantum numbers of the leptoquarks and to distinguish between the neutrino mass spectra. Even with only the clean channel of $\mu+E_{T}+$ jets, the signal observability can reach about $M_{\Psi_{1}} \sim 0.6 \mathrm{TeV}$ or higher.

- We have found that the single leptoquark production via its Yukawa couplings is very small in both $\mathrm{NH}$ and $\mathrm{IH}$ spectrum.

The discovery of these leptoquarks at the LHC could test this appealing scenario for neutrino mass generation at a fundamental level, and give us a hint about a possible candidate theory for grand unification.

\section{Acknowledgments}

The work of P. F. P. and M.R-M. was supported in part by the U.S. Department of Energy contract No. DE-FG02-08ER41531 and in part by the Wisconsin Alumni Research Foundation. P. F. P would like to thank I. Dorsner, R. Gonzalez Felipe, G. Rodrigo and G. Senjanović for discussions. The work of T. H. is supported in part by the U.S. Department of Energy under grant No. DE-FG02-95ER40896, and by the Wisconsin Alumni Research Foundation. T. L. would like to thank Kai Wang for discussions and the Ministry of Education of China for support and would also like to acknowledge the hospitality of the Phenomenology Institute, University of Wisconsin-Madison while the work was carried out. 


\section{APPENDIX A: EXPLICIT EXPRESSIONS FOR $\Gamma_{1}$ AND $\Gamma_{2}$}

As seen from Eq. (9), we have

$$
\Gamma_{1}=\Gamma_{2} V_{P M N S}^{\dagger} K_{3}^{*} \text { and } \Gamma_{2}=B K_{3}^{*} V_{P M N S}^{*} \frac{m_{\nu}^{\text {diag }}}{v_{\Delta}}
$$

and define

$$
\begin{gathered}
Y_{1}^{i j}=\Gamma_{1}^{i j} \times v_{\Delta}, \quad Y_{2}^{i}=\sum_{j=1}^{3}\left|\Gamma_{2}^{i j}\right|^{2} \times v_{\Delta}^{2}, \\
B=\left(\begin{array}{ccc}
0 & 0 & e^{i \beta_{1}} \\
0 & e^{i \beta_{2}} & 0 \\
e^{i \beta_{3}} & 0 & 0
\end{array}\right), K_{3}=\left(\begin{array}{lll}
e^{i \alpha_{1}} & 0 & 0 \\
0 & e^{i \alpha_{2}} & 0 \\
0 & 0 & e^{i \alpha_{3}}
\end{array}\right) \\
Y_{2}^{1}=c_{13}^{2} c_{23}^{2} m_{3}^{2}+c_{12}^{2} c_{23}^{2} s_{13}^{2} m_{1}^{2}+c_{23}^{2} s_{12}^{2} s_{13}^{2} m_{2}^{2}+c_{12}^{2} s_{23}^{2} m_{2}^{2}+s_{12}^{2} s_{23}^{2} m_{1}^{2} \\
-2 c_{12} c_{23} s_{12} s_{13} s_{23} \cos (\delta)\left(m_{1}^{2}-m_{2}^{2}\right), \\
Y_{2}^{2}=c_{23}^{2} s_{12}^{2} m_{1}^{2}+c_{13}^{2} s_{23}^{2} m_{3}^{2}+s_{12}^{2} s_{13}^{2} s_{23}^{2} m_{2}^{2}+c_{12}^{2}\left(c_{23}^{2} m_{2}^{2}+s_{13}^{2} s_{23}^{2} m_{1}^{2}\right) \\
+2 c_{12} c_{23} s_{12} s_{13} s_{23} \cos (\delta)\left(m_{1}^{2}-m_{2}^{2}\right), \\
Y_{2}^{3}=c_{12}^{2} c_{13}^{2} m_{1}^{2}+c_{13}^{2} s_{12}^{2} m_{2}^{2}+s_{13}^{2} m_{3}^{2} .
\end{gathered}
$$

and

$$
\begin{gathered}
e^{-i \beta_{3}} Y_{1}^{31}=e^{-2 i \alpha_{1}}\left(c_{13}^{2} s_{12}^{2} m_{2}+c_{12}^{2} c_{13}^{2} e^{-i \Phi_{1}} m_{1}+s_{13}^{2} e^{2 i \delta} e^{-i \Phi_{2}} m_{3}\right) \\
e^{-i \beta_{2}} Y_{1}^{21}=e^{-i \beta_{3}} Y_{1}^{32}=e^{-i\left(\alpha_{1}+\alpha_{2}\right)} c_{13}\left(s_{12}\left(c_{12} c_{23}-s_{12} s_{13} s_{23} e^{-i \delta}\right) m_{2}\right. \\
\left.+c_{12}\left(-c_{23} s_{12}-c_{12} s_{13} s_{23} e^{-i \delta}\right) e^{-i \Phi_{1}} m_{1}+s_{13} s_{23} e^{i \delta} e^{-i \Phi_{2}} m_{3}\right) \\
e^{-i \beta_{1}} Y_{1}^{11}=e^{-i \beta_{3}} Y_{1}^{33}=e^{-i\left(\alpha_{1}+\alpha_{3}\right)} c_{13}\left(s_{12}\left(-c_{12} s_{23}-s_{12} s_{13} c_{23} e^{-i \delta}\right) m_{2}\right. \\
\left.+c_{12}\left(s_{23} s_{12}-c_{12} s_{13} c_{23} e^{-i \delta}\right) e^{-i \Phi_{1}} m_{1}+s_{13} c_{23} e^{i \delta} e^{-i \Phi_{2}} m_{3}\right) \\
e^{-i \beta_{2}} Y_{1}^{22}=e^{-2 i \alpha_{2}}\left(\left(c_{12} c_{23}-s_{12} s_{13} s_{23} e^{-i \delta}\right)^{2} m_{2}+\left(c_{23} s_{12}+c_{12} s_{13} s_{23} e^{-i \delta}\right)^{2} e^{-i \Phi_{1}} m_{1}\right. \\
\left.+c_{13}^{2} s_{23}^{2} e^{-i \Phi_{2}} m_{3}\right), \\
e^{-i \beta_{1}} Y_{1}^{12}=e^{-i \beta_{2}} Y_{1}^{23}=e^{-i\left(\alpha_{2}+\alpha_{3}\right)}\left(-\left(c_{12} s_{23}+c_{23} s_{12} s_{13} e^{-i \delta}\right)\left(c_{12} c_{23}-s_{12} s_{13} s_{23} e^{-i \delta}\right) m_{2}\right. \\
+\left(s_{12} s_{23}-c_{12} c_{23} s_{13} e^{-i \delta}\right)\left(-c_{23} s_{12}-c_{12} s_{13} s_{23} e^{-i \delta}\right) e^{-i \Phi_{1}} m_{1} \\
\left.+c_{13}^{2} c_{23} s_{23} e^{-i \Phi_{2}} m_{3}\right), \\
e^{-i \beta_{1}} Y_{1}^{13}=e^{-2 i \alpha_{3}}\left(\left(c_{12} s_{23}+c_{23} s_{12} s_{13} e^{-i \delta}\right)^{2} m_{2}+\left(s_{12} s_{23}-c_{12} c_{23} s_{13} e^{-i \delta}\right)^{2} e^{-i \Phi_{1}} m_{1}\right. \\
\left.+c_{13}^{2} c_{23}^{2} e^{-i \Phi_{2}} m_{3}\right) .
\end{gathered}
$$




\section{APPENDIX B: THE CONTRIBUTIONS OF LEPTOQUARKS TO THE OBLIQUE PARAMETERS}

We have computed the renormalized self-energy functions $\hat{\Pi}_{V V^{\prime}}\left(q^{2}\right)$ in the $\overline{\mathrm{MS}}$ scheme and find that $\hat{\Pi}_{V V^{\prime}}(0)=0$ for $V, V^{\prime}=Z, \gamma$. Thus, the $T$ parameter is determined entirely by $\hat{\Pi}_{W W}(0)$ and reads

$$
\hat{\alpha}\left(M_{Z}\right) T=\frac{\hat{\alpha}}{8 \pi \hat{s}^{2}} \frac{N_{C}}{M_{W}^{2}}\left[\frac{1}{2}\left(M_{1}^{2}+M_{2}^{2}\right)+\frac{M_{1}^{2} M_{2}^{2}}{M_{1}^{2}-M_{2}^{2}} \ln \frac{M_{2}^{2}}{M_{1}^{2}}\right],
$$

where $M_{k} \equiv M_{\Psi_{k}}(k=1,2)$ and where the running parameters $\hat{\alpha}$ and $\hat{s}$ on the RHS have been evaluated at the scale $\mu=M_{Z}$. The $S$ parameter, in contrast, requires inclusion of $\hat{\Pi}_{V V^{\prime}}\left(M_{Z}^{2}\right) \neq 0$ for $V, V^{\prime}=Z, \gamma$, leading to

$$
\begin{aligned}
\hat{\alpha}\left(M_{Z}\right) S= & \frac{\hat{\alpha} N_{C} Y_{\mathrm{LQ}}}{\pi M_{Z}^{2}} \sum_{k=1}^{2}\left[M_{Z}^{2} F\left(M_{k}^{2}, M_{k}^{2}, M_{Z}^{2}\right)-\frac{M_{Z}^{2}}{6} \ln M_{Z}^{2}\right. \\
& \left.+M_{k}^{2} \ln M_{k}^{2}-2 M_{k}^{2} F_{1}\left(M_{k}^{2}, M_{k}^{2}, M_{Z}^{2}\right)\right],
\end{aligned}
$$

where the sum runs over the two LQ states having hypercharge $Y_{\mathrm{LQ}}$ and where

$$
\begin{aligned}
F_{n}(a, b, c) & =\int_{0}^{1} d x x^{n} \ln [a(1-x)+b x-c x(1-x)-i \varepsilon] \\
F & =F_{1}-F_{2}
\end{aligned}
$$

[1] For a review on GUTs, see for example G. Senjanović, "Course on grand unification," Prepared for 2nd International Summer School in High Energy Physics, Mugla, Turkey, 25-30 Sep 2006.

[2] For a review see P. Nath and P. Fileviez Pérez, "Proton stability in grand unified theories, in strings, and in branes," Phys. Rept. 441 (2007) 191 [arXiv:hep-ph/0601023]. For future proton decay experiments see D. Autiero et al., "Large underground, liquid based detectors for astro-particle physics in Europe: scientific case and prospects," JCAP 0711 (2007) 011 [arXiv:0705.0116 [hep-ph]].

[3] S. Rolli, M. Tanabashi in PDG, "Leptoquarks," W.-M. Yao et al., J. Phys. G 33, 1 (2006).

[4] W. Buchmuller, R. Ruckl and D. Wyler, "Leptoquarks in lepton quark collisions," Phys. Lett. B 191 (1987) 442 [Erratum-ibid. B 448 (1999) 320].

[5] J. L. Hewett and T. G. Rizzo, "Low-Energy Phenomenology of Superstring Inspired E(6) Models," Phys. Rept. 183 (1989) 193.

[6] I. Dorsner and P. Fileviez Pérez, "Unification without supersymmetry: Neutrino mass, proton decay and light leptoquarks,” Nucl. Phys. B 723 (2005) 53 [arXiv:hep-ph/0504276].

[7] W. Konetschny and W. Kummer, "Nonconservation Of Total Lepton Number With Scalar Bosons," Phys. Lett. B 70 (1977) 433; T. P. Cheng and L. F. Li, "Neutrino Masses, Mixings And Oscillations In SU(2) X U(1) Models Of Electroweak Interactions," Phys. Rev. D 22 (1980) 2860; G. Lazarides, Q. Shafi and C. Wetterich, 
“Proton Lifetime And Fermion Masses In An SO(10) Model,” Nucl. Phys. B 181 (1981) 287; J. Schechter and J. W. F. Valle, “Neutrino Masses In SU(2) X U(1) Theories,” Phys. Rev. D 22 (1980) 2227; R. N. Mohapatra and G. Senjanović, “Neutrino Masses And Mixings In Gauge Models with Spontaneous Parity Violation,” Phys. Rev. D 23 (1981) 165.

[8] I. Dorsner, P. Fileviez Pérez and R. Gonzalez Felipe, "Phenomenological and cosmological aspects of a minimal GUT scenario,” Nucl. Phys. B 747 (2006) 312 [arXiv:hep-ph/0512068].

[9] I. Dorsner, P. Fileviez Pérez and G. Rodrigo, "Fermion Masses and the UV Cutoff of the Minimal Realistic SU(5)," Phys. Rev. D 75 (2007) 125007 [arXiv:hep-ph/0607208].

[10] H. Georgi and S. L. Glashow, “Unity Of All Elementary Particle Forces,” Phys. Rev. Lett. 32 (1974) 438.

[11] M. Cirelli, N. Fornengo and A. Strumia, "Minimal dark matter," Nucl. Phys. B 753, 178 (2006) [arXiv:hep-ph/0512090].

[12] I. Dorsner and P. Fileviez Pérez, "How long could we live?," Phys. Lett. B 625 (2005) 88 [arXiv:hep-ph/0410198].

[13] P. Fileviez Pérez, "Upper bound on the proton lifetime and the minimal non-SUSY grand unified theory," AIP Conf. Proc. 903 (2006) 385 [arXiv:hep-ph/0606279].

[14] T. Schwetz, "Neutrino oscillations: present status and outlook," arXiv:0710.5027 [hep-ph]; M. Maltoni, T. Schwetz, M. A. Tortola and J. W. F. Valle, "Status of global fits to neutrino oscillations," New J. Phys. 6 (2004) 122 [arXiv:hep-ph/0405172]. See also: M. C. Gonzalez-Garcia and M. Maltoni, "Phenomenology with Massive Neutrinos," Phys. Rept. 460 (2008) 1 [arXiv:0704.1800[hep-ph]]; A. Strumia and F. Vissani, “Neutrino masses and mixings and., arXiv:hep-ph/0606054.

[15] P. Fileviez Perez, T. Han, G. Y. Huang, T. Li and K. Wang, "Neutrino Masses and the LHC: Testing Type II Seesaw,’ Phys. Rev. D 78 (2008) 015018 [arXiv:0805.3536 [hep-ph]]; Phys. Rev. D 78 (2008) 071301 [arXiv:0803.3450 [hep-ph]].

[16] W. M. Yao et al. [Particle Data Group], "Review of particle physics,” J. Phys. G33 (2006) 1.

[17] S. Davidson, D. Bailey and B. A. Campbell, "Model independent constraints on leptoquarks from rare processes,” Z. Phys. C61 (1994) 613 [arXiv:hep-ph/9309310].

[18] M. Leurer, “A Comprehensive Study of Leptoquark Bounds,” Phys. Rev. D49 (1994) 333 arXiv:hep-ph/9309266.

[19] D. G. Dumm and A. Pich, "Long-distance contributions to the $K_{L} \rightarrow \mu^{+} \mu^{-}$decay width," Phys. Rev. Lett. 80 (1998) 4633 [arXiv:hep-ph/9801298].

[20] D. Gomez Dumm and A. Pich, "Short- and long-distance contributions to the rare decay K(L) to mu+ mu-," Nucl. Phys. Proc. Suppl. 74, 186 (1999) [arXiv:hep-ph/9810523].

[21] W. Bertl et al. [SINDRUM II Collaboration], “A Search for $\mu-e$ conversion in muonic gold,” Eur. Phys. J. C 47, 337 (2006).

[22] R. Kitano, M. Koike and Y. Okada, "Detailed calculation of lepton flavor violating muon-electron conversion rate for various nuclei,” Phys. Rev. D66 (2002) 096002, Erratum-ibid. D76 (2007) 059902 [arXiv:hep-ph/0203110]

[23] M. E. Peskin and T. Takeuchi, "A New constraint on a strongly interacting Higgs sector," Phys. Rev. Lett. 65, 
964 (1990).

[24] J. F. Gunion, R. Vega and J. Wudka, "Naturalness Problems For Rho=1 And Other Large One Loop Effects For A Standard Model Higgs Sector Containing Triplet Fields,” Phys. Rev. D43 (1991) 2322.

[25] B. Dion, L. Marleau and G. Simon, "Scalar Leptoquark Pair Production at the CERN LHC: Signal and Backgrounds," Eur. Phys. J. C2 (1998) 497 [arXiv:hep-ph/9701285].

[26] M. Krämer, T. Plehn, M. Spira and P. M. Zerwas, "Pair production of scalar leptoquarks at the CERN LHC," Phys. Rev. D 71 (2005) 057503 arXiv:hep-ph/0411038.

[27] J. L. Hewett and S. Pakvasa, "Scalar-leptoquark production at hadron colliders," Phys. Rev. D 37 (1988) 3165; O. J. P. Eboli, R. Zukanovich Funchal and T. L. Lungov, "Signal and backgrounds for leptoquarks at the CERN LHC,” Phys. Rev. D 57, 1715 (1998) arXiv:hep-ph/9709319].

[28] A. Belyaev, C. Leroy, R. Mehdiyev and A. Pukhov, "Leptoquark single and pair production at LHC with CalcHEP/CompHEP in the complete model," JHEP 0509:005 (2005) [arXiv:hep-ph/0502067].

[29] A. A. Affolder et al. [CDF Collaboration], "Search for second and third generation leptoquarks including production via technicolor interactions in $p \bar{p}$ collisions at $\sqrt{s}=1.8$ TeV,” Phys. Rev. Lett. 85, 2056 (2000) [arXiv:hep-ex/0004003]; D. E. Acosta et al. [CDF Collaboration], "Search for first-generation scalar leptoquarks in $p \bar{p}$ collisions at $\sqrt{s}=1.96$ TeV," Phys. Rev. D 72, 051107 (2005) [arXiv:hep-ex/0506074]; A. Abulencia et al. [CDF Collaboration], "Search for second-generation scalar leptoquarks in $p \bar{p}$ collisions at $\sqrt{s}=1.96-\mathrm{TeV}$," Phys. Rev. D 73, 051102 (2006) [arXiv:hep-ex/0512055].

[30] V. M. Abazov et al. [D0 Collaboration], "Search for first-generation scalar leptoquarks in p anti-p collisions at $\sqrt{s}=1.96$ TeV," Phys. Rev. D 71, 071104 (2005), arXiv:hep-ex/0412029]; D0 Collaboration, "Searches for Leptoquark Production at D0," arXiv:0710.0255 [hep-ex]]; D0 Collaboration, V. M. Abazov et al., "Search for third generation scalar leptoquarks decaying into $\tau b$," [arXiv:0806.3527 [hep-ex]]; "Search for pair production of second generation scalar leptoquarks," arXiv:0808.4023 [hep-ex].

[31] CMS TDR: CMS Physics: Technical Design Report V.2: Physics Performance, CERN-LHCC-2006-021; S. Abdullin and F. Charles, "Study of leptoquark pair production at the LHC with the CMS detector," Phys. Lett. B 464, 223 (1999) [arXiv:hep-ph/9905396.

[32] F. Maltoni and T. Stelzer, "MadEvent: Automatic event generation with MadGraph," JHEP 0302:027 (2003) [arXiv:hep-ph/0208156]. 